\title{
Chiral Structure of Scalar and Pseudoscalar Mesons
}

\author{
Hua-Xing Chen \\ School of Physics and Nuclear Energy Engineering and International Research Center for Nuclei and Particles in the Comos, \\ Beihang University, Beijing 100191, China
}

Correspondence should be addressed to Hua-Xing Chen; hxchen@buaa.edu.cn

Received 12 July 2013; Accepted 4 November 2013

Academic Editor: Shi-Hai Dong

Copyright (C) 2013 Hua-Xing Chen. This is an open access article distributed under the Creative Commons Attribution License, which permits unrestricted use, distribution, and reproduction in any medium, provided the original work is properly cited.

We systematically study the chiral structure of local tetraquark currents of flavor singlet and $J^{P}=0^{+}$. We also investigate their chiral partners, including scalar and pseudoscalar tetraquark currents of flavor singlet, octet, $\mathbf{1 0}, \overline{\mathbf{1 0}}$, and $\mathbf{2 7}$. We study their chiral transformation properties. Particularly, we use the tetraquark currents belonging to the "nonexotic" $[(\overline{\mathbf{3}}, \mathbf{3}) \oplus(\mathbf{3}, \overline{\mathbf{3}})]$ chiral multiplets to calculate the masses of light scalar mesons through QCD sum rule. The two-point correlation functions are calculated including all terms and only the connected parts (Weinberg (2013), Coleman (1985), and Page (2003)). The results are consistent with the experimental values.

\section{Introduction}

The quark model is very successful in explaining the hadron spectrum with simply using quark-antiquark mesons and three-quark baryons [1-5]. However, there are always multiquark components in the Fock-space expansion of hadron states [6-8]. Hence, it is useful to properly include these multiquark components if we want to use Quantum Chromodynamics (QCD), the theory of strong interactions, to investigate hadrons in an exact way. Besides these "exotic" components, multiquark states themselves are also important in order to understand the low-energy behavior of QCD. These subjects have been studied for more than thirties years by lots of theoretical and experimental physicists [9-38]. Particularly, the light scalar mesons are good candidates due to their tetraquark (or molecular) components.

The light scalar mesons $f_{0}(500)$ (or $\sigma(500)$ ), $\kappa(800)$, $a_{0}(980)$, and $f_{0}(980)$ compose a flavor nonet whose masses are all below $1 \mathrm{GeV}$ [9]. Although such mesons have been intensively studied for many years, their nature is still not fully understood $[39,40]$. In the conventional quark model, light scalar mesons have a $\bar{q} q$ configuration of ${ }^{3} P_{0}$. However, because of their internal $p$-wave orbital excitation, their masses should exceed $1 \mathrm{GeV}$ and the ordering should be $m_{\sigma} \sim m_{a_{0}}<m_{\kappa}<m_{f_{0}}$ [41], which is inconsistent with the experiments. In chiral models, light scalar mesons are very important because they are chiral partners of the NambuGoldstone bosons, $\pi, K, \eta$, and $\eta^{\prime}$ [41]. Their masses are expected to be less than those of the quark model because of their collective nature. Light scalar mesons are also considered as tetraquark states or molecular states or containing large tetraquark components [31-35, 42, 43]. Considering that the diquark (antidiquark) inside has strong attraction, their masses are expected to be less than $1 \mathrm{GeV}$ and the ordering is expected to be $m_{\sigma}<m_{\kappa}<m_{f_{0}, a_{0}}$, which is consistent with the experiments.

To study the multiquark components of the light scalar mesons, we can use group theoretical methods, which have been applied to study quark-antiquark mesons and threequark baryons [44-58]. Cohen and Ji studied the chiral structure of meson currents constructed using one quark and one antiquark fields and baryon currents constructed using three quark fields [52]. In this paper we shall follow their approaches and study the chiral structure of local scalar and pseudoscalar tetraquark currents. These tetraquark currents can be used in the QCD sum rule analyses [43, 59-61] as well as the Lattice QCD calculations [6, 62-68].

In our previous references, we have applied the method of the QCD sum rule to calculate masses of light scalar mesons using local tetraquark currents [42,69-71]. We systematically classified the scalar tetraquark currents and found that there are altogether as many as five independent scalar local 
tetraquark currents for each flavor structure. Therefore, right currents should be used in order to study light scalar mesons. This is also closely related to the internal structure of light scalar mesons. A similar question for the baryon case has been studied in [48-51] where there are three independent local baryon fields of flavor octet. Previously we chose some mixed currents which provided good QCD sum rule results [42]. Although we did not know the relation of these currents with the internal structure of light scalar mesons at that time, we found that studying the chiral structure of scalar tetraquark currents can be useful to answer this question.

In this paper we shall try to answer this question (which currents should be used in order to study light scalar mesons). We shall systematically study the chiral structure of light scalar mesons through local tetraquark currents which belong to the "nonexotic" $[(\overline{\mathbf{3}}, \mathbf{3}) \oplus(\mathbf{3}, \overline{3})]$ chiral multiplets. This chiral multiplet only contains flavor singlet and octet mesons, and it does not contain any meson having exotic flavor structure. Since there are no experimental signals observing scalar mesons having exotic flavors, we assume that all the nine light scalar mesons (or their dominant components) belong to this multiplet. Moreover, these nine light scalar mesons can together compose one $[(\overline{\mathbf{3}}, \mathbf{3}) \oplus(\mathbf{3}, \overline{\mathbf{3}})]$ chiral multiplet. To do a systematical study, we shall investigate both scalar and pseudoscalar tetraquark currents, since they are chiral partners. We shall also investigate tetraquark currents of flavor singlet, octet, $\mathbf{1 0}, \overline{\mathbf{1 0}}$, and $\mathbf{2 7}$, which can be useful for further studies. We shall use the left handed quark field $L_{A}^{a} \equiv q_{L A}^{a}=\left(\left(1-\gamma_{5}\right) / 2\right) q_{A}^{a}$ and the right handed quark field $R_{A}^{a} \equiv q_{R A}^{a}=\left(\left(1+\gamma_{5}\right) / 2\right) q_{A}^{a}$ to rewrite these currents. After making proper combinations we can clearly see their chiral structures.

In this paper we shall use the method of QCD sum rule to calculate the masses of light scalar mesons through local scalar tetraquark currents belonging to the "nonexotic" $[(\overline{3}, 3) \oplus(3, \overline{3})]$ chiral multiplets. One tetraquark current can be always written as a combination of meson-meson currents through Fierz transformation $\left(\mathscr{Q}(x)=\sum_{i j} C_{i j} \mathscr{B}_{i}(x) \mathscr{B}_{j}(x)\right)$, and so the two-point correlation function contain two parts: the disconnected parts,

$$
\begin{aligned}
& \langle\mathscr{Q}(x) Q(y)\rangle_{\text {disconn }} \\
& \quad=\sum_{i j k l} C_{i j} C_{k l}\left\langle\mathscr{B}_{i}(x) \mathscr{B}_{k}(y)\right\rangle_{0}\left\langle\mathscr{B}_{j}(x) \mathscr{B}_{l}(y)\right\rangle_{0},
\end{aligned}
$$

and the connected parts,

$$
\begin{aligned}
& \langle Q(x) \mathscr{Q}(y)\rangle_{\text {conn }} \\
& =\sum_{i j k l} C_{i j} C_{k l}\left\langle\mathscr{B}_{i}(x) \mathscr{B}_{j}(x) \mathscr{B}_{k}(y) \mathscr{B}_{l}(y)\right\rangle_{0, \text { conn }} .
\end{aligned}
$$

In this paper we shall use both of them to perform QCD sum rule analysis. However, as suggested by Weinberg in his recent reference [72-74] using the large $N_{c}$ approximation: "A one tetraquark pole can only appear in the final, connected, term," we shall also use (only) the connected parts to perform QCD sum rule analysis.

This paper is organized as follows. In Section 2 we investigate local tetraquark currents of flavor singlet and $J^{P}=0^{+}$, and others are listed in Appendix A. In Section 3 we study their chiral transformation properties, and results are partly listed in Appendix B. In Section 4 we use the method of QCD sum rule to study the light scalar mesons through local scalar tetraquark currents belonging to the "nonexotic" $[(\overline{\mathbf{3}}, \mathbf{3}) \oplus$ $(3, \overline{3})]$ chiral multiplets. However, the results depend much on the threshold value $s_{0}$ suggesting a large contribution from the meson-meson continuum, and so in Section 5 we use only the connected parts of the two-point correlation function to perform the QCD sum rule analyses. Section 6 is a summary.

\section{Scalar Tetraquark Currents of Flavor Singlet}

We write the flavor structure of tetraquarks and study local tetraquark currents of flavor singlet and $J^{P}=0^{+}$:

$$
\begin{aligned}
3 \otimes 3 \otimes \overline{3} \otimes \overline{3}= & (\overline{3} \oplus \mathbf{6}) \otimes(3 \oplus \overline{\mathbf{6}}) \\
= & (\overline{\mathbf{3}} \otimes 3) \oplus(\overline{\mathbf{3}} \otimes \overline{\mathbf{6}}) \oplus(\mathbf{6} \otimes 3) \oplus(\mathbf{6} \otimes \overline{\mathbf{6}}) \\
= & (\mathbf{1} \oplus \mathbf{8}) \oplus(\mathbf{8} \oplus \overline{\mathbf{1 0}}) \oplus(\mathbf{8} \oplus 10) \\
& \oplus(\mathbf{1} \oplus \mathbf{8} \oplus 27) .
\end{aligned}
$$

There are two possibilities to construct a flavor single tetraquark current: both of the diquark and antidiquark have the antisymmetric flavor structure $\overline{\mathbf{3}}_{F}(q q) \otimes \mathbf{3}_{F}(\bar{q} \bar{q}) \rightarrow \mathbf{1}_{F}$ or have the symmetric flavor structure $\mathbf{6}_{F}(q q) \otimes \overline{\mathbf{6}}_{F}(\bar{q} \bar{q}) \rightarrow \mathbf{1}_{F}$. Together with five sets of Dirac matrices, $1, \gamma_{5}, \gamma_{\mu}, \gamma_{\mu} \gamma_{5}$, and $\sigma_{\mu \nu}$, we find the following ten independent local tetraquark currents of flavor singlet and $J^{P}=0^{+}$:

$$
\begin{gathered}
\eta_{1}^{\mathrm{S}, S}=q_{A}^{a T} \mathbb{C} \gamma_{5} q_{B}^{b}\left(\bar{q}_{A}^{a} \gamma_{5} \mathbb{C} \bar{q}_{B}^{b T}-\bar{q}_{A}^{b} \gamma_{5} \mathbb{C} \bar{q}_{B}^{a T}\right), \\
\eta_{2}^{\mathrm{S}, S}=q_{A}^{a T} \mathbb{C} q_{B}^{b}\left(\bar{q}_{A}^{a} \mathbb{C} \bar{q}_{B}^{b T}-\bar{q}_{A}^{b} \mathbb{C} \bar{q}_{B}^{a T}\right), \\
\eta_{3}^{\mathrm{S}, S}=q_{A}^{a T} \mathbb{C} \sigma_{\mu \nu} q_{B}^{b}\left(\bar{q}_{A}^{a} \sigma^{\mu \nu} \mathbb{C} \bar{q}_{B}^{b T}+\bar{q}_{A}^{b} \sigma^{\mu \nu} \mathbb{C} \bar{q}_{B}^{a T}\right), \\
\eta_{4}^{\mathrm{S}, S}=q_{A}^{a T} \mathbb{C} \gamma_{5} q_{B}^{b}\left(\bar{q}_{A}^{a} \gamma_{5} \mathbb{C} \bar{q}_{B}^{b T}+\bar{q}_{A}^{b} \gamma_{5} \mathbb{C} \bar{q}_{B}^{a T}\right), \\
\eta_{5}^{\mathrm{S}, \mathbb{S}}=q_{A}^{a T} \mathbb{C} q_{B}^{b}\left(\bar{q}_{A}^{a} \mathbb{C} \bar{q}_{B}^{b T}+\bar{q}_{A}^{b} \mathbb{C} \bar{q}_{B}^{a T}\right), \\
\eta_{6}^{\mathrm{S}, \mathbb{S}}=q_{A}^{a T} \mathbb{C} \sigma_{\mu \nu} q_{B}^{b}\left(\bar{q}_{A}^{a} \sigma^{\mu \nu} \mathbb{C} \bar{q}_{B}^{b T}-\bar{q}_{A}^{b} \sigma^{\mu \nu} \mathbb{C} \bar{q}_{B}^{a T}\right), \\
\eta_{7}^{\mathrm{S}, S}=q_{A}^{a T} \mathbb{C} \gamma_{\mu} \gamma_{5} q_{B}^{b}\left(\bar{q}_{A}^{a} \gamma^{\mu} \gamma_{5} \mathbb{C} \bar{q}_{B}^{b T}-\bar{q}_{A}^{b} \gamma^{\mu} \gamma_{5} \mathbb{C} \bar{q}_{B}^{a T}\right), \\
\eta_{8}^{\mathrm{S}, S}=q_{A}^{a T} \mathbb{C} \gamma_{\mu} q_{B}^{b}\left(\bar{q}_{A}^{a} \gamma^{\mu} \mathbb{C} \bar{q}_{B}^{b T}+\bar{q}_{A}^{b} \gamma^{\mu} \mathbb{C} \bar{q}_{B}^{a T}\right), \\
\eta_{9}^{\mathrm{S}, S}=q_{A}^{a T} \mathbb{C} \gamma_{\mu} \gamma_{5} q_{B}^{b}\left(\bar{q}_{A}^{a} \gamma^{\mu} \gamma_{5} \mathbb{C} \bar{q}_{B}^{b T}+\bar{q}_{A}^{b} \gamma^{\mu} \gamma_{5} \mathbb{C} \bar{q}_{B}^{a T}\right), \\
\eta_{10}^{\mathrm{S}, \mathbb{S}}=q_{A}^{a T} \mathbb{C} \gamma_{\mu} q_{B}^{b}\left(\bar{q}_{A}^{a} \gamma^{\mu} \mathbb{C} \bar{q}_{B}^{b T}-\bar{q}_{A}^{b} \gamma^{\mu} \mathbb{C} \bar{q}_{B}^{a T}\right) .
\end{gathered}
$$

In these expressions the summation is taken over repeated indices $(a, b, \ldots$ for color indices, $A, B, \ldots$ for flavor indices, and $\mu, v, \ldots$ for Lorentz indices). The two superscripts $S$ and $\mathbb{S}$ denote scalar $\left(J^{P}=0^{+}\right)$and flavor singlet, respectively. In this paper we also need to use the following notations: $\mathbb{C}$ is the charge-conjugation operator; $\epsilon^{A B C}$ is the totally 
TABLE 1: Flavor singlet tetraquark currents of $J^{P}=0^{+}$, showing their chiral representations and chirality. The second and third columns show the flavor and color structures of the diquark and antidiquark inside, respectively.

\begin{tabular}{|c|c|c|c|c|}
\hline Currents & lavor & olor & $s$ & $\mathrm{Ch}$ \\
\hline$\eta_{1}^{\mathrm{S}, S}-\eta_{2}^{\mathrm{S}, S}$ & $\overline{3} \otimes 3$ & $\overline{3} \otimes 3$ & \multirow{2}{*}[(\mathbf{1},\mathbf{1})]{} & \multirow{2}{*}{$L L \bar{L} \bar{L}+R R \bar{R} \bar{R}$} \\
\hline, $\mathbb{S}-\eta_{5}^{S, S}$ & $6 \otimes \overline{6}$ & $6 \otimes \overline{6}$ & & \\
\hline$\eta_{1}^{\mathrm{S}, S}+\eta_{2}^{\mathrm{S}, \mathrm{S}}$ & $\overline{3} \otimes 3$ & $\overline{3} \otimes 3$ & {$[(\overline{3}, 3)$} & $L L \bar{R} \bar{R}+R R \bar{L} \bar{L}$ \\
\hline$\eta_{4}^{\mathrm{S}, \mathrm{S}}+\eta_{5}^{\mathrm{S}, \mathrm{S}}$ & $6 \otimes \overline{6}$ & $\mathbf{6} \otimes \overline{\mathbf{6}}$ & {$[(\mathbf{6}, \overline{\mathbf{6}})+(\overline{\mathbf{6}}, \mathbf{6})]$} & $L L \bar{R} \bar{R}+R R \bar{L} \bar{L}$ \\
\hline$\eta_{3}^{\mathrm{S}, \mathbb{S}}$ & $\overline{3} \otimes 3$ & $6 \otimes \overline{6}$ & {$[(\overline{3}, 3)+($} & $L L \bar{R} \bar{R}$ \\
\hline$\eta_{6}^{\text {s, S }}$ & $6 \otimes \overline{6}$ & $\overline{3} \otimes 3$ & {$[(\mathbf{6}, \overline{\mathbf{6}})$} & $L L \bar{R} \bar{R}+$ \\
\hline$\eta_{7}^{\mathrm{S}, \mathrm{S}}-\eta_{10}^{\mathrm{S}, \mathrm{S}}$ & mixed & $\overline{3} \otimes$ & \multirow{2}{*}[(1,1)]{} & \multirow{2}{*}{$L R \bar{L} \bar{R}+R L \bar{R} \bar{L}$} \\
\hline$\eta_{8}^{\mathrm{S}, \mathrm{S}}-\eta_{9}^{\mathrm{S}, \mathrm{S}}$ & mixed & $6 \otimes \overline{6}$ & & \\
\hline $2 \eta_{7}^{\mathrm{S}, \mathbb{S}}+\eta_{10}^{\mathrm{S}, \mathbb{S}}$ & mixed & $\overline{3} \otimes 3$ & \multirow{2}{*}[(8,8)+(8,8)]{} & \multirow{2}{*}{$L R \bar{L} \bar{R}+R L \bar{R} \bar{L}$} \\
\hline$S \mathbb{S} \quad S \mathbb{S}$ & mixed & $6 \otimes$ & & \\
\hline
\end{tabular}

antisymmetric tensor; $S_{P}^{A B C}(P=1 \cdots 10)$ are the normalized totally symmetric matrices; $\lambda_{\mathrm{N}}(N=1 \cdots 8)$ are the GellMann matrices; $S_{U}^{A B C D}(U=1 \cdots 27)$ are the matrices for the 27 flavor representation, as defined in [58].

Among the ten currents, five currents $\left(\eta_{1,2,3,7,8}^{\mathrm{S}, S}\right)$ contain diquarks and antidiquarks both having the antisymmetric flavor structure $\overline{\mathbf{3}} \otimes \mathbf{3}$, and the rest $\left(\eta_{4,5,6,9,10}^{\mathrm{S}, \mathbb{S}}\right)$ contain diquarks and antidiquarks both having the symmetric flavor structure $\mathbf{6} \otimes \overline{\mathbf{6}}$. We note that after fixing the flavor and Lorentz structure of the internal diquarks and antidiquarks, their color structure is also fixed through Pauli's exclusion principle, as shown in Table 1.

The chiral structure of tetraquarks is more complicated than their flavor structure:

$$
\begin{aligned}
& ((3,1) \oplus(1,3))^{2} \otimes((\overline{3}, 1) \oplus(1, \overline{3}))^{2} \\
& =((\overline{3}, 1) \oplus(1, \overline{3}) \oplus(6,1) \oplus(1,6) \oplus(3,3) \oplus(3,3)) \\
& \quad \otimes((3,1) \oplus(1,3) \oplus(\overline{6}, 1) \oplus(1, \overline{6}) \oplus(\overline{3}, \overline{3}) \oplus(\overline{3}, \overline{3})) .
\end{aligned}
$$

The full (expanded) expressions are shown in [58]. Among them, the following multiplets contain flavor singlet tetraquarks currents: $[(\mathbf{1}, \mathbf{1})],[(\overline{\mathbf{3}}, \mathbf{3}) \oplus(\mathbf{3}, \overline{\mathbf{3}})],[(\mathbf{6}, \overline{\mathbf{6}}) \oplus(\overline{\mathbf{6}}, \mathbf{6})]$, and $[(\mathbf{8}, \mathbf{8}) \oplus(\mathbf{8}, \mathbf{8})]$, as well as their mirror multiples. Tetraquarks of all flavors can be chiral partners of flavor singlet tetraquarks because of the exotic $[(\mathbf{8}, \mathbf{8}) \oplus(\mathbf{8}, \mathbf{8})]$ chiral multiplet.

To clearly see the chiral structure of (4), we use the lefthanded quark field $L_{A}^{a} \equiv q_{L A}^{a}=\left(\left(1-\gamma_{5}\right) / 2\right) q_{A}^{a}$ and the righthanded quark field $R_{A}^{a} \equiv q_{R A}^{a}=\left(\left(1+\gamma_{5}\right) / 2\right) q_{A}^{a}$ to rewrite these currents and then combine them properly:

$$
\begin{aligned}
\eta_{1}^{\mathrm{S}, \mathbb{S}}-\eta_{2}^{\mathrm{S}, \mathbb{S}}= & -2 L_{A}^{a T} \mathbb{C} L_{B}^{b}\left(\bar{L}_{A}^{a} \mathbb{C} \bar{L}_{B}^{b T}-\bar{L}_{A}^{b} \mathbb{C} \bar{L}_{B}^{a T}\right) \\
& -2 R_{A}^{a T} \mathbb{C} R_{B}^{b}\left(\bar{R}_{A}^{a} \mathbb{C} \bar{R}_{B}^{b T}-\bar{R}_{A}^{b} \mathbb{C} \bar{R}_{B}^{a T}\right),
\end{aligned}
$$

$$
\begin{aligned}
& \eta_{1}^{\mathrm{S}, S}+\eta_{2}^{\mathrm{S}, \mathbb{S}}=2 L_{A}^{a T} \mathbb{C} L_{B}^{b}\left(\bar{R}_{A}^{a} \mathbb{C} \bar{R}_{B}^{b T}-\bar{R}_{A}^{b} \mathbb{C} \bar{R}_{B}^{a T}\right) \\
& +2 R_{A}^{a T} \mathbb{C} R_{B}^{b}\left(\bar{L}_{A}^{a} \mathbb{C} \bar{L}_{B}^{b T}-\bar{L}_{A}^{b} \mathbb{C} \bar{L}_{B}^{a T}\right), \\
& \eta_{4}^{\mathrm{S}, \mathbb{S}}-\eta_{5}^{\mathrm{S}, \mathbb{S}}=-2 L_{A}^{a T} \mathbb{C} L_{B}^{b}\left(\bar{L}_{A}^{a} \mathbb{C} \bar{L}_{B}^{b T}+\bar{L}_{A}^{b} \mathbb{C} \bar{L}_{B}^{a T}\right) \\
& -2 R_{A}^{a T} \mathbb{C} R_{B}^{b}\left(\bar{R}_{A}^{a} \mathbb{C} \bar{R}_{B}^{b T}+\bar{R}_{A}^{b} \mathbb{C} \bar{R}_{B}^{a T}\right), \\
& \eta_{4}^{\mathrm{S}, \mathbb{S}}+\eta_{5}^{\mathrm{S}, \mathbb{S}}=2 L_{A}^{a T} \mathbb{C} L_{B}^{b}\left(\bar{R}_{A}^{a} \mathbb{C} \bar{R}_{B}^{b T}+\bar{R}_{A}^{b} \mathbb{C} \bar{R}_{B}^{a T}\right) \\
& +2 R_{A}^{a T} \mathbb{C} R_{B}^{b}\left(\bar{L}_{A}^{a} \mathbb{C} \bar{L}_{B}^{b T}+\bar{L}_{A}^{b} \mathbb{C} \bar{L}_{B}^{a T}\right), \\
& \eta_{3}^{\mathrm{S}, \mathbb{S}}=L_{A}^{a T} \mathbb{C} \sigma_{\mu \nu} L_{B}^{b}\left(\bar{R}_{A}^{a} \sigma_{\mu \nu} \mathbb{C} \bar{R}_{B}^{b T}+\bar{R}_{A}^{b} \sigma_{\mu \nu} \mathbb{C} \bar{R}_{B}^{a T}\right) \\
& +R_{A}^{a T} \mathbb{C} \sigma_{\mu \nu} R_{B}^{b}\left(\bar{L}_{A}^{a} \sigma_{\mu \nu} \mathbb{C} \bar{L}_{B}^{b T}+\bar{L}_{A}^{b} \sigma_{\mu \nu} \mathbb{C} \bar{L}_{B}^{a T}\right), \\
& \eta_{6}^{\mathrm{S}, \mathbb{S}}=L_{A}^{a T} \mathbb{C} \sigma_{\mu \nu} L_{B}^{b}\left(\bar{R}_{A}^{a} \sigma_{\mu \nu} \mathbb{C} \bar{R}_{B}^{b T}-\bar{R}_{A}^{b} \sigma_{\mu \nu} \mathbb{C} \bar{R}_{B}^{a T}\right) \\
& +R_{A}^{a T} \mathbb{C} \sigma_{\mu \nu} R_{B}^{b}\left(\bar{L}_{A}^{a} \sigma_{\mu \nu} \mathbb{C} \bar{L}_{B}^{b T}-\bar{L}_{A}^{b} \sigma_{\mu \nu} \mathbb{C} \bar{L}_{B}^{a T}\right), \\
& \eta_{7}^{\mathrm{S}, S}-\eta_{10}^{\mathrm{S}, \mathbb{S}}=-4 L_{A}^{a T} \mathbb{C} \gamma_{\mu} R_{B}^{b}\left(\bar{L}_{A}^{a} \gamma^{\mu} \mathbb{C} \bar{R}_{B}^{b T}-\bar{L}_{A}^{b} \gamma^{\mu} \mathbb{C} \bar{R}_{B}^{a T}\right), \\
& 2 \eta_{7}^{\mathrm{S}, S}+\eta_{10}^{\mathrm{S}, S}=3 \lambda_{N}^{C A} \lambda_{N}^{D B} L_{A}^{a T} \mathbb{C} \gamma_{\mu} R_{B}^{b} \\
& \times\left(\bar{L}_{C}^{a} \gamma^{\mu} \mathbb{C} \bar{R}_{D}^{b T}-\bar{L}_{C}^{b} \gamma^{\mu} \mathbb{C} \bar{R}_{D}^{a T}\right), \\
& \eta_{8}^{\mathrm{S}, \mathbb{S}}-\eta_{9}^{\mathrm{S}, \mathbb{S}}=4 L_{A}^{a T} \mathbb{C} \gamma_{\mu} R_{B}^{b}\left(\bar{L}_{A}^{a} \gamma^{\mu} \mathbb{C} \bar{R}_{B}^{b T}+\bar{L}_{A}^{b} \gamma^{\mu} \mathbb{C} \bar{R}_{B}^{a T}\right), \\
& 2 \eta_{8}^{\mathrm{S}, \mathbb{S}}+\eta_{9}^{\mathrm{S}, \mathbb{S}}=-3 \lambda_{N}^{C A} \lambda_{N}^{D B} L_{A}^{a T} \mathbb{C} \gamma_{\mu} R_{B}^{b} \\
& \times\left(\bar{L}_{C}^{a} \gamma^{\mu} \mathbb{C} \bar{R}_{D}^{b T}+\bar{L}_{C}^{b} \gamma^{\mu} \mathbb{C} \bar{R}_{D}^{a T}\right),
\end{aligned}
$$

from which we can quickly find out their chiral structure (representations). For example, $\eta_{1+2}^{\mathrm{S}, \mathbb{S}} \equiv \eta_{1}^{\mathrm{S}, \mathbb{S}}+\eta_{2}^{\mathrm{S}, \mathbb{S}}$ partly contains two left-handed quarks that have an antisymmetric flavor structure and two right-handed antiquarks that also have an antisymmetric flavor structure; therefore, this part has the chiral representation $(\overline{3}, \mathbf{3})$, and its full chiral representation is just $[(\overline{\mathbf{3}}, \mathbf{3})+(\mathbf{3}, \overline{\mathbf{3}})]$.

The results are listed in Table 1 . There are four chiral singlets $[(\mathbf{1}, \mathbf{1})]$, two $[(\overline{\mathbf{3}}, \mathbf{3})+(\mathbf{3}, \overline{\mathbf{3}})]$ chiral multiplets, two $[(\mathbf{6}, \overline{\mathbf{6}})+(\overline{\mathbf{6}}, \mathbf{6})]$ chiral multiplets, and two $[(\mathbf{8}, \mathbf{8})+(\mathbf{8}, \mathbf{8})]$ chiral multiplets. The $[(\overline{3}, 3)+(3, \overline{3})]$ chiral representation contains the Nambu-Goldstone bosons, $\pi, K$, $\eta$, and $\eta^{\prime}$ mesons, and it does not contain any meson having exotic flavor structure. Considering $\sigma$ and $\pi$ are believed to be chiral partners in chiral models, we assume that light scalar mesons belong to this "nonexotic" multiplet, and we shall concentrate on it in our subsequent analysis. Based on this assumption, we do not need to study other possible tetraquark states having exotic flavor structure. Moreover, the current $\eta_{3}^{S, \mathbb{S}}$ has the symmetric color structure $\mathbf{6} \otimes \overline{\mathbf{6}}$, where color interactions between quarks and antiquarks are repulsive. Therefore, it 
is questionable to use this current, but we shall still use it to perform the QCD sum rule analysis for comparison. We note that mixed currents used in [42] belong to the mixing of $[(\mathbf{1}, \mathbf{1})]$ and exotic $[(\mathbf{8}, \mathbf{8}) \oplus(\mathbf{8}, \mathbf{8})]$ multiplets.

To fully study this multiplet, the chiral partners of (4) are also studied, that is, the scalar and pseudoscalar tetraquark currents of flavor singlet, octet, 10, $\overline{\mathbf{1 0}}$, and 27 . The results are shown in Appendix A. The conventional pseudoscalar and scalar mesons made by one $\bar{q} q$ pair can also belong to the $[(3, \overline{3}) \oplus(\overline{3}, 3)]$ chiral multiplet. However, all the scalar tetraquark currents inside this multiplet have the $\mathrm{q}_{L} q_{L} \bar{q}_{R} \bar{q}_{R}+q_{R} q_{R} \bar{q}_{L} \bar{q}_{L}$ chirality, and so they are not direct chiral partners of these $\bar{q} q$ mesons addressed by chiral singlet quark-antiquark pairs, which have the $\left(\bar{q}_{L} q_{R}+\bar{q}_{R} q_{L}\right) \otimes\left(\bar{q}_{L} q_{L}+\right.$ $\bar{q}_{R} q_{R}$ ) chirality ("chiral" Fock-space expansion), unless these two types of chirality mix with each other. Similarly, all the pseudoscalar tetraquark currents inside this multiplet have the same $q_{L} q_{L} \bar{q}_{R} \bar{q}_{R}+q_{R} q_{R} \bar{q}_{L} \bar{q}_{L}$ chirality, and so they are not (direct) terms in the "chiral" Fock-space expansion of the $\bar{q} q$ pseudoscalar mesons ( $\pi$, etc.).

\section{Chiral Transformations}

We can study their chiral transformation properties to verify which currents are chiral partners. Under the $\mathbf{U}(\mathbf{1})_{\mathrm{V}}, \mathbf{U}(\mathbf{1})_{\mathrm{A}}$, $\mathrm{SU}(3)_{\mathrm{V}}$, and $\mathrm{SU}(3)_{\mathrm{A}}$ chiral transformations, the quark field, $q=q_{L}+q_{R}$, transforms as

$$
\begin{gathered}
\mathbf{U}(\mathbf{1})_{\mathrm{V}}: q \longrightarrow \exp \left(i a^{0}\right) q=q+\delta q, \\
\mathbf{S U}(\mathbf{3})_{\mathrm{V}}: q \longrightarrow \exp (i \vec{\lambda} \cdot \vec{a}) q=q+\delta^{\vec{a}} q, \\
\mathbf{U}(\mathbf{1})_{\mathbf{A}}: q \longrightarrow \exp \left(i \gamma_{5} b^{0}\right) q=q+\delta_{5} q, \\
\mathbf{S U}(\mathbf{3})_{\mathbf{A}}: q \longrightarrow \exp \left(i \gamma_{5} \vec{\lambda} \cdot \vec{b}\right) q=q+\delta_{5}^{\vec{b}} q
\end{gathered}
$$

where $\vec{\lambda}$ are the eight Gell-Mann matrices, $a^{0}$ an infinitesimal parameter for the $\mathbf{U}(\mathbf{1})_{\mathrm{V}}$ transformation, $\vec{a}$ the octet of $\mathrm{SU}(3)_{\mathrm{V}}$ group parameters, $b^{0}$ an infinitesimal parameter for the $\mathbf{U}(\mathbf{1})_{\mathbf{A}}$ transformation, and $\vec{b}$ the octet of the chiral transformations.

The chiral transformation equations for these tetraquark currents can be calculated straightforwardly, and we only show the final results. The local scalar and pseudoscalar tetraquark currents have been classified in Section 2 and Appendix A. We find that there are four $[(1,1)]$ chiral multiplets:

$$
\begin{gathered}
\left(\eta_{1}^{\mathrm{S}, S}-\eta_{2}^{\mathrm{S}, \mathbb{S}}, \eta_{1}^{\mathrm{PS}, \mathbb{S}}-\eta_{2}^{\mathrm{PS}, \mathbb{S}}\right), \\
\left(\eta_{4}^{\mathrm{S}, \mathbb{S}}-\eta_{5}^{\mathrm{S}, \mathbb{S}}, \eta_{4}^{\mathrm{PS}, \mathbb{S}}-\eta_{5}^{\mathrm{PS}, \mathbb{S}}\right), \\
\left(\eta_{7}^{\mathrm{S}, \mathbb{S}}-\eta_{10}^{\mathrm{S}, \mathbb{S}}, \eta_{7}^{\mathrm{PS}, \mathbb{S}}-\eta_{10}^{\mathrm{PS}, \mathbb{S}}=0\right), \\
\left(\eta_{8}^{\mathrm{S}, \mathbb{S}}-\eta_{9}^{\mathrm{S}, \mathbb{S}}, \eta_{8}^{\mathrm{PS}, \mathbb{S}}-\eta_{9}^{\mathrm{PS}, \mathbb{S}}=0\right) ;
\end{gathered}
$$

there are two $[(3, \overline{3}) \oplus(\overline{3}, 3)]$ chiral multiplets (or mirror multiplets):

$$
\begin{gathered}
\left(\eta_{1}^{\mathrm{S}, \mathbb{S}}+\eta_{2}^{\mathrm{S}, \mathbb{S}}, \eta_{1}^{\mathrm{PS}, \mathbb{S}}+\eta_{2}^{\mathrm{PS}, \mathbb{S}}, \eta_{1, N}^{\mathrm{S}, \mathbb{O}}+\eta_{2, N}^{\mathrm{S}, \mathbb{O}}, \eta_{1, N}^{\mathrm{PS}, \mathbb{O}}+\eta_{2, N}^{\mathrm{PS}, \mathbb{Q}}\right) \\
\left(\eta_{3}^{\mathrm{S}, \mathbb{S}}, \eta_{3}^{\mathrm{PS}, \mathbb{S}}, \eta_{3, N}^{\mathrm{S}, \mathbb{O}}, \eta_{3, N}^{\mathrm{PS}, \mathbb{O}}\right) ;
\end{gathered}
$$

there are two $[(\mathbf{6}, \overline{\mathbf{6}}) \oplus(\overline{\mathbf{6}}, \mathbf{6})]$ chiral multiplets (or mirror multiplets):

$$
\begin{gathered}
\left(\eta_{4}^{\mathrm{S}, \mathbb{S}}+\eta_{5}^{\mathrm{S}, \mathbb{S}}, \eta_{4}^{\mathrm{PS}, \mathbb{S}}+\eta_{5}^{\mathrm{PS}, \mathbb{S}}, \eta_{4, N}^{\mathrm{S}, \mathbb{Q}}+\eta_{5, N}^{\mathrm{S}, \mathbb{Q}}, \eta_{4, N}^{\mathrm{PS}, \mathbb{O}}\right. \\
\left.+\eta_{5, N}^{\mathrm{PS}, \mathbb{O}}, \eta_{1, U}^{\mathrm{S}, \mathbb{U S}}+\eta_{2, U}^{\mathrm{S}, \mathbb{T S}}, \eta_{1, U}^{\mathrm{PS}, \mathbb{U S}}+\eta_{2, U}^{\mathrm{PS}, \mathbb{T S}}\right) \\
\left(\eta_{6}^{\mathrm{S}, \mathbb{S}}, \eta_{6}^{\mathrm{PS}, \mathbb{S}}, \eta_{6, N}^{\mathrm{S}, \mathbb{O}}, \eta_{6, N}^{\mathrm{PS}, \mathbb{O}}, \eta_{3, U}^{\mathrm{S}, \mathbb{U S}}, \eta_{3, U}^{\mathrm{PS}, \mathbb{U S}}\right) ;
\end{gathered}
$$

there are two $[(\mathbf{8}, \mathbf{8}) \oplus(\mathbf{8}, \mathbf{8})]$ chiral multiplets:

$$
\begin{aligned}
& \left(2 \eta_{7}^{\mathrm{S}, \mathbb{S}}+\eta_{10}^{\mathrm{S}, \mathbb{S}}, 2 \eta_{7}^{\mathrm{PS}, \mathbb{S}}+\eta_{10}^{\mathrm{PS}, \mathbb{S}}\right. \\
& \left.\quad=0,5 \eta_{7, N}^{\mathrm{S}, \mathbb{Q}}+\eta_{10, N}^{\mathrm{S}, \mathbb{O}}, \eta_{7, N}^{\mathrm{PS}, \mathbb{Q}}+\eta_{10, N}^{\mathrm{PS}, \mathbb{O}}, \eta_{5, U}^{\mathrm{S}, \mathbb{U S}}, \eta_{1, P}^{\mathrm{PS}, \mathbb{D}}, \eta_{2, P}^{\mathrm{PS}, \overline{\mathbb{D}}}\right), \\
& \left(2 \eta_{8}^{\mathrm{S}, \mathbb{S}}+\eta_{9}^{\mathrm{S}, \mathbb{S}}, 2 \eta_{8}^{\mathrm{PS}, \mathbb{S}}+\eta_{9}^{\mathrm{PS}, \mathbb{S}}\right. \\
& \left.=0,5 \eta_{8, N}^{\mathrm{S}, \mathbb{Q}}+\eta_{9, N}^{\mathrm{S}, \mathbb{O}}, \eta_{8, N}^{\mathrm{PS}, \mathbb{O}}+\eta_{9, N}^{\mathrm{PS}, \mathbb{Q}}, \eta_{4, U}^{\mathrm{S}, \mathbb{U S}}, \eta_{2, P}^{\mathrm{PS}, \mathbb{D}}, \eta_{1, P}^{\mathrm{PS}, \overline{\mathbb{D}}}\right) ;
\end{aligned}
$$

there are four $[(\mathbf{8}, \mathbf{1}) \oplus(\mathbf{1}, \mathbf{8})]$ chiral multiplets (or mirror multiplets):

$$
\begin{aligned}
& \left(\eta_{1, N}^{\mathrm{S}, \mathbb{Q}}-\eta_{2, N}^{\mathrm{S}, \mathbb{O}}, \eta_{1, N}^{\mathrm{PS}, \mathbb{O}}-\eta_{2, N}^{\mathrm{PS}, \mathbb{O}}\right), \\
& \left(\eta_{4, N}^{\mathrm{S}, \mathbb{Q}}-\eta_{5, N}^{\mathrm{S}, \mathbb{O}}, \eta_{4, N}^{\mathrm{PS}, \mathbb{Q}}-\eta_{5, N}^{\mathrm{PS}, \mathbb{O}}\right), \\
& \left(\eta_{7, N}^{\mathrm{S}, \mathbb{O}}-\eta_{10, N}^{\mathrm{S}, \mathbb{O}}, \eta_{7, N}^{\mathrm{PS}, \mathbb{O}}-\eta_{10, N}^{\mathrm{PS}, \mathbb{O}}\right), \\
& \left(\eta_{8, N}^{\mathrm{S}, \mathbb{O}}-\eta_{9, N}^{\mathrm{S}, \mathbb{O}}, \eta_{8, N}^{\mathrm{PS}, \mathbb{O}}-\eta_{9, N}^{\mathrm{PS}, \mathbb{O}}\right) ;
\end{aligned}
$$

there is only one $[(27, \mathbf{1}) \oplus(\mathbf{1}, \mathbf{2 7})]$ chiral multiplet:

$$
\left(\eta_{1, U}^{\mathrm{S}, \mathbb{\mathbb { S }}}-\eta_{2, U}^{\mathrm{S}, \mathbb{\mathbb { S }}}, \eta_{1, U}^{\mathrm{PS}, \mathbb{U S}}-\eta_{2, U}^{\mathrm{PS}, \mathbb{U S}}\right) .
$$

Their chiral transformation properties are shown in Appendix B, except those for the two $[(\overline{\mathbf{3}}, \mathbf{3}) \oplus(\mathbf{3}, \overline{\mathbf{3}})]$ chiral multiplets, which we show here. We use $\left(\eta_{(\overline{3}, 3)}^{\mathrm{S}, \mathbb{S}}, \eta_{(\overline{\mathbf{3}, 3})}^{\mathrm{PS}, \mathbb{S}}, \eta_{(\overline{\mathbf{3}, 3}, \mathrm{~S}, \mathrm{O}}^{\mathrm{S}}, \eta_{(\overline{\mathbf{3}}, \mathbf{3}), N}^{\mathrm{PS}, \mathbb{O}}\right)$ to denote these two multiplets, $\left(\eta_{1}^{\mathrm{S}, \mathrm{S}}+\eta_{2}^{\mathrm{S}, \mathbb{S}}, \eta_{1}^{\mathrm{PS}, \mathbb{S}}+\eta_{2}^{\mathrm{PS}, \mathbb{S}}, \eta_{1, N}^{\mathrm{S}, \mathbb{Q}}+\eta_{2, N}^{\mathrm{S}, \mathbb{O}}, \eta_{1, N}^{\mathrm{PS}, \mathbb{Q}}+\eta_{2, N}^{\mathrm{PS}, \mathbb{O}}\right)$ and $\left(\eta_{3}^{\mathrm{S}, \mathbb{S}}, \eta_{3}^{\mathrm{PS}, \mathbb{S}}, \eta_{3, N}^{\mathrm{S}, \mathbb{O}}, \eta_{3, N}^{\mathrm{PS}, \mathbb{O}}\right)$, and they have the same chiral transformation properties:

$$
\begin{aligned}
& \delta_{5} \eta_{(\overline{\mathbf{3}}, \mathbf{3})}^{\mathrm{S}(\mathrm{PS}), \mathbb{S}}=4 i b \eta_{(\overline{\mathbf{3}}, \mathbf{3})}^{\mathrm{PS}(\mathrm{S}), \mathbb{S}}, \\
& \delta^{\vec{a}} \eta_{(\overline{3}, 3)}^{S(\mathrm{PS}), \mathbb{S}}=0, \\
& \delta_{5}^{\vec{b}} \eta_{(\overline{\mathbf{3}}, \mathbf{3})}^{\mathrm{S}(\mathrm{PS}), \mathbb{S}}=4 i b^{N} \eta_{(\overline{\mathbf{3}}, \mathbf{3}), N}^{\mathrm{PS}(\mathrm{S}),} \\
& \delta_{5} \eta_{(\overline{\mathbf{3}}, \mathbf{3}), N}^{\mathrm{S}(\mathrm{PS}) \mathbb{Q}}=4 i b \eta_{(\overline{\mathbf{3}}, \mathbf{3}), N}^{\mathrm{PS}(\mathrm{S}), \mathbb{O}}, \\
& \delta^{\vec{a}} \eta_{(\overline{\mathbf{3}}, \mathbf{3}), N}^{\mathrm{S}(\mathrm{PS}), \mathbb{O}}=2 a^{N} f_{N M O} \eta_{(\overline{\mathbf{3}}, \mathbf{3}), \mathrm{O}}^{\mathrm{S}(\mathrm{PS}),}, \\
& \delta_{5}^{\vec{b}} \eta_{(\overline{\mathbf{3}}, \mathbf{3}), N}^{\mathrm{S}(\mathrm{PS}), \mathbb{O}}=\frac{2}{3} i b^{M} \eta_{(\overline{\mathbf{3}}, \mathbf{3})}^{\mathrm{PS}(\mathrm{S}), \mathbb{S}}-2 i b^{N} d_{N M O} \eta_{(\overline{\mathbf{3}}, \mathbf{3}), \mathrm{O}}^{\mathrm{PS}(\mathrm{S}), \mathbb{O}} .
\end{aligned}
$$


These chiral transformation equations can be compared to those calculated in [58] which have the same chirality and chiral representation, but in [58] only the flavor structure is taken into account. The $\mathbf{S U}(3)_{\mathbf{V}}$ and $\mathbf{S U}(3)_{\mathbf{A}}$ equations are similar to those of $\bar{q} q$ mesons as well as baryons belonging to the same chiral multiplet $[53,58]$, suggesting that chiral transformation properties are closely related to chiral representations, while $\mathbf{U}(1)_{\text {A }}$ equations are different, which may be reasons for the $U(1)_{A}$ anomaly.

The following formula obtained from [58] is used in the calculations:

$$
\begin{aligned}
\lambda_{C A}^{N} & \lambda_{D B}^{M} \\
= & -\frac{1}{12} \delta^{N M} \delta_{C A} \delta_{D B}+\frac{1}{4} \delta^{N M} \delta_{D A} \delta_{C B} \\
& -\frac{2}{5} d_{N M O} \delta_{C A} \lambda_{D B}^{O}+\left(\frac{3}{5} d_{N M O}+\frac{i}{3} f_{N M O}\right) \delta_{D A} \lambda_{C B}^{O} \\
& +\left(\frac{3}{5} d_{N M O}-\frac{i}{3} f_{N M O}\right) \delta_{C B} \lambda_{D A}^{O}-\frac{2}{5} d_{N M O} \delta_{D B} \lambda_{C A}^{O} \\
& +\left(\mathbf{T}_{8 \times 10}^{N}\right)_{M P}^{*} \epsilon_{A B E} S_{C D E}^{\mathbf{1 0}, P}+\left(\mathbf{T}_{8 \times 10}^{N}\right)_{M P} \epsilon_{C D E} S_{A B E}^{\mathbf{1 0 , P}} \\
& +\left(\mathbf{T}_{8 \times 27}^{N}\right)_{M U} S_{C D ; A B}^{27, U},
\end{aligned}
$$

as well as several other formulae:

$$
\begin{aligned}
& \epsilon_{A B E} S_{C G E}^{\mathbf{1 0} P} \lambda_{D G}^{N} \\
& =\mathbf{0}^{N P} \times \delta_{C A} \delta_{D B}+\mathbf{0}^{N P} \times \delta_{D A} \delta_{C B} \\
& -\frac{1}{3}\left(\mathbf{T}_{8 \times 10}^{N}\right)_{P O}^{T} \delta_{C A} \lambda_{D B}^{O}+\frac{2}{3}\left(\mathbf{T}_{8 \times 10}^{N}\right)_{P O}^{T} \delta_{D A} \lambda_{C B}^{O} \\
& +\frac{1}{3}\left(\mathbf{T}_{8 \times 10}^{N}\right)_{P O}^{T} \delta_{C B} \lambda_{D A}^{O}-\frac{2}{3}\left(\mathbf{T}_{8 \times 10}^{N}\right)_{P O}^{T} \delta_{D B} \lambda_{C A}^{O} \\
& +\left(\mathbf{T}_{10 \times 10}^{N}\right)_{P Q}^{T} \epsilon_{A B E} S_{C D E}^{\mathbf{1 0}, P}+\mathbf{0}^{N P Q} \times \epsilon_{C D E} S_{A B E}^{\mathbf{1 0}, P} \\
& +\mathbf{0}^{N P U} \times S_{C D ; A B}^{27, U}, \\
& S_{A G E}^{\mathbf{1 0 , P}} \epsilon_{C D E} \lambda_{G B}^{N} \\
& =\mathbf{0}^{N P} \times \delta_{C A} \delta_{D B}+\mathbf{0}^{N P} \times \delta_{D A} \delta_{C B} \\
& -\frac{1}{3}\left(\mathbf{T}_{8 \times 10}^{N}\right)_{P O}^{\dagger} \delta_{C A} \lambda_{D B}^{O}+\frac{1}{3}\left(\mathbf{T}_{8 \times 10}^{N}\right)_{P O}^{\dagger} \delta_{D A} \lambda_{C B}^{O} \\
& +\frac{2}{3}\left(\mathbf{T}_{8 \times 10}^{N}\right)_{P O}^{\dagger} \delta_{C B} \lambda_{D A}^{O}-\frac{2}{3}\left(\mathbf{T}_{8 \times 10}^{N}\right)_{P O}^{\dagger} \delta_{D B} \lambda_{C A}^{O} \\
& +\mathbf{0}^{N P Q} \epsilon_{A B E} S_{C D E}^{\mathbf{1 0}, P}+\left(\mathbf{T}_{10 \times 10}^{N}\right)_{P Q} \times \epsilon_{C D E} S_{A B E}^{\mathbf{1 0}, P} \\
& +\mathbf{0}^{N P U} \times S_{C D ; A B}^{27, U}
\end{aligned}
$$

$$
\begin{aligned}
& \epsilon_{A G E} S_{C D E}^{10, P} \lambda_{G B}^{N} \\
& =\mathbf{0}^{N P} \times \delta_{C A} \delta_{D B}+\mathbf{0}^{N P} \times \delta_{D A} \delta_{C B} \\
& +\frac{1}{15}\left(\mathbf{T}_{8 \times 10}^{N}\right)_{P O}^{T} \delta_{C A} \lambda_{D B}^{O}+\frac{1}{15}\left(\mathbf{T}_{8 \times 10}^{N}\right)_{P O}^{T} \delta_{D A} \lambda_{C B}^{O} \\
& -\frac{4}{15}\left(\mathbf{T}_{8 \times 10}^{N}\right)_{P O}^{T} \delta_{C B} \lambda_{D A}^{O}-\frac{4}{15}\left(\mathbf{T}_{8 \times 10}^{N}\right)_{P O}^{T} \delta_{D B} \lambda_{C A}^{O} \\
& -\frac{1}{2}\left(\mathbf{T}_{10 \times 10}^{N}\right)_{P Q}^{T} \epsilon_{A B E} S_{C D E}^{\mathbf{1 0 , P}}+\mathbf{0}^{N P Q} \times \epsilon_{C D E} S_{A B E}^{\mathbf{1 0 , P}} \\
& +\left(\mathbf{T}^{\mathbf{B}}{ }_{10 \times 27}\right)_{P U}^{N} S_{C D ; A B}^{27, U}, \\
& S_{A B E}^{10, P} \epsilon_{C G E} \lambda_{D G}^{N} \\
& =\mathbf{0}^{N P} \times \delta_{C A} \delta_{D B}+\mathbf{0}^{N P} \times \delta_{D A} \delta_{C B} \\
& +\frac{1}{15}\left(\mathbf{T}_{8 \times 10}^{N}\right)_{P O}^{\dagger} \delta_{C A} \lambda_{D B}^{O}-\frac{4}{15}\left(\mathbf{T}_{8 \times 10}^{N}\right)_{P O}^{\dagger} \delta_{D A} \lambda_{C B}^{O} \\
& +\frac{1}{15}\left(\mathbf{T}_{8 \times 10}^{N}\right)_{P O}^{\dagger} \delta_{C B} \lambda_{D A}^{O}-\frac{4}{15}\left(\mathbf{T}_{8 \times 10}^{N}\right)_{P O}^{\dagger} \delta_{D B} \lambda_{C A}^{O} \\
& +\mathbf{0}^{N P Q} \epsilon_{A B E} S_{C D E}^{\mathbf{1 0}, P}-\frac{1}{2}\left(\mathbf{T}_{10 \times 10}^{N}\right)_{P Q} \times \epsilon_{C D E} S_{A B E}^{\mathbf{1 0 , P}} \\
& +\left(\mathbf{T}^{\mathbf{A}}{ }_{10 \times 27}\right)_{P U}^{N} S_{C D ; A B}^{27, U}, \\
& S_{C D ; A G}^{27, U} \lambda_{G B}^{N} \\
& =\mathbf{0}^{N U} \times \delta_{C A} \delta_{D B}+\mathbf{0}^{N U} \times \delta_{D A} \delta_{C B} \\
& -\frac{1}{10}\left(\mathbf{T}_{8 \times 27}^{N}\right)_{U O}^{\dagger} \delta_{C A} \lambda_{D B}^{O}-\frac{1}{10}\left(\mathbf{T}_{8 \times 27}^{N}\right)_{U O}^{\dagger} \delta_{D A} \lambda_{C B}^{O} \\
& +\frac{2}{5}\left(\mathbf{T}_{8 \times 27}^{N}\right)_{U O}^{\dagger} \delta_{C B} \lambda_{D A}^{O}+\frac{2}{5}\left(\mathbf{T}_{8 \times 27}^{N}\right)_{U O}^{\dagger} \delta_{D B} \lambda_{C A}^{O} \\
& +\frac{3}{2}\left(\mathbf{T}^{\mathbf{B}}{ }_{10 \times 27}\right)_{U P}^{N \dagger} \times \epsilon_{A B E} S_{C D E}^{\mathbf{1 0 , P}}+\mathbf{0}^{N U P} \times \epsilon_{C D E} S_{A B E}^{\mathbf{1 0 , P}} \\
& +\left(\mathbf{T}_{27 \times 27}^{\mathbf{A}}\right)_{U V}^{N} S_{C D ; A B}^{27, U}, \\
& S_{C G ; A B}^{27, U} \lambda_{D G}^{N} \\
& =\mathbf{0}^{N U} \times \delta_{C A} \delta_{D B}+\mathbf{0}^{N U} \times \delta_{D A} \delta_{C B} \\
& -\frac{1}{10}\left(\mathbf{T}_{8 \times 27}^{N}\right)_{U O}^{\dagger} \delta_{C A} \lambda_{D B}^{O}+\frac{2}{5}\left(\mathbf{T}_{8 \times 27}^{N}\right)_{U O}^{\dagger} \delta_{D A} \lambda_{C B}^{O} \\
& -\frac{1}{10}\left(\mathbf{T}_{8 \times 27}^{N}\right)_{U O}^{\dagger} \delta_{C B} \lambda_{D A}^{O}+\frac{2}{5}\left(\mathbf{T}_{8 \times 27}^{N}\right)_{U O}^{\dagger} \delta_{D B} \lambda_{C A}^{O} \\
& +\mathbf{0}^{N U P} \times \epsilon_{A B E} S_{C D E}^{10, P}+\frac{3}{2}\left(\mathbf{T}^{\mathbf{A}}{ }_{10 \times 27}\right)_{U P}^{N \dagger} \times \epsilon_{C D E} S_{A B E}^{\mathbf{1 0 , P}} \\
& +\left(\mathbf{T}^{\mathbf{B}}{ }_{27 \times 27}\right)_{U V}^{N} S_{C D ; A B}^{27, U} .
\end{aligned}
$$

The transition matrices $\mathbf{T}_{8 \times 10}^{N}$ and $\mathbf{T}_{8 \times 27}^{N}$ have been obtained and listed in [58]. We list the transition matrices $\mathbf{T}_{10 \times 10}$, $\mathbf{T}^{\mathbf{A}}{ }_{10 \times 27}$, and $\mathbf{T}^{\mathbf{B}}{ }_{10 \times 27}$ in Appendix C. However, the transition 
matrices $\mathbf{T}_{27 \times 27}^{\mathbf{A}}$ and $\mathbf{T}_{27 \times 27}^{\mathbf{B}}$ are omitted due to their long expressions.

\section{QCD Sum Rule Analysis}

For the past decades QCD sum rule has proven to be a powerful and successful nonperturbative method [75, 76]. In sum rule analyses, we consider two-point correlation functions:

$$
\Pi\left(q^{2}\right) \equiv i \int d^{4} x e^{i q x}\left\langle 0\left|T J(x) J^{\dagger}(0)\right| 0\right\rangle
$$

where $J(x)$ is an interpolating field (current) coupling to a tetraquark state. Here we shall choose the tetraquark currents studied in Section 2 and Appendix A. We compute $\Pi\left(q^{2}\right)$ in the operator product expansion (OPE) of QCD up to certain order in the expansion, which is then matched with a hadronic parametrization to extract information about hadron properties. At the hadron level, we express the correlation function in the form of the dispersion relation with a spectral function:

$$
\Pi\left(q^{2}\right)=\frac{1}{\pi} \int_{s_{<}}^{\infty} \frac{\operatorname{Im} \Pi(s)}{s-q^{2}-i \varepsilon} d s,
$$

where the integration starts from the mass square of all current quarks. The imaginal part of the two-point correlation function is

$$
\operatorname{Im} \Pi(s) \equiv \pi \sum_{n} \delta\left(s-M_{n}^{2}\right)\langle 0|\eta| n\rangle\left\langle n\left|\eta^{\dagger}\right| 0\right\rangle .
$$

For the second equation, as usual, we adopt a parametrization of one pole dominance for the ground state $Y(\langle 0|\eta(0)| Y\rangle \equiv$ $f_{Y}$, where $f_{Y}$ is the decay constant) and a continuum contribution. The sum rule analysis is then performed after the Borel transformation of the two expressions of the correlation functions (17) and (18):

$$
\Pi^{(\text {all })}\left(M_{B}^{2}\right) \equiv \mathscr{B}_{M_{B}^{2}} \Pi\left(p^{2}\right)=\frac{1}{\pi} \int_{s_{<}}^{\infty} e^{-s / M_{B}^{2}} \operatorname{Im} \Pi(s) d s .
$$

Assuming the contribution from the continuum states can be approximated well by the spectral density of OPE above a threshold value $s_{0}$ (duality), we arrive at the sum rule equation

$$
f_{Y}^{2} e^{-M_{Y}^{2} / M_{B}^{2}}=\Pi\left(s_{0}, M_{B}^{2}\right) \equiv \frac{1}{\pi} \int_{s_{<}}^{s_{0}} e^{-s / M_{B}^{2}} \operatorname{Im} \Pi(s) d s .
$$

Differentiating (21) with respect to $1 / M_{B}^{2}$ and dividing it by (21), finally we obtain

$$
M_{Y}^{2}=\frac{\left(\partial / \partial\left(-1 / M_{B}^{2}\right)\right) \Pi\left(s_{0}, M_{B}^{2}\right)}{\Pi\left(s_{0}, M_{B}^{2}\right)} .
$$

The tetraquark currents classified in Section 2 and Appendix A can couple to mesons that belong to (or partly belong to) the same representation. Here, we assume that the scalar ones belonging to the "nonexotic" $[(\overline{3}, 3) \oplus(3, \overline{3})]$ chiral multiplets can couple to the light scalar mesons $f_{0}(500)$, $\kappa(800), a_{0}(980)$, and $f_{0}(980)$. Using these currents, we can calculate the mass of the light scalar mesons through the method of QCD sum rule. In the calculations, we assume an ideal mixing. Hence, the mass of the $f_{0}(500)$ meson is calculated through tetraquark currents:

$$
\begin{gathered}
\sigma_{1+2}=\frac{1}{6}\left(\eta_{1}^{\mathrm{S}, \mathbb{S}}+\eta_{2}^{\mathrm{S}, \mathbb{S}}\right)+\frac{1}{\sqrt{3}}\left(\eta_{1, N=8}^{\mathrm{S}, \mathbb{Q}}+\eta_{2, N=8}^{\mathrm{S}, \mathbb{Q}}\right), \\
\sigma_{3}=\frac{1}{6} \eta_{3}^{\mathrm{S}, \mathbb{S}}+\frac{1}{\sqrt{3}} \eta_{3, N=8}^{\mathrm{S}, \mathbb{Q}},
\end{gathered}
$$

whose quark contents are $u d \bar{u} \bar{d} ; \kappa^{+}(800)$ through

$$
\begin{gathered}
\kappa_{1+2}=\frac{1}{2}\left(\eta_{1, N=4}^{\mathrm{S}, \mathbb{Q}}+\eta_{2, N=4}^{\mathrm{S}, \mathbb{Q}}-i \eta_{1, N=5}^{\mathrm{S}, \mathbb{Q}}-i \eta_{2, N=5}^{\mathrm{S}, \mathbb{Q}}\right) \\
\kappa_{3}=\frac{1}{2}\left(\eta_{3, N=4}^{\mathrm{S}, \mathbb{Q}}-i \eta_{3, N=5}^{\mathrm{S}, \mathbb{Q}}\right)
\end{gathered}
$$

whose quark contents are $u d \bar{d} \bar{s} ; a_{0}^{+}(980)$ through

$$
\begin{gathered}
a 0_{1+2}=\frac{1}{2}\left(\eta_{1, N=1}^{\mathrm{S}, \mathbb{Q}}+\eta_{2, N=1}^{\mathrm{S}, \mathbb{Q}}-i \eta_{1, N=2}^{\mathrm{S}, \mathbb{Q}}-i \eta_{2, N=2}^{\mathrm{S}, \mathbb{Q}}\right), \\
a 0_{3}=\frac{1}{2}\left(\eta_{3, N=1}^{\mathrm{S}, \mathbb{Q}}-i \eta_{3, N=2}^{\mathrm{S}, \mathbb{Q}}\right),
\end{gathered}
$$

whose quark contents are $u s \bar{d} \bar{s} ; f_{0}(980)$ through

$$
\begin{gathered}
f 0_{1+2}=\frac{1}{3 \sqrt{2}}\left(\eta_{1}^{\mathrm{S}, \mathrm{S}}+\eta_{2}^{\mathrm{S}, S}\right)-\frac{1}{\sqrt{6}}\left(\eta_{1, N=8}^{\mathrm{S}, \mathbb{Q}}+\eta_{2, N=8}^{\mathrm{S}, \mathbb{Q}}\right), \\
f 0_{3}=\frac{1}{3 \sqrt{2}} \eta_{3}^{\mathrm{S}, \mathbb{S}}-\frac{1}{\sqrt{6}} \eta_{3, N=8}^{\mathrm{S}, \mathbb{Q}}
\end{gathered}
$$

whose quark contents are $u s \bar{u} \bar{s}+d s \bar{d} \bar{s}$.

They lead to the following QCD sum rules where we have computed the operator product expansion up to the eighth dimension:

$$
\begin{aligned}
& f_{\sigma_{1+2}}^{2} e^{-M_{\sigma_{1+2}}^{2} / M_{B}^{2}} \\
& \quad=\Pi^{\sigma_{1+2}}\left(s_{0}, M_{B}^{2}\right) \\
& \quad=\int_{s_{<}}^{s_{0}} e^{-s / M_{B}^{2}} d s \times\left(\frac{1}{30720 \pi^{6}} s^{4}+\frac{\left\langle g^{2} G G\right\rangle}{3072 \pi^{6}} s^{2}\right), \\
& f_{\sigma_{3}}^{2} e^{-M_{\sigma_{3}}^{2} / M_{B}^{2}} \\
& \quad=\Pi^{\sigma_{3}}\left(s_{0}, M_{B}^{2}\right) \\
& \quad=\int_{s_{<}}^{s_{0}} e^{-s / M_{B}^{2}} d s \times\left(\frac{1}{1280 \pi^{6}} s^{4}+\frac{11\left\langle g^{2} G G\right\rangle}{768 \pi^{6}} s^{2}\right),
\end{aligned}
$$




$$
\begin{aligned}
& f_{\kappa_{1+2}}^{2} e^{-M_{\kappa_{1+2}}^{2} / M_{B}^{2}} \\
& =\Pi^{\kappa_{1+2}}\left(s_{0}, M_{B}^{2}\right) \\
& =\int_{s_{<}}^{s_{0}} e^{-s / M_{B}^{2}} d s \\
& \quad \times\left(\frac{1}{30720 \pi^{6}} s^{4}-\frac{m_{s}^{2}}{1536 \pi^{6}} s^{3}+\left(\frac{\left\langle g^{2} G G\right\rangle}{3072 \pi^{6}}+\frac{m_{s}\langle\bar{s} s\rangle}{192 \pi^{4}}\right) s^{2}\right. \\
& \left.\quad-\frac{m_{s}^{2}\left\langle g^{2} G G\right\rangle}{1024 \pi^{6}} s+\frac{m_{s}\left\langle g^{2} G G\right\rangle\langle\bar{s} s\rangle}{768 \pi^{4}}\right),
\end{aligned}
$$$$
f_{\kappa_{3}}^{2} e^{-M_{\kappa_{3}}^{2} / M_{B}^{2}}
$$$$
=\Pi^{\kappa_{3}}\left(s_{0}, M_{B}^{2}\right)
$$$$
=\int_{s_{<}}^{s_{0}} e^{-s / M_{B}^{2}} d s
$$$$
\times\left(\frac{1}{1280 \pi^{6}} s^{4}-\frac{m_{s}^{2}}{64 \pi^{6}} s^{3}+\left(\frac{11\left\langle g^{2} G G\right\rangle}{768 \pi^{6}}+\frac{m_{s}\langle\bar{s} s\rangle}{8 \pi^{4}}\right) s^{2}\right.
$$$$
\left.-\frac{11 m_{s}^{2}\left\langle g^{2} G G\right\rangle}{256 \pi^{6}} s+\frac{11 m_{s}\left\langle g^{2} G G\right\rangle\langle\bar{s} s\rangle}{192 \pi^{4}}\right),
$$$$
f_{a 0_{1+2}}^{2} e^{-M_{a 0_{1+2}}^{2} / M_{B}^{2}}
$$$$
=\Pi^{a 0_{1+2}}\left(s_{0}, M_{B}^{2}\right)
$$$$
=\int_{s_{<}}^{s_{0}} e^{-s / M_{B}^{2}} d s
$$$$
\times\left(\frac{1}{30720 \pi^{6}} s^{4}-\frac{m_{s}^{2}}{768 \pi^{6}} s^{3}\right.
$$$$
+\left(\frac{\left\langle g^{2} G G\right\rangle}{3072 \pi^{6}}+\frac{m_{s}\langle\bar{s} s\rangle}{96 \pi^{4}}\right) s^{2}-\frac{m_{s}^{2}\left\langle g^{2} G G\right\rangle}{512 \pi^{6}} s
$$$$
\left.+\frac{m_{s}\left\langle g^{2} G G\right\rangle\langle\bar{s} s\rangle}{384 \pi^{4}}+\frac{m_{s}^{2}\left(4\langle\bar{q} q\rangle^{2}+\langle\bar{s} s\rangle^{2}\right)}{24 \pi^{2}}\right),
$$

$$
\begin{aligned}
f_{a 0_{3}}^{2} e^{-M_{a 0_{3}}^{2} / M_{B}^{2}} \\
=\Pi^{a 0_{3}}\left(s_{0}, M_{B}^{2}\right) \\
=\int_{s_{<}}^{s_{0}} e^{-s / M_{B}^{2}} d s \\
\quad \times\left(\frac{1}{1280 \pi^{6}} s^{4}-\frac{m_{s}^{2}}{32 \pi^{6}} s^{3}\right.
\end{aligned}
$$

$$
\begin{aligned}
& +\left(\frac{11\left\langle g^{2} G G\right\rangle}{768 \pi^{6}}+\frac{m_{s}\langle\bar{s} s\rangle}{4 \pi^{4}}\right) s^{2}-\frac{11 m_{s}^{2}\left\langle g^{2} G G\right\rangle}{128 \pi^{6}} s \\
& \left.+\frac{11 m_{s}\left\langle g^{2} G G\right\rangle\langle\bar{s} s\rangle}{96 \pi^{4}}+\frac{m_{s}^{2}\left(4\langle\bar{q} q\rangle^{2}+\langle\bar{s} s\rangle^{2}\right)}{\pi^{2}}\right) .
\end{aligned}
$$

In this expression we only show terms containing the strange current quark mass up to $m_{s}^{2}$, while we keep all terms in the calculations. We also keep the terms containing the up and down current quark masses in the calculations, although they are quite small and give little contribution [77]. We note that we do not include high dimension terms which can be important, particularly the tree-level term $\alpha_{s}\langle\bar{q} q\rangle^{4}[78,79]$.

In (27)-(32), many terms are cancelled, including condensates $\langle\bar{q} q\rangle^{2}$ and $\langle\bar{q} q\rangle\langle g \bar{q} G q\rangle$, which are usually much larger than others. Moreover, (27) shows that effects of gluons are significant in the OPE of the $\sigma$ meson since the up and down current quark masses are quite small. The sum rules for $f_{0}(980)$ are the same as those for $a_{0}(980)$, and so we obtain the same mass for $a_{0}(980)$ and $f_{0}(980)$.

To perform the numerical analysis, we use the following values for the condensates and other parameters, which correspond to the energy scale of $1 \mathrm{GeV}[9,59,80-86]$ :

$$
\begin{gathered}
\langle\bar{q} q\rangle=-(0.240 \pm 0.010)^{3} \mathrm{GeV}^{3}, \\
\langle\bar{s} s\rangle=-(0.8 \pm 0.1) \times(0.240 \pm 0.010)^{3} \mathrm{GeV}^{3} \\
\left\langle g_{s}^{2} G G\right\rangle=(0.48 \pm 0.14) \mathrm{GeV}^{4} \\
m_{u}=2.90 \pm 0.20 \mathrm{MeV}, \quad m_{d}=6.35 \pm 0.27 \mathrm{MeV}, \\
m_{s}=125 \pm 20 \mathrm{MeV} \\
\left\langle g_{s} \bar{q} \sigma G q\right\rangle=-M_{0}^{2} \times\langle\bar{q} q\rangle \\
M_{0}^{2}=(0.8 \pm 0.2) \mathrm{GeV}^{2}
\end{gathered}
$$

As usual we assume the vacuum saturation for higher dimensional operators such as $\langle 0|\bar{q} q \bar{q} q| 0\rangle \sim\langle 0|\bar{q} q| 0\rangle\langle 0|\bar{q} q| 0\rangle$. There is a minus sign in the definition of the mixed condensate $\left\langle g_{s} \bar{q} \sigma G q\right\rangle$, which is different from that used in some other QCD sum rule studies. This difference just comes from the definition of coupling constant $g_{s}[80,81]$.

We use the current $\sigma_{1+2}$ as an example. First we extract its spectral density from (27) and show it in Figure 1 as a function of the energy $s$. It is almost positive definite, and so we can use it to perform QCD sum rule analyses. Then we need to study its OPE convergence. The Borel transformed correlation function of the current $\sigma_{1+2}$ is shown in Figure 2, when we take $s_{0}=0.4 \mathrm{GeV}^{2}$. We can clearly see that the $D=4$ terms give large contributions, and the convergence is good in the region $M_{B}>0.5 \mathrm{GeV}$, where OPEs are reliable. To fix the upper bound of the Borel window, we need to use the pole contribution, defined as the pole part divided by the sum of 


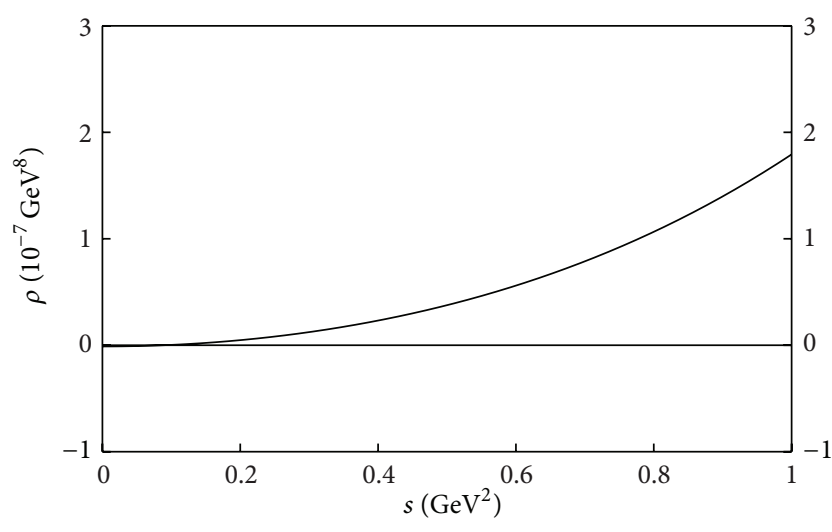

Figure 1: Spectral density for the current $\sigma_{1+2}$ as a function of the energy $s$.

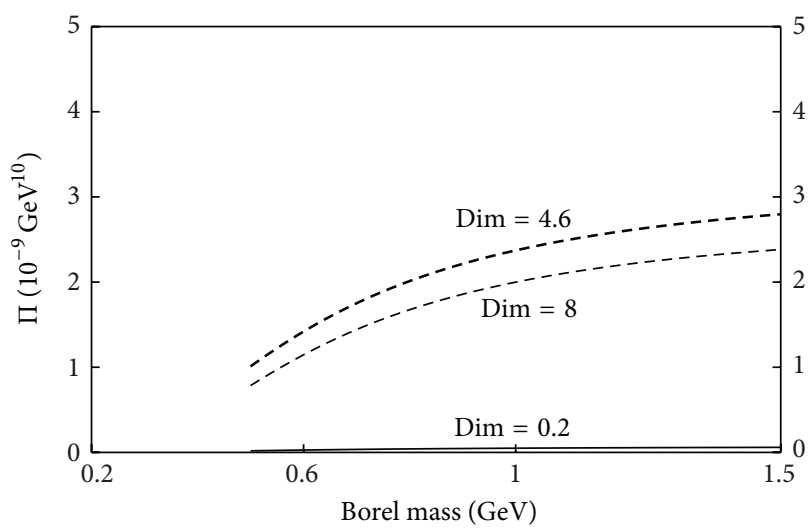

FIGURE 2: Various contributions to the correlation function of the current $\sigma_{1+2}$ as functions of the Borel mass $M_{B}$ in units of $\mathrm{GeV}^{10}$ at $s_{0}=0.4 \mathrm{GeV}^{2}$. The labels indicate the dimension up to which the OPE terms are included.

TABLE 2: Pole contributions of various currents.

\begin{tabular}{lccccccccc}
\hline & $\sigma_{1+2}$ & $\sigma_{1+2}^{\text {mod }}$ & $\sigma_{3}$ & $\kappa_{1+2}$ & $\kappa_{1+2}^{\text {mod }}$ & $\kappa_{3}$ & $a 0_{1+2}$ & $a 0_{1+2}^{\text {mod }}$ & $a 0_{3}$ \\
\hline$M_{B}(\mathrm{GeV})$ & 0.5 & 0.5 & 0.5 & 0.6 & 0.6 & 0.6 & 0.8 & 0.8 & 0.8 \\
$s_{0}\left(\mathrm{GeV}^{2}\right)$ & 0.4 & 0.4 & 0.4 & 0.9 & 0.9 & 0.9 & 1.8 & 1.8 & 1.8 \\
Pole $(\%)$ & 15 & 52 & 16 & 5 & 45 & 18 & 35 & 39 & 39 \\
\hline
\end{tabular}

the pole and the continuum parts in the two-point correlation function equation (17):

$$
\text { Pole contribution } \equiv \frac{\int_{0}^{s_{0}} e^{-s / M_{B}^{2}} \rho(s) d s}{\int_{0}^{\infty} e^{-s / M_{B}^{2}} \rho(s) d s} .
$$

It nearly vanishes for $\kappa(800)$ meson when using $\kappa_{1+2}$, as shown in Table 2 . For the $f_{0}(500)$ meson it is also not large. This suggests that the two-meson continuum contributes significantly. Only for the $a_{0}(980)$ and $f_{0}(980)$ mesons it is acceptable. Mathematically, this is because the continuum term is growing as $s^{4}$ and the condensates $\langle\bar{q} q\rangle^{2}$ and $\langle\bar{q} q\rangle\langle g \bar{q} G q\rangle$ cancelled.

We show masses of light scalar mesons as functions of the Borel mass $M_{B}$ and the threshold value $s_{0}$ in Figures 4 and 5,

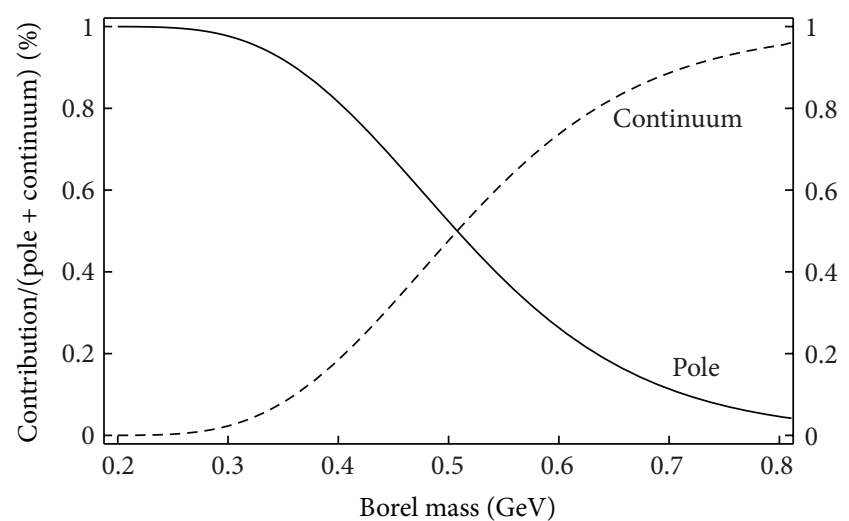

FIgURE 3: The solid curve shows the pole contribution and the dashed curve shows the continuum contribution (=1 - pole contribution).

using solid curves. The masses of $\sigma, \kappa$, and $a_{0}\left(f_{0}\right)$ are around $600 \mathrm{MeV}, 900 \mathrm{MeV}$, and $1100 \mathrm{MeV}$, respectively. However, these results much depend on threshold value $s_{0}$, especially for $\sigma$ and $a_{0}$, once more suggesting that the contribution of meson-meson continuum cannot be neglected. In such cases, the use of local quark-hadron duality with one resonance approximation is not valid.

In order to use the quark-hadron duality and obtain more reliable QCD sum rules, we should try to increase the pole contribution. This can be done by slightly changing the mixing parameters of currents $\eta_{1+2}$, which have the antisymmetric color structure $\overline{\mathbf{3}} \otimes \mathbf{3}$ and color interactions between quarks and antiquarks are repulsive (the details expressions are similar to (23), (24), (25), and (26)):

$$
\begin{aligned}
\sigma_{1+2} \sim & \eta_{1}^{\mathrm{S}, S(\mathbb{O})}+\eta_{2}^{\mathrm{S}, S(\mathbb{O})} \\
& \longrightarrow \sigma_{1+2}^{\bmod } \sim 0.99 \times \eta_{1}^{\mathrm{S}, \mathbb{S}(\mathbb{O})}+\eta_{2}^{\mathrm{S}, S(\mathbb{O})}, \\
\kappa_{1+2} \sim \eta_{1}^{\mathrm{S}, \mathbb{S}(\mathbb{O})}+\eta_{2}^{\mathrm{S}, S(\mathbb{O})} & \longrightarrow \kappa_{1+2}^{\bmod } \sim 0.99 \times \eta_{1}^{\mathrm{S}, \mathbb{S}(\mathbb{O})}+\eta_{2}^{\mathrm{S}, \mathbb{S}(\mathbb{O})}, \\
a 0_{1+2} & \sim \eta_{1}^{\mathrm{S}, \mathbb{S}(\mathbb{O})}+\eta_{2}^{\mathrm{S}, \mathbb{S}(\mathbb{O})} \\
& \longrightarrow a 0_{1+2}^{\bmod } \sim 0.99 \times \eta_{1}^{\mathrm{S}, \mathbb{S}(\mathbb{O})}+\eta_{2}^{\mathrm{S}, \mathbb{S}(\mathbb{O})}, \\
f 0_{1+2} & \sim \eta_{1}^{\mathrm{S}, \mathbb{S}(\mathbb{O})}+\eta_{2}^{\mathrm{S}, \mathbb{S}(\mathbb{O})} \\
& \longrightarrow f 0_{1+2}^{\bmod } \sim 0.99 \times \eta_{1}^{\mathrm{S}, S(\mathbb{O})}+\eta_{2}^{\mathrm{S}, \mathbb{S}(\mathbb{O})} .
\end{aligned}
$$

We note that doing this we introduce a few $[(\mathbf{8}, \mathbf{1}) \oplus(\mathbf{1}, \mathbf{8})]$ components, which are still "nonexotic." Mathematically, the condensates $\langle\bar{q} q\rangle^{2}$ and $\langle\bar{q} q\rangle\langle g \bar{q} G q\rangle$ appear and contribute, although the mixing parameters are only slightly modified.

Still we use the current $\sigma_{1+2}^{\bmod }$ as an example. The comparison between pole and continuum contributions for $s_{0}=0.4 \mathrm{GeV}^{2}$ is shown in Figure 3 [87-89]. We find that the pole contribution is significantly increased to around $50 \%$ when $M_{B}$ is around $0.5 \mathrm{MeV}$, but it decreases very quickly as 

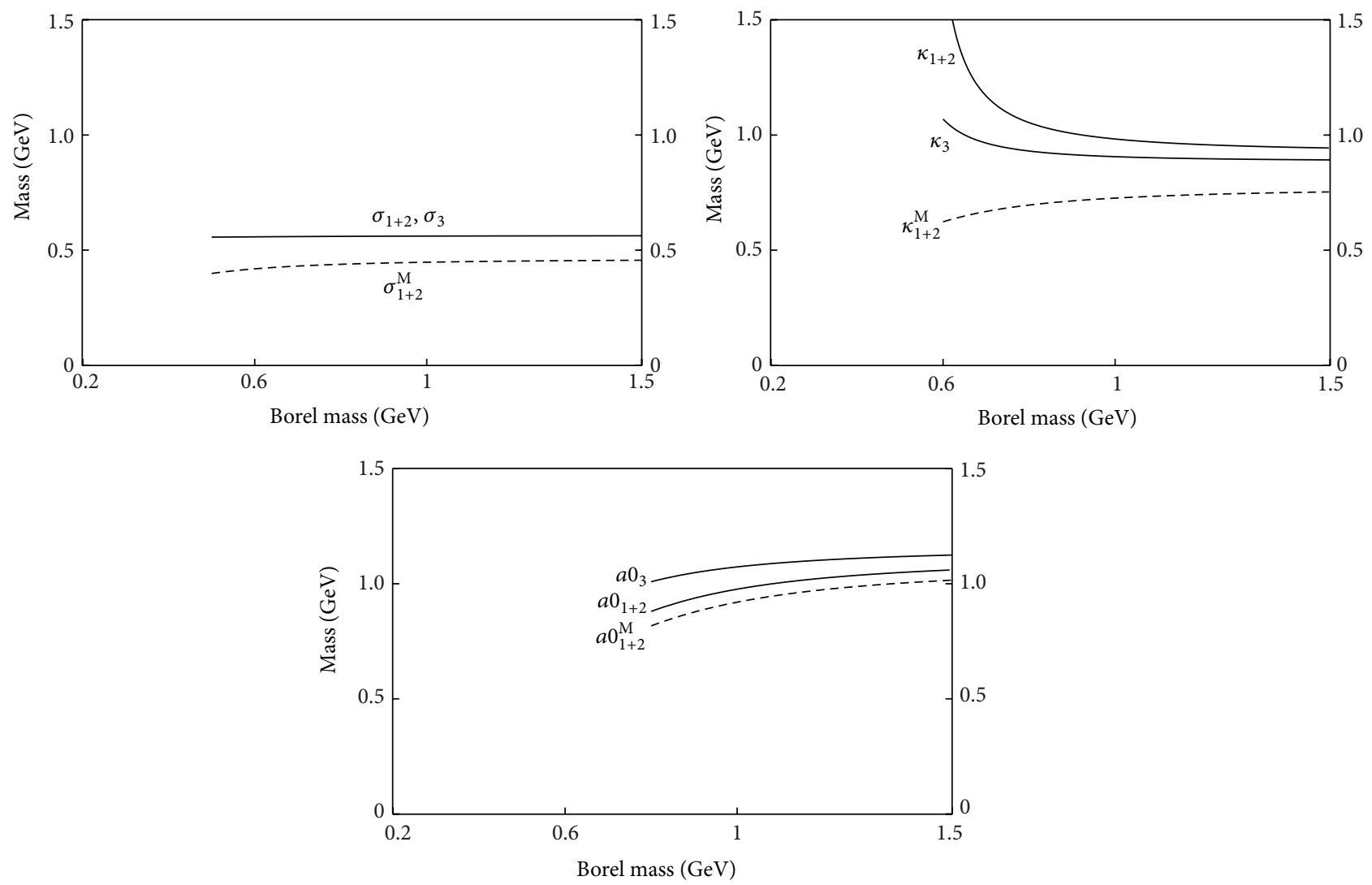

FIGURE 4: Mass of light scalar mesons $f_{0}(500), \kappa(800)$, and $a_{0}(980)\left(f_{0}(980)\right)$ as functions of Borel mass $M_{B}$. The threshold values $s_{0}$ for $f_{0}(500)$, $\kappa(800)$, and $a_{0}(980)\left(f_{0}(980)\right)$ are chosen to be $0.40,0.90$, and $1.80 \mathrm{GeV}^{2}$, respectively. The solid curves are obtained using the tetraquark currents $\sigma_{1+2}, \sigma_{3}, \kappa_{1+2}, \kappa_{3}, a 0_{1+2}$, and $a 0_{3}$ (see definitions in (23), (24), (25), and (26)), while the dashed curves are obtained using the modified currents $\sigma_{1+2}^{\text {mod }}, \kappa_{1+2}^{\text {mod }}$, and $a 0_{1+2}^{\text {mod }}$ (see definitions in (35)). The results for $f_{0}(980)$ are the same as those for $a_{0}(980)$.

the Borel mass increases. Therefore, we obtain a very narrow Borel window around $M_{B} \sim 0.5 \mathrm{GeV}$.

Using the modified currents listed in (35) we calculate masses of light scalar mesons. The results are shown in Figure 4 as functions of Borel mass $M_{B}$, but using dashed curves. The masses of $\sigma, \kappa$, and $a_{0}\left(f_{0}\right)$ are around $500 \mathrm{MeV}$, $700 \mathrm{MeV}$, and $900 \mathrm{MeV}$, respectively, better consistent with the experimental results. The pole contributions are significantly increased to be around $50 \%$ for $f_{0}(500)$ and $\kappa(800)$, as shown in Table 2. Since there is still $50 \%$ continuum and considering that nearly all the continuum comes from the $\pi-\pi$ contribution, the $\sigma$ meson is probably still contributed significantly by its underlying $\pi-\pi$ continuum.

We have also studied the threshold value $s_{0}$ dependence. The results are shown in Figure 5, using dashed lines. We can see that $s_{0}$ dependence is still significant suggesting the contribution of meson-meson continuum cannot be neglected. To solve this problem, we shall use only the connected parts of the two-point correlation function to perform the QCD sum rule analysis in the next section [72-74].

To investigate this meson-meson continuum we simply use the theory of relativity to estimate how far at most the two final pseudoscalar mesons can travel away from each other in the lifetimes of the initial light scalar mesons. From this distance we shall clearly see the difficulty to separate the meson-meson continuum. Our assumptions are very simple and straightforward: the initial state, an unstable particle $X$, is at rest in the beginning; it has mass $m_{X}$ and decay width $\Gamma_{X}$; it decays into two particles $A$ and $B$, having masses $m_{A}$ and $m_{B}$, respectively; when $X$ is decaying into $A$ and $B$, the mass difference between the initial and final states, $m_{X}-$ $m_{A}-m_{B}$, is totally and immediately transferred into kinetic energies of $A$ and $B$; this makes they have speeds $v_{A}$ and $v_{B}$, in opposite direction. This process can be easily described using the following equations:

$$
\begin{aligned}
& \frac{m_{A} c^{2}}{\sqrt{1-v_{A}^{2} / c^{2}}}+\frac{m_{B} c^{2}}{\sqrt{1-v_{B}^{2} / c^{2}}}=m_{X} c^{2}, \\
& \frac{m_{A}}{\sqrt{1-v_{A}^{2} / c^{2}}} v_{A}+\frac{m_{B}}{\sqrt{1-v_{B}^{2} / c^{2}}} v_{B}=0 .
\end{aligned}
$$

Here $c$ is the speed of light. The quantity $d_{X} \equiv\left(\hbar / \Gamma_{X}\right)\left(\left|v_{A}\right|+\right.$ $\left.\left|v_{B}\right|\right)$ is just the farthest distance that $A$ and $B$ can travel away from each other in the half-life of $X$. We can use the uncertainty principle $\Delta x \Delta p \geq \hbar / 2$ to estimate the theoretical uncertainty of $d_{X}$ : 

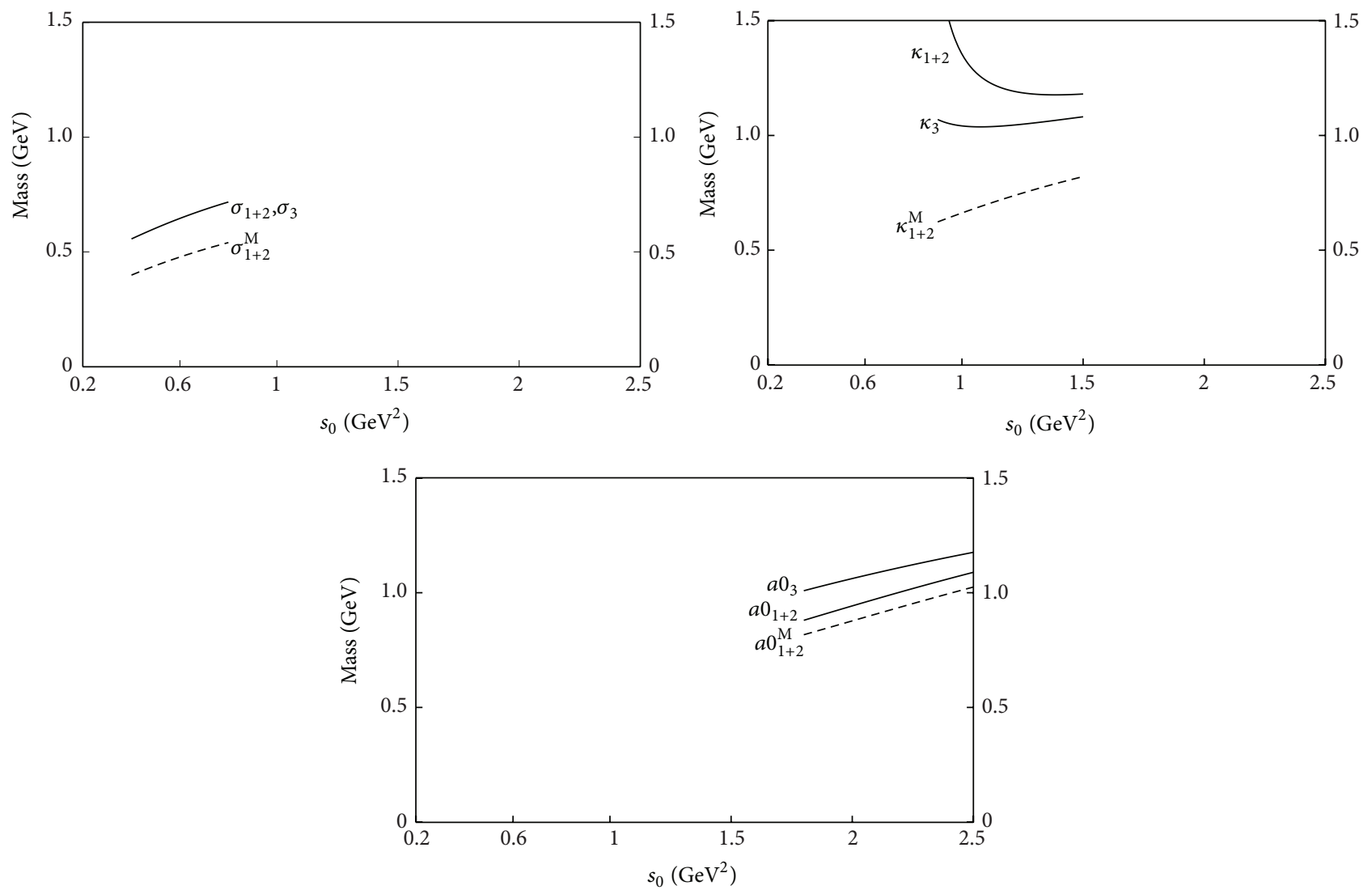

Figure 5: Mass of light scalar mesons $f_{0}(500), \kappa(800)$, and $a_{0}(980)\left(f_{0}(980)\right)$ as functions of the threshold value $s_{0}$. The Borel mass $M_{B}$ for $f_{0}(500), \kappa(800)$, and $a_{0}(980)\left(f_{0}(980)\right)$ are chosen to be $0.50,0.60$, and $0.80 \mathrm{GeV}^{2}$, respectively. The solid curves are obtained using the tetraquark currents $\sigma_{1+2}, \sigma_{3}, \kappa_{1+2}, \kappa_{3}, a 0_{1+2}$, and $a 0_{3}$ (see definitions in (23), (24), (25), and (26)), while the dashed curves are obtained using the modified currents $\sigma_{1+2}^{\text {mod }}, \kappa_{1+2}^{\text {mod }}$, and $a 0_{1+2}^{\text {mod }}$ (see definitions in (35)). The results for $f_{0}(980)$ are the same as those for $a_{0}(980)$.

$$
\Delta d_{X}=\frac{\hbar}{2} \frac{1}{\left(m_{A} / \sqrt{1-v_{A}^{2} / c^{2}}\right)\left|v_{A}\right|+\left(m_{B} / \sqrt{1-v_{B}^{2} / c^{2}}\right)\left|v_{B}\right|} .
$$

Using these equations we obtain: $d_{f_{0}(500) \rightarrow \pi \pi}=0.41-0.85 \mathrm{fm}$, $d_{\kappa(800) \rightarrow \pi K}=0.19-0.37 \mathrm{fm}, d_{a_{0}(980) \rightarrow \pi \eta}=2.76-5.69 \mathrm{fm}$, and $d_{f_{0}(980) \rightarrow \pi \pi}=3.79-9.50 \mathrm{fm}$. The relevant theoretical error bars from the uncertainty principle are $\Delta d_{f_{0}(500)}=$ $0.34 \mathrm{fm}, \Delta d_{\kappa(800)}=0.76 \mathrm{fm}, \Delta d_{a_{0}(980)}=0.16 \mathrm{fm}$, and $\Delta d_{f_{0}(980)}=0.11 \mathrm{fm}$. Moreover, in order to obtain these results we have assumed that the mass difference is totally and immediately transferred into kinetic energies, and so the actual distance that the two final states travel away from each other in the half-life of the initial unstable hadron can be even smaller. Therefore, in the cases of $f_{0}(500)$ and $\kappa(800)$, if the initial hadron is spherical in the beginning and the two final hadrons are both spherical in the end, the two final states may not separate geometrically even after the whole decay process. From this effect we clearly see that the meson-meson continuum contributes much in the cases of $f_{0}(500)$ and $\kappa(800)$, and it is quite difficulty to separate the meson-meson continuum. We note that this distance can be estimated for other hadrons, and this problem is not only for light scalar mesons.

\section{QCD Sum Rule Using Only Connected Parts}

In this section we use only the connected parts of the two-point correlation function to perform the QCD sum rule analysis. Using the large $N_{c}$ approximation, Weinberg suggested the following in his recent reference [72-74]: "A one tetraquark pole can only appear in the final, connected, term":

$$
\begin{aligned}
& \langle\mathscr{Q}(x) \mathscr{Q}(y)\rangle_{0} \\
& =\sum_{i j k l} C_{i j} C_{k l}\left[\left\langle\mathscr{B}_{i}(x) \mathscr{B}_{k}(y)\right\rangle_{0}\left\langle\mathscr{B}_{j}(x) \mathscr{B}_{l}(y)\right\rangle_{0}\right. \\
& \left.\quad+\left\langle\mathscr{B}_{i}(x) \mathscr{B}_{j}(x) \mathscr{B}_{k}(y) \mathscr{B}_{l}(y)\right\rangle_{\text {conn }}\right],
\end{aligned}
$$

where $Q(x)$ is a color-neutral operator, that is, a tetraquark current. Using Fierz transformation, it can be written in the form (see Appendix D for details)

$$
\mathcal{Q}(x)=\sum_{i j} C_{i j} \mathscr{B}_{i}(x) \mathscr{B}_{j}(x),
$$

and $\mathscr{B}_{i}(x)$ are color-neutral quark bilinears:

$$
\mathscr{B}_{i}(x)=\sum_{a} \overline{q_{a}^{A}(x)} \Gamma_{i} q_{a}^{B}(x) .
$$


In the previous section we have included both the connected parts (the second term in (39)) and the disconnected parts (the first term in (39)) to perform the QCD sum rule analysis. In this section we shall use only the connected parts. We shall use the same tetraquark currents. Although these currents are constructed using diquark and antidiquark fields, we do not need to change them to meson-meson form. We can simply select the connected parts in the contracted two-point correlation function. Take the current

$$
J(x)=\left[u_{a}^{T} C \gamma_{5} d_{b}\right]\left[\bar{u}_{a} \gamma_{5} C \bar{d}_{b}^{T}-\bar{u}_{b} \gamma_{5} C \bar{d}_{a}^{T}\right],
$$

as an example. We use $S_{a b}^{q}(x)$ to denote the quark propagator ( $q=u$ for up quark, and $q=d$ for down quark), and the contracted two-point correlation function is

$$
\begin{aligned}
\langle 0| J(x) & J^{\dagger}(0)|0\rangle \\
= & \operatorname{Tr}\left[\mathbb{C} S_{a 1 a 2}^{u T}(x) \mathbb{C} \gamma_{5} S_{b 1 b 2}^{d}(x) \gamma_{5}\right] \\
& \times \operatorname{Tr}\left[S_{a 2 a 1}^{u}(-x) \gamma_{5} \mathbb{C} S_{b 2 b 1}^{d T}(-x) \mathbb{C} \gamma_{5}\right] \\
& -\operatorname{Tr}\left[\mathbb{C} S_{a 1 a 2}^{u T}(x) \mathbb{C} \gamma_{5} S_{b 1 b 2}^{d}(x) \gamma_{5}\right] \\
& \times \operatorname{Tr}\left[S_{b 2 a 1}^{u}(-x) \gamma_{5} \mathbb{C} S_{a 2 b 1}^{d T}(-x) \mathbb{C} \gamma_{5}\right] \\
& -\operatorname{Tr}\left[\mathbb{C} S_{a 1 a 2}^{u T}(x) \mathbb{C} \gamma_{5} S_{b 1 b 2}^{d}(x) \gamma_{5}\right] \\
& \times \operatorname{Tr}\left[S_{a 2 b 1}^{u}(-x) \gamma_{5} \mathbb{C} S_{b 2 a 1}^{d T}(-x) \mathbb{C} \gamma_{5}\right] \\
& +\operatorname{Tr}\left[\mathbb{C} S_{a 1 a 2}^{u T}(x) \mathbb{C} \gamma_{5} S_{b 1 b 2}^{d}(x) \gamma_{5}\right] \\
& \times \operatorname{Tr}\left[S_{b 2 b 1}^{u}(-x) \gamma_{5} \mathbb{C} S_{a 2 a 1}^{d T}(-x) \mathbb{C} \gamma_{5}\right]
\end{aligned}
$$

where $a 1, a 2, b 1$, and $b 2$ are color indices. Its connected parts are just

$$
\begin{aligned}
\langle 0| J(x) & J^{\dagger}(0)|0\rangle_{\text {conn }} \\
= & -\operatorname{Tr}\left[\mathbb{C} S_{a 1 a 2}^{u T}(x) \mathbb{C} \gamma_{5} S_{b 1 b 2}^{d}(x) \gamma_{5}\right] \\
& \times \operatorname{Tr}\left[S_{b 2 a 1}^{u}(-x) \gamma_{5} \mathbb{C} S_{a 2 b 1}^{d T}(-x) \mathbb{C} \gamma_{5}\right] \\
& -\operatorname{Tr}\left[\mathbb{C} S_{a 1 a 2}^{u T}(x) \mathbb{C} \gamma_{5} S_{b 1 b 2}^{d}(x) \gamma_{5}\right] \\
& \times \operatorname{Tr}\left[S_{a 2 b 1}^{u}(-x) \gamma_{5} \mathbb{C} S_{b 2 a 1}^{d T}(-x) \mathbb{C} \gamma_{5}\right] .
\end{aligned}
$$

The tetraquark currents equations (23) to (26) lead to the following "connected" spectral densities:

$$
\begin{aligned}
& f_{\sigma_{1+2}}^{2} e^{-M_{\sigma_{1+2}}^{2} / M_{B}^{2}} \\
& \quad=\Pi^{\sigma_{1+2}}\left(s_{0}, M_{B}^{2}\right) \\
& \quad=\int_{s_{<}}^{s_{0}} e^{-s / M_{B}^{2}} d s \times\left(-\frac{1}{61440 \pi^{6}} s^{4}+\frac{\left\langle g^{2} G G\right\rangle}{3072 \pi^{6}}\right),
\end{aligned}
$$

$$
\begin{aligned}
& f_{\sigma_{3}}^{2} e^{-M_{\sigma_{3}}^{2} / M_{B}^{2}} \\
& =\Pi^{\sigma_{3}}\left(s_{0}, M_{B}^{2}\right) \\
& \quad=\int_{s_{<}}^{s_{0}} e^{-s / M_{B}^{2}} d s \times\left(\frac{1}{5120 \pi^{6}} s^{4}+\frac{5\left\langle g^{2} G G\right\rangle}{768 \pi^{6}}\right), \\
& f_{\mathcal{K}_{1+2}}^{2} e^{-M_{\kappa_{1+2}}^{2} / M_{B}^{2}} \\
& =\Pi^{\kappa_{1+2}}\left(s_{0}, M_{B}^{2}\right) \\
& =\int_{s_{<}}^{s_{0}} e^{-s / M_{B}^{2}} d s \\
& \quad \times\left(-\frac{1}{61440 \pi^{6}} s^{4}+\frac{m_{s}^{2}}{3072 \pi^{6}} s^{3}+\left(\frac{\left\langle g^{2} G G\right\rangle}{3072 \pi^{6}}-\frac{m_{s}\langle\bar{s} s\rangle}{384 \pi^{4}}\right) s^{2}\right. \\
& \left.\quad-\frac{m_{s}^{2}\left\langle g^{2} G G\right\rangle}{1024 \pi^{6}} s+\frac{m_{s}\left\langle g^{2} G G\right\rangle\langle\bar{s} s\rangle}{768 \pi^{4}}\right), \\
& f_{\mathcal{K}_{3}}^{2} e^{-M_{\kappa_{3}}^{2} / M_{B}^{2}} \\
& \quad=\Pi^{\kappa_{3}}\left(s_{0}, M_{B}^{2}\right) \\
& =\int_{s_{<}}^{s_{0}} e^{-s / M_{B}^{2}} d s \\
& \quad \times\left(\frac{1}{5120 \pi^{6}} s^{4}-\frac{m_{s}^{2}}{256 \pi^{6}} s^{3}+\left(\frac{5\left\langle g^{2} G G\right\rangle}{768 \pi^{6}}+\frac{m_{s}\langle\bar{s} s\rangle}{32 \pi^{4}}\right) s^{2}\right. \\
& \left.\quad-\frac{5 m_{s}^{2}\left\langle g^{2} G G\right\rangle}{256 \pi^{6}} s+\frac{5 m_{s}\left\langle g^{2} G G\right\rangle\langle\bar{s} s\rangle}{192 \pi^{4}}\right),
\end{aligned}
$$

$$
\begin{aligned}
& f_{a 0_{1+2}}^{2} e^{-M_{a 0_{1+2}}^{2} / M_{B}^{2}} \\
& =\Pi^{a 0_{1+2}}\left(s_{0}, M_{B}^{2}\right) \\
& =\int_{s_{<}}^{s_{0}} e^{-s / M_{B}^{2}} d s \\
& \quad \times\left(-\frac{1}{61440 \pi^{6}} s^{4}+\frac{m_{s}^{2}}{1536 \pi^{6}} s^{3}\right. \\
& \quad+\left(\frac{\left\langle g^{2} G G\right\rangle}{3072 \pi^{6}}-\frac{m_{s}\langle\bar{s} s\rangle}{192 \pi^{4}}\right) s^{2}-\frac{m_{s}^{2}\left\langle g^{2} G G\right\rangle}{512 \pi^{6}} s \\
& \left.\quad+\frac{m_{s}\left\langle g^{2} G G\right\rangle\langle\bar{s} s\rangle}{384 \pi^{4}}-\frac{m_{s}^{2}\left(4\langle\bar{q} q\rangle^{2}+\langle\bar{s} s\rangle^{2}\right)}{48 \pi^{2}}\right), \\
& f_{a 0_{3}}^{2} e^{-M_{a 0_{3}}^{2} / M_{B}^{2}} \\
& =\Pi^{a 0_{3}}\left(s_{0}, M_{B}^{2}\right)
\end{aligned}
$$




$$
\begin{aligned}
= & \int_{s_{<}}^{s_{0}} e^{-s / M_{B}^{2}} d s \\
& \times\left(\frac{1}{5120 \pi^{6}} s^{4}-\frac{m_{s}^{2}}{128 \pi^{6}} s^{3}\right. \\
& +\left(\frac{5\left\langle g^{2} G G\right\rangle}{768 \pi^{6}}+\frac{m_{s}\langle\bar{s} s\rangle}{16 \pi^{4}}\right) s^{2}-\frac{5 m_{s}^{2}\left\langle g^{2} G G\right\rangle}{128 \pi^{6}} s \\
& \left.+\frac{5 m_{s}\left\langle g^{2} G G\right\rangle\langle\bar{s} s\rangle}{96 \pi^{4}}+\frac{m_{s}^{2}\left(4\langle\bar{q} q\rangle^{2}+\langle\bar{s} s\rangle^{2}\right)}{4 \pi^{2}}\right) .
\end{aligned}
$$

The sum rules equations (45), (47), and (49) using tetraquark currents $\sigma_{3}, \kappa_{3}$, and $a 0_{3}\left(\mathrm{fO}_{3}\right)$ do not change significantly; that is, the connected and disconnected parts lead to similar results. This suggests that the meson-meson contribution is significant in both the connected and disconnected parts of these currents. We note that they have the symmetry color structure $\mathbf{6} \otimes \overline{\mathbf{6}}$, where color interactions between quarks and antiquarks are repulsive.

The sum rules equations (44), (46), and (48) do change significantly. Although the continuum term proportional to $s^{4}$ is negative, the spectral densities are positive in our working region $s \sim 1 \mathrm{GeV}^{2}$, as shown in Figure 6 for the spectral densities $\rho^{\sigma_{1+2}}, \rho^{\kappa_{1+2}}$, and $\rho^{a 0_{1+2}}$. We note that the pole contribution is not well defined because these spectral densities are negative when $s$ is large.

Masses of light scalar mesons are calculated using only the connected parts, and the results are shown in Figure 7, as functions of the Borel mass $M_{B}$ and the threshold value $s_{0}$. We clearly see that the Borel mass dependence is still not much; the mass of $\sigma$ still grows as the threshold value $s_{0}$ increases, suggesting that there is still much two-meson contribution (or related to its broad decay width); but the mass curves of $\kappa$ and $a_{0}$ have minimums around $s_{0}=0.8 \mathrm{GeV}^{2}$ for $\kappa$ and $s_{0}=1.3 \mathrm{GeV}^{2}$ for $a_{0}$, where the $s_{0}$ dependence is weak. We use these values as inputs, and calculate the masses of light scalar mesons.

Altogether there are two kinds of error bars: one is due to the two-meson continuum and the other is due to the $[(\mathbf{8}, \mathbf{1}) \oplus$ $(\mathbf{1}, \mathbf{8})$ ] components. This makes our results have large error bars: the mass of $f_{0}(500)$ is around $400 \sim 600 \mathrm{MeV}$, the mass of $\kappa(800)$ is around $700 \sim 900 \mathrm{MeV}$, and masses of $a_{0}(980)$ and $f_{0}(980)$ are around $900 \sim 1100 \mathrm{MeV}$.

\section{Summary}

We systematically studied the chiral structure of light scalar mesons using local scalar tetraquark currents that belong to the "nonexotic" $[(\overline{3}, 3) \oplus(\mathbf{3}, \overline{3})]$ chiral multiplets. This chiral representation only contains flavor singlet and octet mesons, and it does not contain any meson having exotic flavor structure. The nine light scalar mesons can just compose one $[(\overline{3}, 3) \oplus(3, \overline{3})]$ chiral multiplet. To do a systematical study, we investigated both scalar and pseudoscalar tetraquark currents, since they are chiral partners. We also investigated tetraquark currents of flavor singlet, octet, $\mathbf{1 0}, \overline{\mathbf{1 0}}$, and $\mathbf{2 7}$, which can be useful for further studies. Then we used the left handed quark field $L_{A}^{a} \equiv q_{L A}^{a}=\left(\left(1-\gamma_{5}\right) / 2\right) q_{A}^{a}$ and the right handed quark field $R_{A}^{a} \equiv q_{R A}^{a}=\left(\left(1+\gamma_{5}\right) / 2\right) q_{A}^{a}$ to rewrite these currents. After making proper combinations we verified their chiral representations.

We then used the QCD sum rule to calculate their masses. The masses of $\sigma, \kappa, a_{0}$, and $f_{0}$ are around $600 \mathrm{MeV}$, $900 \mathrm{MeV}, 1100 \mathrm{MeV}$, and $1100 \mathrm{MeV}$, respectively, generally consistent with the experimental values. However, the pole contributions are very small. Then we introduced a few $[(\mathbf{8}, \mathbf{1}) \oplus(\mathbf{1}, \mathbf{8})]$ components by slightly changing the mixing parameters from $\eta_{1}+\eta_{2} \rightarrow 0.99 \times \eta_{1}+\eta_{2}$. The masses of $\sigma, \kappa$, $a_{0}$, and $f_{0}$ are now around $500 \mathrm{MeV}, 700 \mathrm{MeV}, 900 \mathrm{MeV}$, and $900 \mathrm{MeV}$, respectively, better consistent with the experimental results. The pole contributions are significantly increased to be around $50 \%$ for $f_{0}(500)$ and $\kappa(800)$. However, these results still depend much on the threshold value $s_{0}$.

To solve this problem, we use only the connected parts of the two-point correlation function to perform the QCD sum rule analysis. We find that the results obtained using the tetraquark currents $\sigma_{3}, \kappa_{3}, a 0_{3}$, and $\mathrm{fO}_{3}$ (see (23)-(26)) do not change significantly. However, the results obtained using the tetraquark currents $\kappa_{1+2}, a 0_{1+2}$, and $f 0_{1+2}$ are improved: the mass curves of $\kappa$ and $a_{0}$ have minimums around $s_{0}=$ $0.8 \mathrm{GeV}^{2}$ for $\kappa$ and $s_{0}=1.3 \mathrm{GeV}^{2}$ for $a_{0}$, where the $s_{0}$ dependence is weak. We use these values as inputs and calculate the masses.

Altogether there are three kinds of error bars. The dominant one is due to the two-meson continuum. All light scalar mesons couple strongly to it, but we still do not know how to effectively separate them. The second one is due to the mixing of different chiral components. For example, we have included a few $[(\mathbf{8}, \mathbf{1}) \oplus(\mathbf{1}, \mathbf{8})]$ components to make our results reliable, but we do not know how much it is contained in light scalar mesons. The third one comes from our QCD sum rule calculations that we did not include the high dimensional terms, such as $\alpha_{s}\langle\bar{q} q\rangle^{4}$. Consequently, we obtained masses of light scalar mesons with large error bars: the mass of $f_{0}(500)$ is around $400 \sim 600 \mathrm{MeV}$, the mass of $\kappa(800)$ is around $700 \sim 900 \mathrm{MeV}$, and the mass of $a_{0}(980)$ and $f_{0}(980)$ is around $900 \sim 1100 \mathrm{MeV}$. We note that in [42] we used the same method to calculate masses of $\bar{q} q$ scalar mesons, which are all above $1 \mathrm{GeV}$.

We have also used these pseudoscalar tetraquark currents to perform the QCD sum rule analyses. For example, the one containing quark contents $q s \bar{q} \bar{s}$ has a mass around 1.3$1.6 \mathrm{GeV}$. This is significantly larger than the masses of the $\eta$ and $\eta^{\prime}$ mesons, suggesting that the Nambu-Goldstone bosons, $\pi, K, \eta$, and $\eta^{\prime}$, are predominantly $\bar{q} q$ states. We note that the finite decay width of light scalar mesons can be taken into account which does not change the final result significantly [42]. We also note that the contribution of instanton has not been considered in this paper whose effects can be significant since light scalar mesons have the same quantum numbers as vacuum. There are many papers discussing this [90-93]. 

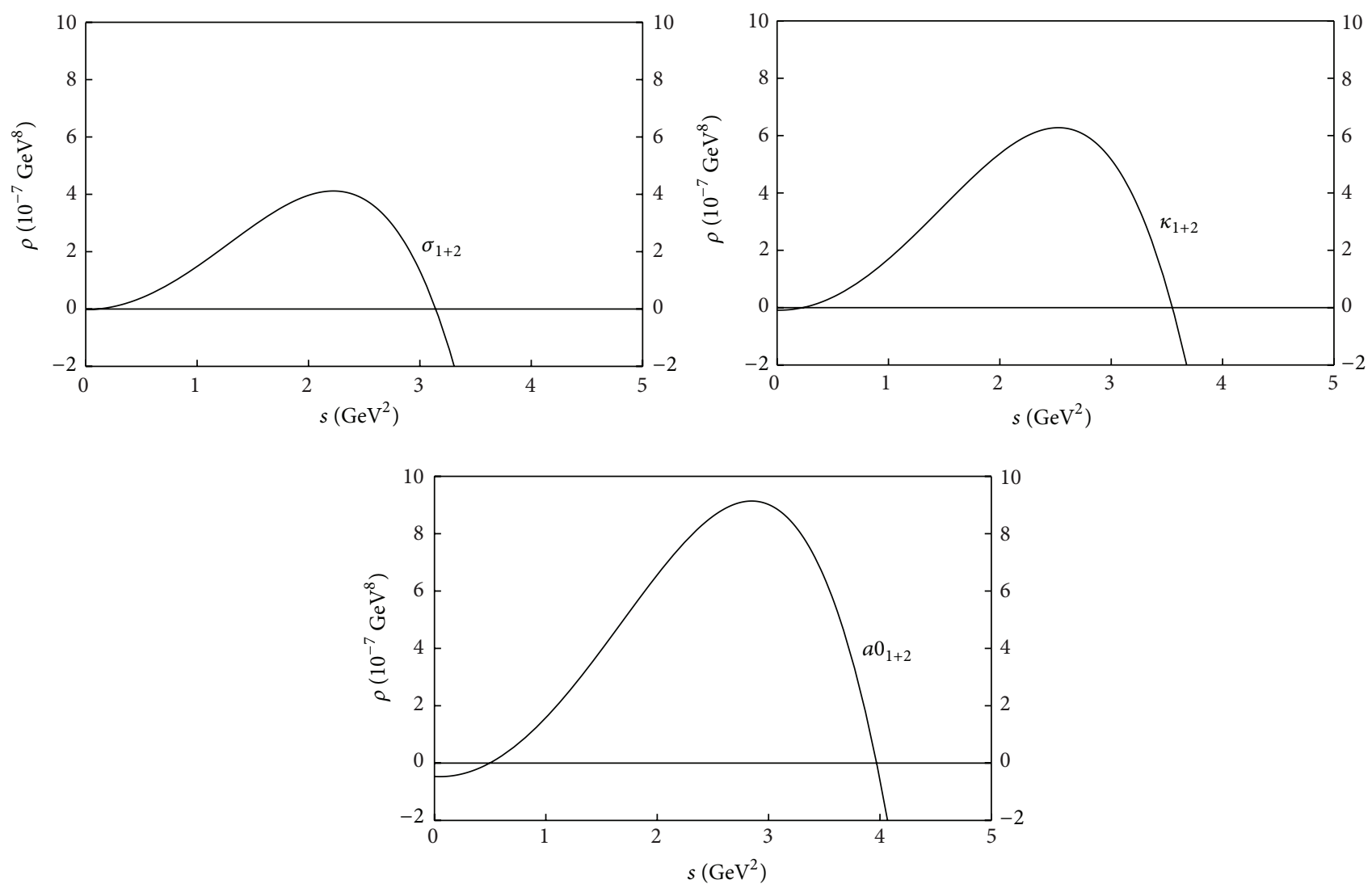

FIGURE 6: Spectral densities of light scalar mesons $f_{0}(500), \kappa(800)$, and $a_{0}(980)\left(f_{0}(980)\right)$ as functions of the energy $s$, where only the connected parts are taken into account.
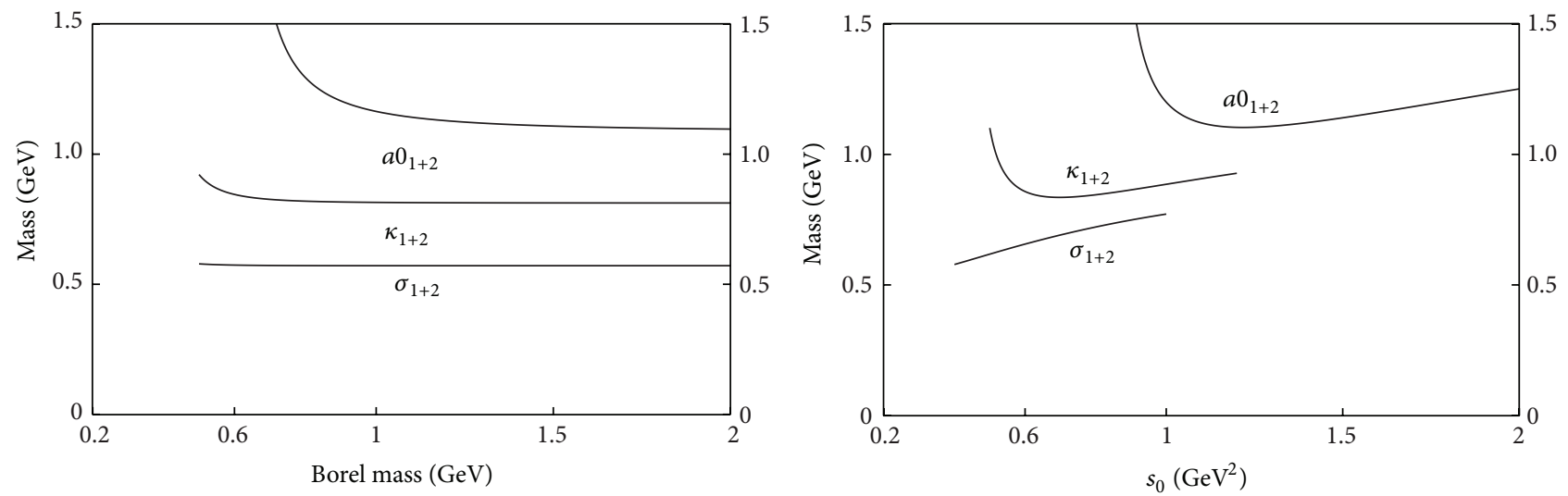

Figure 7: Masses of light scalar mesons $f_{0}(500), \kappa(800)$, and $a_{0}(800)\left(f_{0}(980)\right)$ as functions of the Borel mass $M_{B}$ and the threshold value $s_{0}$, where only the connected parts, (44), are taken into account. The threshold value $s_{0}$ for the left figure is chosen to be $0.4 \mathrm{GeV}^{2}, 0.8 \mathrm{GeV}^{2}$, and $1.3 \mathrm{GeV}^{2}$, while the Borel mass for the right figure is chosen to be $0.5 \mathrm{GeV}, 0.6 \mathrm{GeV}$, and $1.5 \mathrm{GeV}$, for $f_{0}(500), \kappa(800)$, and $a_{0}(800)\left(f_{0}(980)\right)$, respectively.

We note that we can also use the Fierz transformation to write tetraquark currents in a mesonic-mesonic form. Some relations are shown in Appendix D, and here we show one example:

$$
\begin{aligned}
\eta_{1}^{\mathrm{s}, \mathbb{S}}+\eta_{2}^{\mathrm{s}, \mathbb{S}}= & -\frac{1}{2}\left(\bar{q}_{A}^{a} \gamma_{5} q_{A}^{a}\right)\left(\bar{q}_{B}^{b} \gamma_{5} q_{B}^{b}\right) \\
& +\frac{1}{2}\left(\bar{q}_{A}^{a} \gamma_{5} q_{B}^{a}\right)\left(\bar{q}_{B}^{b} \gamma_{5} q_{A}^{b}\right)
\end{aligned}
$$

$$
\begin{aligned}
& -\frac{1}{2}\left(\bar{q}_{A}^{a} q_{A}^{a}\right)\left(\bar{q}_{B}^{b} q_{B}^{b}\right)+\frac{1}{2}\left(\bar{q}_{A}^{a} q_{B}^{a}\right)\left(\bar{q}_{B}^{b} q_{A}^{b}\right) \\
& +\frac{1}{4}\left(\bar{q}_{A}^{a} \sigma_{\mu \nu} q_{A}^{a}\right)\left(\bar{q}_{B}^{b} \sigma^{\mu \nu} q_{B}^{b}\right) \\
& -\frac{1}{4}\left(\bar{q}_{A}^{a} \sigma_{\mu \nu} q_{B}^{a}\right)\left(\bar{q}_{B}^{b} \sigma^{\mu v} q_{A}^{b}\right) \\
= & -\left[\left(\bar{q}_{L A}^{a} q_{R A}^{a}\right)\left(\bar{q}_{L B}^{b} q_{R B}^{b}\right)+\left(\bar{q}_{R A}^{a} q_{L A}^{a}\right)\left(\bar{q}_{R B}^{b} q_{L B}^{b}\right)\right]
\end{aligned}
$$




$$
\begin{aligned}
& +\left[\left(\bar{q}_{L A}^{a} q_{R B}^{a}\right)\left(\bar{q}_{L B}^{b} q_{R A}^{b}\right)+\left(\bar{q}_{R A}^{a} q_{L B}^{a}\right)\left(\bar{q}_{R B}^{b} q_{L A}^{b}\right)\right] \\
& +\frac{1}{4}\left[\left(\bar{q}_{L A}^{a} \sigma_{\mu \nu} q_{R A}^{a}\right)\left(\bar{q}_{L B}^{b} \sigma^{\mu \nu} q_{R B}^{b}\right)\right. \\
& \left.+\left(\bar{q}_{R A}^{a} \sigma_{\mu \nu} q_{L B}^{a}\right)\left(\bar{q}_{R B}^{b} \sigma^{\mu \nu} q_{L A}^{b}\right)\right] \\
& -\frac{1}{4}\left[\left(\bar{q}_{L A}^{a} \sigma_{\mu \nu} q_{R B}^{a}\right)\left(\bar{q}_{L B}^{b} \sigma^{\mu \nu} q_{R A}^{b}\right)\right. \\
& \left.+\left(\bar{q}_{R A}^{a} \sigma_{\mu \nu} q_{L A}^{a}\right)\left(\bar{q}_{R B}^{b} \sigma^{\mu \nu} q_{L B}^{b}\right)\right] .
\end{aligned}
$$

Considering $\bar{q}_{R} q_{L}$ and $\bar{q}_{L} q_{R}$ both belong to $[(\overline{\mathbf{3}}, 3) \oplus(3, \overline{\mathbf{3}})]$ representation (or its mirror), all local scalar tetraquark currents that belong to $[(\overline{\mathbf{3}}, \mathbf{3}) \oplus(\mathbf{3}, \overline{\mathbf{3}})]$ chiral multiplets are more similar to the combination of two $\bar{q} q$ mesons that both belong to this same representation. Consequently, the light scalar mesons are more similar to (like) tetraquarks or molecular states consisting two "non-chiral-singlet" $\bar{q} q$ mesons, unless different types of chirality mix with others.

The conventional pseudoscalar and scalar mesons made by one $\bar{q} q$ pair can also belong to the $[(\mathbf{3}, \overline{\mathbf{3}}) \oplus(\overline{\mathbf{3}}, \mathbf{3})]$ chiral multiplet. However, all the scalar tetraquark currents inside this multiplet have the $q_{L} q_{L} \bar{q}_{R} \bar{q}_{R}+q_{R} q_{R} \bar{q}_{L} \bar{q}_{L}$ chirality, and so they are not direct chiral partners of these $\bar{q} q$ mesons addressed by chiral singlet quark-antiquark pairs, which have the $\left(\bar{q}_{L} q_{R}+\bar{q}_{R} q_{L}\right) \otimes\left(\bar{q}_{L} q_{L}+\bar{q}_{R} q_{R}\right)$ chirality ("chiral" Fockspace expansion), unless these two types of chirality mix with each other; they are more similar to conventional $\bar{q} q$ mesons addressed by quark condensates; that is, mesons $(\bar{q} \Gamma q) \otimes$ condensates $\langle\bar{q} q\rangle=\left(\bar{q}_{L} q_{R}+\bar{q}_{R} q_{L}\right) \otimes\left(\bar{q}_{L} q_{R}+\bar{q}_{R} q_{L}\right)$.

Similarly, all the pseudoscalar tetraquark currents inside this multiplet also have the $q_{L} q_{L} \bar{q}_{R} \bar{q}_{R}+q_{R} q_{R} \bar{q}_{L} \bar{q}_{L}$ chirality, and so they are not (direct) terms in the "chiral" Fockspace expansion of the $\bar{q} q$ pseudoscalar mesons ( $\pi$, etc.). Therefore, in order to write the Fock-space expansion of the conventional pseudoscalar and scalar mesons, we probably need to study the mix of different types of chirality, which will be our next focus. In [72], Weinberg calculated the decay width of tetraquarks using the Large- $\mathrm{N}$ method. This can be done also using the method of QCD sum rule, which will be also our next focus.

\section{Appendices}

\section{A. Other Tetraquark Currents}

A.1. Pseudoscalar Tetraquark Currents of Flavor Singlet. In this subsection we study flavor singlet tetraquark currents of $J^{P}=0^{-}$. There are altogether six independent pseudoscalar currents as listed in the following:

$$
\begin{gathered}
\eta_{1}^{\mathrm{PS}, \mathbb{S}}=q_{A}^{a T} \mathbb{C} q_{B}^{b}\left(\bar{q}_{A}^{a} \gamma_{5} \mathbb{C} \bar{q}_{B}^{b T}-\bar{q}_{A}^{b} \gamma_{5} \mathbb{C} \bar{q}_{B}^{a T}\right), \\
\eta_{2}^{\mathrm{PS}, \mathbb{S}}=q_{A}^{a T} \mathbb{C} \gamma_{5} q_{B}^{b}\left(\bar{q}_{A}^{a} \mathbb{C} \bar{q}_{B}^{b T}-\bar{q}_{A}^{b} \mathbb{C} \bar{q}_{B}^{a T}\right), \\
\eta_{3}^{\mathrm{PS}, \mathbb{S}}=q_{A}^{a T} \mathbb{C} \sigma_{\mu \nu} q_{B}^{b}\left(\bar{q}_{A}^{a} \sigma^{\mu \nu} \gamma_{5} \mathbb{C} \bar{q}_{B}^{b T}+\bar{q}_{A}^{b} \sigma^{\mu \nu} \gamma_{5} \mathbb{C} \bar{q}_{B}^{a T}\right),
\end{gathered}
$$

$$
\begin{gathered}
\eta_{4}^{\mathrm{PS}, \mathbb{S}}=q_{A}^{a T} \mathbb{C} q_{B}^{b}\left(\bar{q}_{A}^{a} \gamma_{5} \mathbb{C} \bar{q}_{B}^{b T}+\bar{q}_{A}^{b} \gamma_{5} \mathbb{C} \bar{q}_{B}^{a T}\right), \\
\eta_{5}^{\mathrm{PS}, \mathbb{S}}=q_{A}^{a T} \mathbb{C} \gamma_{5} q_{B}^{b}\left(\bar{q}_{A}^{a} \mathbb{C} \bar{q}_{B}^{b T}+\bar{q}_{A}^{b} \mathbb{C} \bar{q}_{B}^{a T}\right), \\
\eta_{6}^{\mathrm{PS}, \mathbb{S}}=q_{A}^{a T} \mathbb{C} \sigma_{\mu \nu} q_{B}^{b}\left(\bar{q}_{A}^{a} \sigma^{\mu \nu} \gamma_{5} \mathbb{C} \bar{q}_{B}^{b T}-\bar{q}_{A}^{b} \sigma^{\mu \nu} \gamma_{5} C \bar{q}_{B}^{a T}\right) .
\end{gathered}
$$

We note that we can prove

$$
\begin{aligned}
& \eta_{7}^{\mathrm{PS}, \mathbb{S}}=\left(q_{A}^{a T} \mathbb{C} \gamma_{\mu} q_{B}^{b}\right)\left(\bar{q}_{A}^{a} \gamma^{\mu} \gamma_{5} \mathbb{C} \bar{q}_{B}^{b T}-\bar{q}_{A}^{b} \gamma^{\mu} \gamma_{5} \mathbb{C} \bar{q}_{B}^{a T}\right)=0, \\
& \eta_{8}^{\mathrm{PS}, \mathbb{S}}=\left(q_{A}^{a T} \mathbb{C} \gamma_{\mu} \gamma_{5} q_{B}^{b}\right)\left(\bar{q}_{A}^{a} \gamma^{\mu} \mathbb{C} \bar{q}_{B}^{b T}+\bar{q}_{A}^{b} \gamma^{\mu} \mathbb{C} \bar{q}_{B}^{a T}\right)=0, \\
& \eta_{9}^{\mathrm{PS}, \mathbb{S}}=\left(q_{A}^{a T} \mathbb{C} \gamma_{\mu} q_{B}^{b}\right)\left(\bar{q}_{A}^{a} \gamma^{\mu} \gamma_{5} \mathbb{C} \bar{q}_{B}^{b T}+\bar{q}_{A}^{b} \gamma^{\mu} \gamma_{5} \mathbb{C} \bar{q}_{B}^{a T}\right)=0, \\
& \eta_{10}^{\mathrm{PS}, \mathbb{S}}=\left(q_{A}^{a T} \mathbb{C} \gamma_{\mu} \gamma_{5} q_{B}^{b}\right)\left(\bar{q}_{A}^{a} \gamma^{\mu} \mathbb{C} \bar{q}_{B}^{b T}-\bar{q}_{A}^{b} \gamma^{\mu} \mathbb{C} \bar{q}_{B}^{a T}\right)=0 .
\end{aligned}
$$

The two superscripts PS and $\mathbb{S}$ denote pseudoscalar $\left(J^{P}=0^{-}\right)$ and flavor singlet, respectively. $\eta_{1,2,3}^{\mathrm{PS}, \mathbb{S}}$ contain diquarks and antidiquarks having the antisymmetric flavor structure $\overline{\mathbf{3}} \otimes \mathbf{3}$; $\eta_{4,5,6}^{\mathrm{PS}, \mathbb{S}}$ contain diquarks and antidiquarks having the symmetric flavor structure $\mathbf{6} \otimes \overline{\mathbf{6}}$. From the following combinations we can clearly see their chiral structure, where the left handed quark field $L_{A}^{a} \equiv q_{L A}^{a}=\left(\left(1-\gamma_{5}\right) / 2\right) q_{A}^{a}$ and the right handed quark field $R_{A}^{a} \equiv q_{R A}^{a}=\left(\left(1+\gamma_{5}\right) / 2\right) q_{A}^{a}$ are used:

$$
\begin{aligned}
& \eta_{1}^{\mathrm{PS}, \mathbb{S}}-\eta_{2}^{\mathrm{PS}, \mathbb{S}}=2 L_{A}^{a T} \mathbb{C} L_{B}^{b}\left(\bar{L}_{A}^{a} \mathbb{C} \bar{L}_{B}^{b T}-\bar{L}_{A}^{b} \mathbb{C} \bar{L}_{B}^{a T}\right) \\
& -2 R_{A}^{a T} \mathbb{C} R_{B}^{b}\left(\bar{R}_{A}^{a} \mathbb{C} \bar{R}_{B}^{b T}-\bar{R}_{A}^{b} \mathbb{C} \bar{R}_{B}^{a T}\right), \\
& \eta_{1}^{\mathrm{PS}, \mathbb{S}}+\eta_{2}^{\mathrm{PS}, \mathbb{S}}=-2 L_{A}^{a T} \mathbb{C} L_{B}^{b}\left(\bar{R}_{A}^{a} \mathbb{C} \bar{R}_{B}^{b T}-\bar{R}_{A}^{b} \mathbb{C} \bar{R}_{B}^{a T}\right) \\
& +2 R_{A}^{a T} \mathbb{C} R_{B}^{b}\left(\bar{L}_{A}^{a} \mathbb{C} \bar{L}_{B}^{b T}-\bar{L}_{A}^{b} \mathbb{C} \bar{L}_{B}^{a T}\right), \\
& \eta_{4}^{\mathrm{PS}, \mathbb{S}}-\eta_{5}^{\mathrm{PS}, \mathbb{S}}=2 L_{A}^{a T} \mathbb{C} L_{B}^{b}\left(\bar{L}_{A}^{a} \mathbb{C} \bar{L}_{B}^{b T}+\bar{L}_{A}^{b} \mathbb{C} \bar{L}_{B}^{a T}\right) \\
& -2 R_{A}^{a T} \mathbb{C} R_{B}^{b}\left(\bar{R}_{A}^{a} \mathbb{C} \bar{R}_{B}^{b T}+\bar{R}_{A}^{b} \mathbb{C} \bar{R}_{B}^{a T}\right), \\
& \eta_{4}^{\mathrm{PS}, \mathbb{S}}+\eta_{5}^{\mathrm{PS}, \mathbb{S}}=-2 L_{A}^{a T} \mathbb{C} L_{B}^{b}\left(\bar{R}_{A}^{a} \mathbb{C} \bar{R}_{B}^{b T}+\bar{R}_{A}^{b} \mathbb{C} \bar{R}_{B}^{a T}\right) \\
& +2 R_{A}^{a T} \mathbb{C} R_{B}^{b}\left(\bar{L}_{A}^{a} \mathbb{C} \bar{L}_{B}^{b T}+\bar{L}_{A}^{b} \mathbb{C} \bar{L}_{B}^{a T}\right), \\
& \eta_{3}^{\mathrm{PS}, \mathbb{S}}=-L_{A}^{a T} \mathbb{C} \sigma_{\mu \nu} L_{B}^{b}\left(\bar{R}_{A}^{a} \sigma_{\mu \nu} \mathbb{C} \bar{R}_{B}^{b T}+\bar{R}_{A}^{b} \sigma_{\mu \nu} \mathbb{C} \bar{R}_{B}^{a T}\right) \\
& +R_{A}^{a T} \mathbb{C} \sigma_{\mu \nu} R_{B}^{b}\left(\bar{L}_{A}^{a} \sigma_{\mu \nu} \mathbb{C} \bar{L}_{B}^{b T}+\bar{L}_{A}^{b} \sigma_{\mu \nu} \mathbb{C} \bar{L}_{B}^{a T}\right), \\
& \eta_{6}^{\mathrm{PS}, \mathbb{S}}=-L_{A}^{a T} \mathbb{C} \sigma_{\mu \nu} L_{B}^{b}\left(\bar{R}_{A}^{a} \sigma_{\mu \nu} \mathbb{C} \bar{R}_{B}^{b T}-\bar{R}_{A}^{b} \sigma_{\mu \nu} \mathbb{C} \bar{R}_{B}^{a T}\right) \\
& +R_{A}^{a T} \mathbb{C} \sigma_{\mu \nu} R_{B}^{b}\left(\bar{L}_{A}^{a} \sigma_{\mu \nu} \mathbb{C} \bar{L}_{B}^{b T}-\bar{L}_{A}^{b} \sigma_{\mu \nu} \mathbb{C} \bar{L}_{B}^{a T}\right) .
\end{aligned}
$$

We list their chirality and chiral representations in Table 3.

A.2. Scalar Tetraquark Currents of Flavor Octet. In this subsection we study flavor octet tetraquark currents of $J^{P}=0^{+}$. 
TABLE 3: Flavor singlet tetraquark currents of $J^{P}=0^{-}$classified in Appendix A.1.

\begin{tabular}{lcc}
\hline $\begin{array}{l}\text { Tetraquark currents of } \\
\left(\mathbf{1}_{F}, J^{P}=0^{-}\right)\end{array}$ & $\begin{array}{c}\text { Chiral } \\
\text { representations }\end{array}$ & Chirality \\
\hline$\eta_{1}^{\mathrm{PS}, S}-\eta_{2}^{\mathrm{PS}, \mathbb{S}}, \eta_{4}^{\mathrm{PS}, \mathbb{S}}-\eta_{5}^{\mathrm{PS}, S}$ & {$[(\mathbf{1}, \mathbf{1})+(\mathbf{1}, \mathbf{1})]$} & $L L \bar{L} \bar{L}+R R \bar{R} \bar{R}$ \\
$\eta_{1}^{\mathrm{PS}, S}+\eta_{2}^{\mathrm{PS}, \mathbb{S}}$ & {$[(\overline{\mathbf{3}}, \mathbf{3})+(\mathbf{3}, \overline{\mathbf{3}})]$} & $L L \bar{R} \bar{R}+R R \bar{L} \bar{L}$ \\
$\eta_{4}^{\mathrm{PS}, \mathbb{S}}+\eta_{5}^{\mathrm{PS}, \mathbb{S}}$ & {$[(\mathbf{6}, \overline{\mathbf{6}})+(\overline{\mathbf{6}}, \mathbf{6})]$} & $L L \bar{R} \bar{R}+R R \bar{L} \bar{L}$ \\
$\eta_{3}^{\mathrm{PS}, \mathbb{S}}$ & {$[(\overline{\mathbf{3}}, \mathbf{3})+(\mathbf{3}, \overline{\mathbf{3}})]$} & $L L \bar{R} \bar{R}+R R \bar{L} \bar{L}$ \\
$\eta_{6}^{\mathrm{PS}, \mathbb{S}}$ & {$[(\mathbf{6}, \overline{\mathbf{6}})+(\overline{\mathbf{6}}, \mathbf{6})]$} & $L L \bar{R} \bar{R}+R R \bar{L} \bar{L}$ \\
\hline
\end{tabular}

There are altogether ten independent scalar currents as listed in the following:

$$
\begin{gathered}
\eta_{1, N}^{\mathrm{S}, \mathbb{O}}=\lambda_{N}^{D B}\left(q_{A}^{a T} \mathbb{C} \gamma_{5} q_{B}^{b}\right)\left(\bar{q}_{A}^{a} \gamma_{5} \mathbb{C} \bar{q}_{D}^{b T}-\bar{q}_{A}^{b} \gamma_{5} \mathbb{C} \bar{q}_{D}^{a T}\right), \\
\eta_{2, N}^{\mathrm{S}, \mathbb{Q}}=\lambda_{N}^{D B}\left(q_{A}^{a T} \mathbb{C} q_{B}^{b}\right)\left(\bar{q}_{A}^{a} \mathbb{C} \bar{q}_{D}^{b T}-\bar{q}_{A}^{b} \mathbb{C} \bar{q}_{D}^{a T}\right), \\
\eta_{3, N}^{\mathrm{S}, \mathbb{Q}}=\lambda_{N}^{D B}\left(q_{A}^{a T} \mathbb{C} \sigma_{\mu \nu} q_{B}^{b}\right)\left(\bar{q}_{A}^{a} \sigma^{\mu \nu} \mathbb{C} \bar{q}_{D}^{b T}+\bar{q}_{A}^{b} \sigma^{\mu \nu} \mathbb{C} \bar{q}_{D}^{a T}\right), \\
\eta_{4, N}^{\mathrm{S}, \mathbb{O}}=\lambda_{N}^{D B}\left(q_{A}^{a T} \mathbb{C} \gamma_{5} q_{B}^{b}\right)\left(\bar{q}_{A}^{a} \gamma_{5} \mathbb{C} \bar{q}_{D}^{b T}+\bar{q}_{A}^{b} \gamma_{5} \mathbb{C} \bar{q}_{D}^{a T}\right), \\
\eta_{5, N}^{\mathrm{S}, \mathbb{Q}}=\lambda_{N}^{D B}\left(q_{A}^{a T} \mathbb{C} q_{B}^{b}\right)\left(\bar{q}_{A}^{a} \mathbb{C} \bar{q}_{D}^{b T}+\bar{q}_{A}^{b} \mathbb{C} \bar{q}_{D}^{a T}\right), \\
\eta_{6, N}^{\mathrm{S}, \mathbb{Q}}=\lambda_{N}^{D B}\left(q_{A}^{a T} \mathbb{C} \sigma_{\mu \nu} q_{B}^{b}\right)\left(\bar{q}_{A}^{a} \sigma^{\mu \nu} \mathbb{C} \bar{q}_{D}^{b T}-\bar{q}_{A}^{b} \sigma^{\mu \nu} \mathbb{C} \bar{q}_{D}^{a T}\right), \\
\eta_{7, N}^{\mathrm{S}, \mathbb{O}}=\lambda_{N}^{D B}\left(q_{A}^{a T} \mathbb{C} \gamma_{\mu} \gamma_{5} q_{B}^{b}\right)\left(\bar{q}_{A}^{a} \gamma^{\mu} \gamma_{5} \mathbb{C} \bar{q}_{D}^{b T}-\bar{q}_{A}^{b} \gamma^{\mu} \gamma_{5} \mathbb{C} \bar{q}_{D}^{a T}\right), \\
\eta_{8, N}^{\mathrm{S}, \mathbb{O}}=\lambda_{N}^{D B}\left(q_{A}^{a T} \mathbb{C} \gamma_{\mu} q_{B}^{b}\right)\left(\bar{q}_{A}^{a} \gamma^{\mu} \mathbb{C} \bar{q}_{D}^{b T}+\bar{q}_{A}^{b} \gamma^{\mu} \mathbb{C} \bar{q}_{D}^{a T}\right), \\
\eta_{9, N}^{\mathrm{S}, \mathbb{O}}=\lambda_{N}^{D B}\left(q_{A}^{a T} \mathbb{C} \gamma_{\mu} \gamma_{5} q_{B}^{b}\right)\left(\bar{q}_{A}^{a} \gamma^{\mu} \gamma_{5} \mathbb{C} \bar{q}_{D}^{b T}+\bar{q}_{A}^{b} \gamma^{\mu} \gamma_{5}^{\mathbb{C}} \bar{q}_{D}^{a T}\right), \\
\eta_{10, N}^{\mathrm{S}, \mathbb{O}}=\lambda_{N}^{D B}\left(q_{A}^{a T} \mathbb{C} \gamma_{\mu} q_{B}^{b}\right)\left(\bar{q}_{A}^{a} \gamma^{\mu} \mathbb{C} \bar{q}_{D}^{b T}-\bar{q}_{A}^{b} \gamma^{\mu} \mathbb{C} \bar{q}_{D}^{a T}\right) .
\end{gathered}
$$

The two superscripts $S$ and $\mathbb{O}$ denote scalar and flavor octet, respectively. Five currents $\eta_{1,2,3,7,8}^{\mathrm{S}, \mathbb{O}}$ contain diquarks and antidiquarks having the antisymmetric flavor structure $\overline{\mathbf{3}} \otimes \mathbf{3}$ and other five currents $\eta_{4,5,6,9,10}^{\mathrm{S}, \mathbb{O}}$ contain diquarks and antidiquarks having the symmetric flavor structure $\mathbf{6} \otimes \overline{\mathbf{6}}$. From the following combinations we can clearly see their chiral structure, where the left handed quark field $L_{A}^{a} \equiv q_{L A}^{a}=$ $\left(\left(1-\gamma_{5}\right) / 2\right) q_{A}^{a}$ and the right handed quark field $R_{A}^{a} \equiv q_{R A}^{a}=$ $\left(\left(1+\gamma_{5}\right) / 2\right) q_{A}^{a}$ are used:

$$
\begin{aligned}
\eta_{1, N}^{\mathrm{S}, \mathbb{Q}}-\eta_{2, N}^{\mathrm{S}, \mathbb{Q}}= & -2 \lambda_{N}^{D B} L_{A}^{a T} \mathbb{C} L_{B}^{b}\left(\bar{L}_{A}^{a} \mathbb{C} \bar{L}_{D}^{b T}-\bar{L}_{A}^{b} \mathbb{C} \bar{L}_{D}^{a T}\right) \\
& -2 \lambda_{N}^{D B} R_{A}^{a T} \mathbb{C} R_{B}^{b}\left(\bar{R}_{A}^{a} \mathbb{C} \bar{R}_{D}^{b T}-\bar{R}_{A}^{b} \mathbb{C} \bar{R}_{D}^{a T}\right), \\
\eta_{1, N}^{\mathrm{S}, \mathbb{Q}}+\eta_{2, N}^{\mathrm{S}, \mathbb{Q}}= & 2 \lambda_{N}^{D B} L_{A}^{a T} \mathbb{C} L_{B}^{b}\left(\bar{R}_{A}^{a} \mathbb{C} \bar{R}_{D}^{b T}-\bar{R}_{A}^{b} \mathbb{C} \bar{R}_{D}^{a T}\right) \\
& +2 \lambda_{N}^{D B} R_{A}^{a T} \mathbb{C} R_{B}^{b}\left(\bar{L}_{A}^{a} \mathbb{C} \bar{L}_{D}^{b T}-\bar{L}_{A}^{b} \mathbb{C} \bar{L}_{D}^{a T}\right), \\
\eta_{4, N}^{\mathrm{S}, \mathbb{Q}}-\eta_{5, N}^{\mathrm{S}, \mathbb{Q}}= & -2 \lambda_{N}^{D B} L_{A}^{a T} \mathbb{C} L_{B}^{b}\left(\bar{L}_{A}^{a} \mathbb{C} \bar{L}_{D}^{b T}+\bar{L}_{A}^{b} \mathbb{C} \bar{L}_{D}^{a T}\right) \\
& -2 \lambda_{N}^{D B} R_{A}^{a T} \mathbb{C} R_{B}^{b}\left(\bar{R}_{A}^{a} \mathbb{C} \bar{R}_{D}^{b T}+\bar{R}_{A}^{b} \mathbb{C} \bar{R}_{D}^{a T}\right),
\end{aligned}
$$

$$
\begin{aligned}
& \eta_{4, N}^{\mathrm{S}, \mathbb{Q}}+\eta_{5, N}^{\mathrm{S}, \mathbb{O}}=2 \lambda_{N}^{D B} L_{A}^{a T} \mathbb{C} L_{B}^{b}\left(\bar{R}_{A}^{a} \mathbb{C} \bar{R}_{D}^{b T}+\bar{R}_{A}^{b} \mathbb{C} \bar{R}_{D}^{a T}\right) \\
& +2 \lambda_{N}^{D B} R_{A}^{a T} \mathbb{C} R_{B}^{b}\left(\bar{L}_{A}^{a} \mathbb{C} \bar{L}_{D}^{b T}+\bar{L}_{A}^{b} \mathbb{C} \bar{L}_{D}^{a T}\right), \\
& \eta_{3, N}^{\mathrm{S}, \mathbb{Q}}=\lambda_{N}^{D B} L_{A}^{a T} \mathbb{C} \sigma_{\mu \nu} L_{B}^{b} \\
& \times\left(\bar{R}_{A}^{a} \sigma_{\mu \nu} \mathbb{C} \bar{R}_{D}^{b T}+\bar{R}_{A}^{b} \sigma_{\mu \nu} \mathbb{C} \bar{R}_{D}^{a T}\right) \\
& +\lambda_{N}^{D B} R_{A}^{a T} \mathbb{C} \sigma_{\mu \nu} R_{B}^{b} \\
& \times\left(\bar{L}_{A}^{a} \sigma_{\mu \nu} \mathbb{C} \bar{L}_{D}^{b T}+\bar{L}_{A}^{b} \sigma_{\mu \nu} \mathbb{C} \bar{L}_{D}^{a T}\right), \\
& \eta_{6, N}^{\mathrm{S}, \mathbb{Q}}=\lambda_{N}^{D B} L_{A}^{a T} \mathbb{C} \sigma_{\mu \nu} L_{B}^{b} \\
& \times\left(\bar{R}_{A}^{a} \sigma_{\mu \nu} \mathbb{C} \bar{R}_{D}^{b T}-\bar{R}_{A}^{b} \sigma_{\mu \nu} \mathbb{C} \bar{R}_{D}^{a T}\right) \\
& +\lambda_{N}^{D B} R_{A}^{a T} \mathbb{C} \sigma_{\mu \nu} R_{B}^{b} \\
& \times\left(\bar{L}_{A}^{a} \sigma_{\mu \nu} \mathbb{C} \bar{L}_{D}^{b T}-\bar{L}_{A}^{b} \sigma_{\mu \nu} \mathbb{C} \bar{L}_{D}^{a T}\right), \\
& \eta_{7, N}^{\mathrm{S}, \mathbb{Q}}-\eta_{10, N}^{\mathrm{S}, \mathbb{O}}=-2 \lambda_{N}^{D B} L_{A}^{a T} \mathbb{C} \gamma_{\mu} R_{B}^{b} \\
& \times\left(\bar{L}_{A}^{a} \gamma^{\mu} \mathbb{C} \bar{R}_{D}^{b T}-\bar{L}_{A}^{b} \gamma^{\mu} \mathbb{C} \bar{R}_{D}^{a T}\right) \\
& -2 \lambda_{N}^{D B} R_{A}^{a T} \mathbb{C} \gamma_{\mu} L_{B}^{b} \\
& \times\left(\bar{R}_{A}^{a} \gamma^{\mu} \mathbb{C} \bar{L}_{D}^{b T}-\bar{R}_{A}^{b} \gamma^{\mu} \mathbb{C} \bar{L}_{D}^{a T}\right), \\
& 5 \eta_{7, N}^{\mathrm{S}, \mathbb{Q}}+\eta_{10, N}^{\mathrm{S}, \mathbb{Q}}=6 d^{N M O} \lambda_{M}^{C A} \lambda_{O}^{D B} L_{A}^{a T} \mathbb{C} \gamma_{\mu} R_{B}^{b} \\
& \times\left(\bar{L}_{C}^{a} \gamma^{\mu} \mathbb{C} \bar{R}_{D}^{b T}-\bar{L}_{C}^{b} \gamma^{\mu} \mathbb{C} \bar{R}_{D}^{a T}\right), \\
& \eta_{8, N}^{\mathrm{S}, \mathbb{O}}-\eta_{9, N}^{\mathrm{S}, \mathbb{O}}=2 \lambda_{N}^{D B} L_{A}^{a T} \mathbb{C} \gamma_{\mu} R_{B}^{b} \\
& \times\left(\bar{L}_{A}^{a} \gamma^{\mu} \mathbb{C} \bar{R}_{D}^{b T}+\bar{L}_{A}^{b} \gamma^{\mu} \mathbb{C} \bar{R}_{D}^{a T}\right) \\
& +2 \lambda_{N}^{D B} R_{A}^{a T} \mathbb{C} \gamma_{\mu} L_{B}^{b} \\
& \times\left(\bar{R}_{A}^{a} \gamma^{\mu} \mathbb{C} \bar{L}_{D}^{b T}+\bar{R}_{A}^{b} \gamma^{\mu} \mathbb{C} \bar{L}_{D}^{a T}\right), \\
& 5 \eta_{8, N}^{\mathrm{S}, \mathbb{O}}+\eta_{9, N}^{\mathrm{S}, \mathbb{O}}=-6 d^{N M O} \lambda_{M}^{C A} \lambda_{O}^{D B} L_{A}^{a T} \mathbb{C} \gamma_{\mu} R_{B}^{b} \\
& \times\left(\bar{L}_{C}^{a} \gamma^{\mu} \mathbb{C} \bar{R}_{D}^{b T}+\bar{L}_{C}^{b} \gamma^{\mu} \mathbb{C} \bar{R}_{D}^{a T}\right) .
\end{aligned}
$$

We list their chirality and chiral representations in Table 4.

A.3. Pseudoscalar Tetraquark Currents of Flavor Octet. In this subsection we study flavor octet tetraquark currents of $J^{P}=0^{-}$. There are altogether ten independent pseudoscalar currents as listed in the following: 
TABLE 4: Flavor octet tetraquark currents of $J^{P}=0^{+}$classified in Appendix A.2.

\begin{tabular}{|c|c|c|}
\hline $\begin{array}{l}\text { Tetraquark currents of } \\
\left(\mathbf{8}_{F}, J^{P}=0^{+}\right)\end{array}$ & $\begin{array}{l}\text { Chiral } \\
\text { representations }\end{array}$ & Chirality \\
\hline$\eta_{1, N}^{\mathrm{S}, \mathbb{O}}-\eta_{2, N}^{\mathrm{S}, \mathbb{O}}, \eta_{4, N}^{\mathrm{S}, \mathbb{O}}-\eta_{5, N}^{\mathrm{S}, \mathbb{O}}$ & {$[(\mathbf{8}, \mathbf{1})+(\mathbf{1}, \mathbf{8})]$} & $L L \bar{L} \bar{L}+R R \bar{R} \bar{R}$ \\
\hline$\eta_{1, N}^{\mathrm{S}, \mathbb{Q}}+\eta_{2, N}^{\mathrm{S}, \mathbb{Q}}$ & {$[(\overline{3}, 3)+(3, \overline{3})]$} & $L L \bar{R} \bar{R}+R R \bar{L} \bar{L}$ \\
\hline$\eta_{4, N}^{\mathrm{S}, \mathrm{O}}+\eta_{5, N}^{\mathrm{S}, \mathrm{O}}$ & {$[(\mathbf{6}, \overline{\mathbf{6}})+(\overline{\mathbf{6}}, \mathbf{6})]$} & $L L \bar{R} \bar{R}+R R \bar{L} \bar{L}$ \\
\hline$\eta_{3, N}^{S, O}$ & {$[(\overline{\mathbf{3}}, \mathbf{3})+(\mathbf{3}, \overline{\mathbf{3}})]$} & $L L \bar{R} \bar{R}+R R \bar{L} \bar{L}$ \\
\hline$\eta_{6, N}^{\mathrm{s,O}, \mathrm{O}}$ & {$[(\mathbf{6}, \overline{\mathbf{6}})+(\overline{\mathbf{6}}, \mathbf{6})]$} & $L L \bar{R} \bar{R}+R R \bar{L} \bar{L}$ \\
\hline$\eta_{7, N}^{\mathrm{S}, \mathrm{O}}-\eta_{10, N}^{\mathrm{S}, \mathrm{O}}, \eta_{8, N}^{\mathrm{S}, \mathbb{O}}-\eta_{9, N}^{\mathrm{S}, \mathbb{O}}$ & {$[(\mathbf{1}, \mathbf{8})+(\mathbf{8}, \mathbf{1})]$} & $L R \bar{L} \bar{R}+R L \bar{R} \bar{L}$ \\
\hline $5 \eta_{7, N}^{\mathrm{S}, \mathbb{Q}}+\eta_{10, N}^{\mathrm{S}, \mathbb{O}}, 5 \eta_{8, N}^{\mathrm{S}, \mathbb{Q}}+\eta_{9, N}^{\mathrm{S}, \mathbb{Q}}$ & {$[(\mathbf{8}, \mathbf{8})+(\mathbf{8}, \mathbf{8})]$} & $L R \bar{L} \bar{R}+R L \bar{R} \bar{L}$ \\
\hline
\end{tabular}

$$
\begin{gathered}
\eta_{1, N}^{\mathrm{PS}, \mathbb{Q}}=\lambda_{N}^{B D}\left(q_{A}^{a T} \mathbb{C} q_{B}^{b}\right)\left(\bar{q}_{A}^{a} \gamma_{5} \mathbb{C} \bar{q}_{D}^{b T}-\bar{q}_{A}^{b} \gamma_{5} \mathbb{C} \bar{q}_{D}^{a T}\right), \\
\eta_{2, N}^{\mathrm{PS}, \mathbb{O}}=\lambda_{N}^{B D}\left(q_{A}^{a T} \mathbb{C} \gamma_{5} q_{B}^{b}\right)\left(\bar{q}_{A}^{a} \mathbb{C} \bar{q}_{D}^{b T}-\bar{q}_{A}^{b} \mathbb{C} \bar{q}_{D}^{a T}\right), \\
\eta_{3, N}^{\mathrm{PS}, \mathbb{O}}=\lambda_{N}^{B D}\left(q_{A}^{a T} \mathbb{C} \sigma_{\mu \nu} q_{B}^{b}\right)\left(\bar{q}_{A}^{a} \sigma^{\mu \nu} \gamma_{5} \mathbb{C} \bar{q}_{D}^{b T}+\bar{q}_{A}^{b} \sigma^{\mu \nu} \gamma_{5} \mathbb{C} \bar{q}_{D}^{a T}\right), \\
\eta_{4, N}^{\mathrm{PS}, \mathbb{O}}=\lambda_{N}^{B D}\left(q_{A}^{a T} \mathbb{C} q_{B}^{b}\right)\left(\bar{q}_{A}^{a} \gamma_{5} \mathbb{C} \bar{q}_{D}^{b T}+\bar{q}_{A}^{b} \gamma_{5} \mathbb{C} \bar{q}_{D}^{a T}\right), \\
\eta_{5, N}^{\mathrm{PS}, \mathbb{O}}=\lambda_{N}^{B D}\left(q_{A}^{a T} \mathbb{C} \gamma_{5} q_{B}^{b}\right)\left(\bar{q}_{A}^{a} \mathbb{C} \bar{q}_{D}^{b T}+\bar{q}_{A}^{b} \mathbb{C} \bar{q}_{D}^{a T}\right), \\
\eta_{6, N}^{\mathrm{PS}, \mathbb{O}}=\lambda_{N}^{B D}\left(q_{A}^{a T} \mathbb{C} \sigma_{\mu \nu} q_{B}^{b}\right)\left(\bar{q}_{A}^{a} \sigma^{\mu \nu} \gamma_{5} \mathbb{C} \bar{q}_{D}^{b T}-\bar{q}_{A}^{b} \sigma^{\mu \nu} \gamma_{5} \mathbb{C} \bar{q}_{D}^{a T}\right), \\
\eta_{7, N}^{\mathrm{PS}, \mathbb{Q}}=\lambda_{N}^{B D}\left(q_{A}^{a T} \mathbb{C} \gamma_{\mu} q_{B}^{b}\right)\left(\bar{q}_{A}^{a} \gamma^{\mu} \gamma_{5} \mathbb{C} \bar{q}_{D}^{b T}-\bar{q}_{A}^{b} \gamma^{\mu} \gamma_{5} \mathbb{C} \bar{q}_{D}^{a T}\right), \\
\eta_{8, N}^{\mathrm{PS}, \mathbb{O}}=\lambda_{N}^{B D}\left(q_{A}^{a T} \mathbb{C} \gamma_{\mu} \gamma_{5} q_{B}^{b}\right)\left(\bar{q}_{A}^{a} \gamma^{\mu} \mathbb{C} \bar{q}_{D}^{b T}+\bar{q}_{A}^{b} \gamma^{\mu} \mathbb{C} \bar{q}_{D}^{a T}\right), \\
\eta_{9, N}^{\mathrm{PS}, \mathbb{O}}=\lambda_{N}^{B D}\left(q_{A}^{a T} \mathbb{C} \gamma_{\mu} q_{B}^{b}\right)\left(\bar{q}_{A}^{a} \gamma^{\mu} \gamma_{5} \mathbb{C} \bar{q}_{D}^{b T}+\bar{q}_{A}^{b} \gamma^{\mu} \gamma_{5} \mathbb{C} \bar{q}_{D}^{a T}\right), \\
\eta_{10, N}^{\mathrm{PS}, \mathbb{O}}=\lambda_{N}^{B D}\left(q_{A}^{a T} \mathbb{C} \gamma_{\mu} \gamma_{5} q_{B}^{b}\right)\left(\bar{q}_{A}^{a} \gamma^{\mu} \mathbb{C} \bar{q}_{D}^{b T}-\bar{q}_{A}^{b} \gamma^{\mu} \mathbb{C} \bar{q}_{D}^{a T}\right) .
\end{gathered}
$$

The two superscripts PS and (I) denote pseudoscalar and flavor octet, respectively. Among these ten currents, $\eta_{1,2,3}^{\mathrm{PS}, \mathbb{O}}$ contain diquarks and antidiquarks having both the antisymmetric flavor structure $\overline{\mathbf{3}} \otimes 3 ; \eta_{4,5,6}^{\mathrm{PS}, \mathbb{Q}}$ contain diquarks and antidiquarks having both the symmetric flavor structure $\mathbf{6} \otimes \overline{\mathbf{6}} ; \eta_{7,8}^{\mathrm{PS}, \mathbb{O}}$ contain diquarks having the symmetric flavor structure and antidiquarks the antisymmetric flavor structure $6 \otimes 3 ; \eta_{9,10}^{\mathrm{PS}, \mathbb{Q}}$ contain diquarks having the antisymmetric flavor structure and antidiquarks the symmetric flavor structure $\overline{\mathbf{3}} \otimes \overline{\mathbf{6}}$. From the following combinations we can clearly see their chiral structure, where the left handed quark field $L_{A}^{a} \equiv$ $q_{L A}^{a}=\left(\left(1-\gamma_{5}\right) / 2\right) q_{A}^{a}$ and the right handed quark field $R_{A}^{a} \equiv$ $q_{R A}^{a}=\left(\left(1+\gamma_{5}\right) / 2\right) q_{A}^{a}$ are used:

$$
\begin{aligned}
\eta_{1, N}^{\mathrm{PS}, \mathbb{Q}}-\eta_{2, N}^{\mathrm{PS}, \mathbb{Q}}= & 2 \lambda_{N}^{D B} L_{A}^{a T} \mathbb{C} L_{B}^{b}\left(\bar{L}_{A}^{a} \mathbb{C} \bar{L}_{D}^{b T}-\bar{L}_{A}^{b} \mathbb{C} \bar{L}_{D}^{a T}\right) \\
& -2 \lambda_{N}^{D B} R_{A}^{a T} \mathbb{C} R_{B}^{b}\left(\bar{R}_{A}^{a} \mathbb{C} \bar{R}_{D}^{b T}-\bar{R}_{A}^{b} \mathbb{C} \bar{R}_{D}^{a T}\right),
\end{aligned}
$$

$$
\begin{aligned}
& \eta_{1, N}^{\mathrm{PS}, \mathbb{O}}+\eta_{2, N}^{\mathrm{PS}, \mathbb{O}}=-2 \lambda_{N}^{D B} L_{A}^{a T} \mathbb{C} L_{B}^{b}\left(\bar{R}_{A}^{a} \mathbb{C} \bar{R}_{D}^{b T}-\bar{R}_{A}^{b} \mathbb{C} \bar{R}_{D}^{a T}\right) \\
& +2 \lambda_{N}^{D B} R_{A}^{a T} \mathbb{C} R_{B}^{b}\left(\bar{L}_{A}^{a} \mathbb{C} \bar{L}_{D}^{b T}-\bar{L}_{A}^{b} \mathbb{C} \bar{L}_{D}^{a T}\right), \\
& \eta_{4, N}^{\mathrm{PS}, \mathbb{Q}}-\eta_{5, N}^{\mathrm{PS}, \mathbb{Q}}=2 \lambda_{N}^{D B} L_{A}^{a T} \mathbb{C} L_{B}^{b}\left(\bar{L}_{A}^{a} \mathbb{C} \bar{L}_{D}^{b T}+\bar{L}_{A}^{b} \mathbb{C} \bar{L}_{D}^{a T}\right) \\
& -2 \lambda_{N}^{D B} R_{A}^{a T} \mathbb{C} R_{B}^{b}\left(\bar{R}_{A}^{a} \mathbb{C} \bar{R}_{D}^{b T}+\bar{R}_{A}^{b} \mathbb{C} \bar{R}_{D}^{a T}\right), \\
& \eta_{4, N}^{\mathrm{PS}, \mathbb{O}}+\eta_{5, N}^{\mathrm{PS}, \mathbb{O}}=-2 \lambda_{N}^{D B} L_{A}^{a T} \mathbb{C} L_{B}^{b}\left(\bar{R}_{A}^{a} \mathbb{C} \bar{R}_{D}^{b T}+\bar{R}_{A}^{b} \mathbb{C} \bar{R}_{D}^{a T}\right) \\
& +2 \lambda_{N}^{D B} R_{A}^{a T} \mathbb{C} R_{B}^{b}\left(\bar{L}_{A}^{a} \mathbb{C} \bar{L}_{D}^{b T}+\bar{L}_{A}^{b} \mathbb{C} \bar{L}_{D}^{a T}\right), \\
& \eta_{3, \mathrm{~N}}^{\mathrm{PS}, \mathbb{O}}=-\lambda_{N}^{D B} L_{A}^{a T} \mathbb{C} \sigma_{\mu \nu} L_{B}^{b} \\
& \times\left(\bar{R}_{A}^{a} \sigma_{\mu \nu} \mathbb{C} \bar{R}_{D}^{b T}+\bar{R}_{A}^{b} \sigma_{\mu \nu} \mathbb{C} \bar{R}_{D}^{a T}\right) \\
& +\lambda_{N}^{D B} R_{A}^{a T} \mathbb{C} \sigma_{\mu \nu} R_{B}^{b} \\
& \times\left(\bar{L}_{A}^{a} \sigma_{\mu \nu} \mathbb{C} \bar{L}_{D}^{b T}+\bar{L}_{A}^{b} \sigma_{\mu \nu} \mathbb{C} \bar{L}_{D}^{a T}\right), \\
& \eta_{6, N}^{\mathrm{PS}, \mathbb{O}}=-\lambda_{N}^{D B} L_{A}^{a T} \mathbb{C} \sigma_{\mu \nu} L_{B}^{b} \\
& \times\left(\bar{R}_{A}^{a} \sigma_{\mu \nu} \mathbb{C} \bar{R}_{D}^{b T}-\bar{R}_{A}^{b} \sigma_{\mu \nu} \mathbb{C} \bar{R}_{D}^{a T}\right) \\
& +\lambda_{N}^{D B} R_{A}^{a T} \mathbb{C} \sigma_{\mu \nu} R_{B}^{b} \\
& \times\left(\bar{L}_{A}^{a} \sigma_{\mu \nu} \mathbb{C} \bar{L}_{D}^{b T}-\bar{L}_{A}^{b} \sigma_{\mu \nu} \mathbb{C} \bar{L}_{D}^{a T}\right), \\
& \eta_{8, N}^{\mathrm{PS}, \mathbb{O}}-\eta_{9, N}^{\mathrm{PS}, \mathbb{O}}=2 \lambda_{N}^{D B} L_{A}^{a T} \mathbb{C} \gamma_{\mu} R_{B}^{b}\left(\bar{L}_{A}^{a} \gamma^{\mu} \mathbb{C}_{D}^{b T}+\bar{L}_{A}^{b} \gamma^{\mu} \mathbb{C} \bar{R}_{D}^{a T}\right) \\
& -2 \lambda_{N}^{D B} R_{A}^{a T} \mathbb{C} \gamma_{\mu} L_{B}^{b} \\
& \times\left(\bar{R}_{A}^{a} \gamma^{\mu} \mathbb{C} \bar{L}_{D}^{b T}+\bar{R}_{A}^{b} \gamma^{\mu} \mathbb{C} \bar{L}_{D}^{a T}\right), \\
& \eta_{8, N}^{\mathrm{PS}, \mathbb{Q}}+\eta_{9, N}^{\mathrm{PS}, \mathbb{Q}}=-2 i f^{N M O} \lambda_{M}^{C A} \lambda_{O}^{D B} L_{A}^{a T} \mathbb{C} \gamma_{\mu} R_{B}^{b} \\
& \times\left(\bar{L}_{C}^{a} \gamma^{\mu} \mathbb{C} \bar{R}_{D}^{b T}+\bar{L}_{C}^{b} \gamma^{\mu} \mathbb{C} \bar{R}_{D}^{a T}\right), \\
& \eta_{7, N}^{\mathrm{PS}, \mathbb{Q}}-\eta_{10, N}^{\mathrm{PS}, \mathbb{Q}}=-2 \lambda_{N}^{D B} L_{A}^{a T} \mathbb{C} \gamma_{\mu} R_{B}^{b} \\
& \times\left(\bar{L}_{A}^{a} \gamma^{\mu} \mathbb{C} \bar{R}_{D}^{b T}-\bar{L}_{A}^{b} \gamma^{\mu} \mathbb{C} \bar{R}_{D}^{a T}\right) \\
& +2 \lambda_{N}^{D B} R_{A}^{a T} \mathbb{C} \gamma_{\mu} L_{B}^{b} \\
& \times\left(\bar{R}_{A}^{a} \gamma^{\mu} \mathbb{C} \bar{L}_{D}^{b T}-\bar{R}_{A}^{b} \gamma^{\mu} \mathbb{C} \bar{L}_{D}^{a T}\right), \\
& \eta_{7, N}^{\mathrm{PS}, \mathbb{O}}+\eta_{10, N}^{\mathrm{PS}, \mathbb{O}}=2 i f^{N M O} \lambda_{M}^{C A} \lambda_{O}^{D B} L_{A}^{a T} \mathbb{C} \gamma_{\mu} R_{B}^{b} \\
& \times\left(\bar{L}_{C}^{a} \gamma^{\mu} \mathbb{C} \bar{R}_{D}^{b T}-\bar{L}_{C}^{b} \gamma^{\mu} \mathbb{C} \bar{R}_{D}^{a T}\right) .
\end{aligned}
$$

We list their chirality and chiral representations in Table 5. 
TABLE 5: Flavor octet tetraquark currents of $J^{P}=0^{-}$classified in Appendix A.3.

\begin{tabular}{lcc}
\hline $\begin{array}{l}\text { Tetraquark currents of } \\
\left(\mathbf{8}_{F}, J^{P}=0^{-}\right)\end{array}$ & $\begin{array}{c}\text { Chiral } \\
\text { representations }\end{array}$ & Chirality \\
\hline$\eta_{1, N}^{\mathrm{PS}, \mathbb{O}}-\eta_{2, N}^{\mathrm{PS}, \mathbb{O}}, \eta_{4}^{\mathrm{PS}, \mathbb{O}}-\eta_{5}^{\mathrm{PS}, \mathbb{O}}$ & {$[(\mathbf{8}, \mathbf{1})+(\mathbf{1}, \mathbf{8})]$} & $L L \bar{L} \bar{L}+R R \bar{R} \bar{R}$ \\
$\eta_{1, N}^{\mathrm{PS}, \mathbb{O}}+\eta_{2, N}^{\mathrm{PS}, \mathbb{O}}$ & {$[(\overline{\mathbf{3}}, \mathbf{3})+(\mathbf{3}, \overline{\mathbf{3}})]$} & $L L \bar{R} \bar{R}+R R \bar{L} \bar{L}$ \\
$\eta_{4, N}^{\mathrm{PS}, \mathbb{O}}+\eta_{5, N}^{\mathrm{PS}, \mathbb{O}}$ & {$[(\mathbf{6}, \overline{\mathbf{6}})+(\overline{\mathbf{6}}, \mathbf{6})]$} & $L L \bar{R} \bar{R}+R R \bar{L} \bar{L}$ \\
$\eta_{3, N}^{\mathrm{PS}, \mathbb{O}}$ & {$[(\overline{\mathbf{3}}, \mathbf{3})+(\mathbf{3}, \overline{\mathbf{3}})]$} & $L L \bar{R} \bar{R}+R R \bar{L} \bar{L}$ \\
$\eta_{6, N}^{\mathrm{PS}, \mathbb{O}}$ & {$[(\mathbf{6}, \overline{\mathbf{6}})+(\overline{\mathbf{6}}, \mathbf{6})]$} & $L L \bar{R} \bar{R}+R R \bar{L} \bar{L}$ \\
$\eta_{8, N}^{\mathrm{PS}, \mathbb{O}}-\eta_{9, N}^{\mathrm{PS}, \mathbb{O}}, \eta_{7, N}^{\mathrm{PS}, \mathbb{O}}-\eta_{10, N}^{\mathrm{PS}, \mathbb{O}}$ & {$[(\mathbf{1}, \mathbf{8})+(\mathbf{8}, \mathbf{1})]$} & $L R \bar{L} \bar{R}+R L \bar{R} \bar{L}$ \\
$\eta_{8, N}^{\mathrm{PS}, \mathbb{O}}+\eta_{9, N}^{\mathrm{PS} \mathbb{O}}, \eta_{7, N}^{\mathrm{PS}, \mathbb{O}}+\eta_{10, N}^{\mathrm{PS} \mathbb{O}}$ & {$[(\mathbf{8}, \mathbf{8})+(\mathbf{8}, \mathbf{8})]$} & $L R \bar{L} \bar{R}+R L \bar{R} \bar{L}$ \\
\hline
\end{tabular}

A.4. Scalar Tetraquark Currents of Flavor $27_{F}$. In this subsection we study flavor $27_{F}$ tetraquark currents of $J^{P}=0^{+}$. There are altogether five independent scalar currents as listed in the following:

$$
\begin{gathered}
\eta_{1, U}^{\mathrm{S}, \mathbb{S}}=S_{U}^{C D ; A B}\left(q_{A}^{a T} C \gamma_{5} q_{B}^{b}\right)\left(\bar{q}_{C}^{a} \gamma_{5} C \bar{q}_{D}^{b T}\right), \\
\eta_{2, U}^{\mathrm{S}, \mathbb{S}}=S_{U}^{C D ; A B}\left(q_{A}^{a T} C q_{B}^{b}\right)\left(\bar{q}_{C}^{a} C \bar{q}_{D}^{b T}\right), \\
\eta_{3, U}^{\mathrm{S}, \mathbb{S} S}=S_{U}^{C D ; A B}\left(q_{A}^{a T} C \sigma_{\mu \nu} q_{B}^{b}\right)\left(\bar{q}_{C}^{a} \sigma^{\mu \nu} C \bar{q}_{D}^{b T}\right), \\
\eta_{4, U}^{\mathrm{S}, \mathbb{S}}=S_{U}^{C D ; A B}\left(q_{A}^{a T} C \gamma_{\mu} \gamma_{5} q_{B}^{b}\right)\left(\bar{q}_{C}^{a} \gamma^{\mu} \gamma_{5} C \bar{q}_{D}^{b T}\right), \\
\eta_{5, U}^{\mathrm{S}, \mathbb{S} S}=S_{U}^{C D ; A B}\left(q_{A}^{a T} C \gamma_{\mu} q_{B}^{b}\right)\left(\bar{q}_{C}^{a} \gamma^{\mu} C \bar{q}_{D}^{b T}\right) .
\end{gathered}
$$

All these five currents contain diquarks and antidiquarks having the symmetric flavor structure $\mathbf{6} \otimes \overline{\mathbf{6}}$. From the following combinations we can clearly see their chiral structure, where the left handed quark field $L_{A}^{a} \equiv q_{L A}^{a}=\left(\left(1-\gamma_{5}\right) / 2\right) q_{A}^{a}$ and the right handed quark field $R_{A}^{a} \equiv q_{R A}^{a}=\left(\left(1+\gamma_{5}\right) / 2\right) q_{A}^{a}$ are used:

$$
\begin{aligned}
& \eta_{1, U}^{\mathrm{S}, \mathbb{W S}}-\eta_{2, U}^{\mathrm{S}, \mathbb{S S}}=-2 S_{U}^{C D ; A B} L_{A}^{a T} C L_{B}^{b} \bar{L}_{C}^{a} C \bar{L}_{D}^{b T} \\
& -2 S_{U}^{C D ; A B} R_{A}^{a T} C R_{B}^{b} \bar{R}_{C}^{a} C \bar{R}_{D}^{b T} \text {, } \\
& \eta_{1, U}^{\mathrm{S}, \mathbb{S}}+\eta_{2, U}^{\mathrm{S}, \mathbb{U S}}=2 S_{U}^{C D ; A B} L_{A}^{a T} C L_{B}^{b} \bar{R}_{C}^{a} C \bar{R}_{D}^{b T} \\
& +2 S_{U}^{C D ; A B} R_{A}^{a T} C R_{B}^{b} \bar{L}_{C}^{a} C \bar{L}_{D}^{b T}, \\
& \eta_{3, U}^{\mathrm{S}, \mathbb{S}}=S_{U}^{C D ; A B} L_{A}^{a T} C \sigma_{\mu \nu} L_{B}^{b} \bar{R}_{C}^{a} \sigma_{\mu \nu} C \bar{R}_{D}^{b T} \\
& +S_{U}^{C D ; A B} R_{A}^{a T} C \sigma_{\mu \nu} R_{B}^{b} \bar{L}_{C}^{a} \sigma_{\mu \nu} C \bar{L}_{D}^{b T}, \\
& \eta_{4, U}^{\mathrm{S}, \mathbb{S}}=-2 S_{U}^{C D ; A B} L_{A}^{a T} C \gamma_{\mu} R_{B}^{b} \\
& \times\left(\bar{L}_{C}^{a} \gamma^{\mu} C \bar{R}_{D}^{b T}+\bar{L}_{C}^{b} \gamma^{\mu} C \bar{R}_{D}^{a T}\right), \\
& \eta_{5, U}^{\mathrm{S}, \mathbb{T S}}=2 S_{U}^{C D ; A B} L_{A}^{a T} C \gamma_{\mu} R_{B}^{b}\left(\bar{L}_{C}^{a} \gamma^{\mu} C \bar{R}_{D}^{b T}-\bar{L}_{C}^{b} \gamma^{\mu} C \bar{R}_{D}^{a T}\right) .
\end{aligned}
$$

We list their chirality and chiral representations in Table 6.
TABLE 6: Flavor $27_{F}$ tetraquark currents of $J^{P}=0^{+}$classified in Appendix A.4.

\begin{tabular}{lcc}
\hline $\begin{array}{l}\text { Tetraquark currents of } \\
\left(\mathbf{2 7}, J^{P}=0^{+}\right)\end{array}$ & $\begin{array}{c}\text { Chiral } \\
\text { representations }\end{array}$ & Chirality \\
\hline$\eta_{1, U}^{\mathrm{S}, \mathbb{T S}}-\eta_{2, U}^{\mathrm{S}, \mathbb{T S}}$ & {$[(\mathbf{2 7}, \mathbf{1})+(\mathbf{1}, \mathbf{2 7})]$} & $L L \bar{L} \bar{L}+R R \bar{R} \bar{R}$ \\
$\eta_{1, U}^{\mathrm{S}, \mathbb{U S}}+\eta_{2, U}^{\mathrm{S}, \mathbb{S S}}$ & {$[(\mathbf{6}, \overline{\mathbf{6}})+(\overline{\mathbf{6}}, \mathbf{6})]$} & $L L \bar{R} \bar{R}+R R \bar{L} \bar{L}$ \\
$\eta_{3, U}^{\mathrm{S}, \mathbb{U S}}$ & {$[(\mathbf{6}, \overline{\mathbf{6}})+(\overline{\mathbf{6}}, \mathbf{6})]$} & $L L \bar{R} \bar{R}+R R \bar{L} \bar{L}$ \\
$\eta_{4, U}^{\mathrm{S} \mathbb{U S}}, \eta_{5, U}^{\mathrm{S}, \mathbb{S S}}$ & {$[(\mathbf{8}, \mathbf{8})+(\mathbf{8}, \mathbf{8})]$} & $L R \bar{L} \bar{R}+R L \bar{R} \bar{L}$ \\
\hline
\end{tabular}

TABLE 7: Flavor $27_{F}$ tetraquark currents of $J^{P}=0^{-}$classified in Appendix A.5.

\begin{tabular}{lcc}
\hline $\begin{array}{l}\text { Tetraquark currents of } \\
\left(\mathbf{2 7}_{F}, J^{P}=0^{-}\right)\end{array}$ & $\begin{array}{c}\text { Chiral } \\
\text { representations }\end{array}$ & Chirality \\
\hline$\eta_{1, U}^{\mathrm{PS}, \mathbb{S S}}-\eta_{2, U}^{\mathrm{PS}, \mathbb{T S}}$ & {$[(\mathbf{2 7}, \mathbf{1})+(1, \mathbf{2 7})]$} & $L L \bar{L} \bar{L}+R R \bar{R} \bar{R}$ \\
$\eta_{1, U}^{\mathrm{PS}, \mathbb{U S}}+\eta_{2, U}^{\mathrm{PS}, \mathbb{T S}}$ & {$[(\mathbf{6}, \overline{\mathbf{6}})+(\overline{\mathbf{6}}, \mathbf{6})]$} & $L L \bar{R} \bar{R}+R R \bar{L} \bar{L}$ \\
$\eta_{3, U}^{\mathrm{PS}, \mathbb{S S}}$ & {$[(\mathbf{6}, \overline{\mathbf{6}})+(\overline{\mathbf{6}}, \mathbf{6})]$} & $L L \bar{R} \bar{R}+R R \bar{L} \bar{L}$ \\
\hline
\end{tabular}

A.5. Pseudoscalar Tetraquark Currents of Flavor $27_{F}$. In this subsection we study flavor $27_{F}$ tetraquark currents of $J^{P}=0^{-}$. There are altogether three independent pseudoscalar currents as listed in the following:

$$
\begin{gathered}
\eta_{1, U}^{\mathrm{PS}, \mathbb{S S}}=S_{U}^{C D ; \mathrm{AB}}\left(q_{A}^{a T} C q_{B}^{b}\right)\left(\bar{q}_{C}^{a} \gamma_{5} C \bar{q}_{D}^{b T}\right), \\
\eta_{2, U}^{\mathrm{PS}, \mathbb{S}}=S_{U}^{C D ; A B}\left(q_{A}^{a T} C \gamma_{5} q_{B}^{b}\right)\left(\bar{q}_{C}^{a} C \bar{q}_{D}^{b T}\right), \\
\eta_{3, U}^{\mathrm{PS}, \mathbb{U S}}=S_{U}^{C D ; A B}\left(q_{A}^{a T} C \sigma_{\mu \nu} q_{B}^{b}\right)\left(\bar{q}_{C}^{a} \sigma^{\mu \nu} \gamma_{5} C \bar{q}_{D}^{b T}\right) .
\end{gathered}
$$

All these three currents contain diquarks and antidiquarks having the symmetric flavor structure $\mathbf{6} \otimes \overline{\mathbf{6}}$. From the following combinations we can clearly see their chiral structure, where the left handed quark field $L_{A}^{a} \equiv q_{L A}^{a}=\left(\left(1-\gamma_{5}\right) / 2\right) q_{A}^{a}$ and the right handed quark field $R_{A}^{a} \equiv q_{R A}^{a}=\left(\left(1+\gamma_{5}\right) / 2\right) q_{A}^{a}$ are used:

$$
\begin{aligned}
\eta_{1, U}^{\mathrm{PS}, \mathbb{U S}}-\eta_{2, U}^{\mathrm{PS}, \mathbb{U S}}= & 2 S_{U}^{C D ; A B} L_{A}^{a T} C L_{B}^{b} \bar{L}_{C}^{a} C \bar{L}_{D}^{b T} \\
& -2 S_{U}^{C D ; A B} R_{A}^{a T} C R_{B}^{b} \bar{R}_{C}^{a} C \bar{R}_{D}^{b T}, \\
\eta_{1, U}^{\mathrm{PS}, \mathbb{U S}}+\eta_{2, U}^{\mathrm{PS}, \mathbb{U S}}= & -2 S_{U}^{C D ; A B} L_{A}^{a T} C L_{B}^{b} \bar{R}_{C}^{a} C \bar{R}_{D}^{b T} \\
& +2 S_{U}^{C D ; A B} R_{A}^{a T} C R_{B}^{b} \bar{L}_{C}^{a} C \bar{L}_{D}^{b T}, \\
\eta_{3, U}^{\mathrm{PS}, \mathbb{U S}}= & -S_{U}^{C D ; A B} L_{A}^{a T} C \sigma_{\mu \nu} L_{B}^{b} \bar{R}_{C}^{a} \sigma_{\mu \nu} C \bar{R}_{D}^{b T} \\
& +S_{U}^{C D ; A B} R_{A}^{a T} C \sigma_{\mu \nu} R_{B}^{b} \bar{L}_{C}^{a} \sigma_{\mu \nu} C \bar{L}_{D}^{b T} .
\end{aligned}
$$

We list their chirality and chiral representations in Table 7. 
A.6. Pseudoscalar Tetraquark Currents of Flavor $\overline{\mathbf{1 0}}_{F}$. In this subsection we study flavor $\overline{\mathbf{1 0}}_{F}$ tetraquark currents of $J^{P}=0^{-}$. There are altogether two independent pseudoscalar currents as listed in the following:

$$
\begin{aligned}
& \eta_{1, P}^{\mathrm{PS}, \bar{D}}=\epsilon^{A B E} S_{P}^{C D E}\left(q_{A}^{a T} C \gamma_{\mu} q_{B}^{b}\right)\left(\bar{q}_{C}^{a} \gamma^{\mu} \gamma_{5} C \bar{q}_{D}^{b T}\right), \\
& \eta_{2, P}^{\mathrm{PS}, \overline{\mathbb{D}}}=\epsilon^{A B E} S_{P}^{C D E}\left(q_{A}^{a \mathrm{~T}} C \gamma_{\mu} \gamma_{5} q_{B}^{b}\right)\left(\bar{q}_{C}^{a} \gamma^{\mu} C \bar{q}_{D}^{b T}\right) .
\end{aligned}
$$

These two currents both contain diquarks having the antisymmetric flavor structure and antidiquarks the symmetric flavor structure $\overline{\mathbf{3}} \otimes \overline{\mathbf{6}}$. From the following combinations we can clearly see their chiral structure, where the left handed quark field $L_{A}^{a} \equiv q_{L A}^{a}=\left(\left(1-\gamma_{5}\right) / 2\right) q_{A}^{a}$ and the right handed quark field $R_{A}^{a} \equiv q_{R A}^{a}=\left(\left(1+\gamma_{5}\right) / 2\right) q_{A}^{a}$ are used:

$$
\begin{aligned}
\eta_{1, P}^{\mathrm{PS}, \overline{\mathbb{D}}}= & -2 \epsilon^{A B E} S_{P}^{C D E} L_{A}^{a T} C \gamma_{\mu} R_{B}^{b} \\
& \times\left(\bar{L}_{C}^{a} \gamma^{\mu} C \bar{R}_{D}^{b T}+\bar{L}_{C}^{b} \gamma^{\mu} C \bar{R}_{D}^{a T}\right), \\
\eta_{2, P}^{\mathrm{PS}, \overline{\mathbb{D}}}= & 2 \epsilon^{A B E} S_{P}^{C D E} L_{A}^{a T} C \gamma_{\mu} R_{B}^{b} \\
& \times\left(\bar{L}_{C}^{a} \gamma^{\mu} C \bar{R}_{D}^{b T}-\bar{L}_{C}^{b} \gamma^{\mu} C \bar{R}_{D}^{a T}\right) .
\end{aligned}
$$

We find that these two currents both belong to the chiral representation $[(\mathbf{8}, \mathbf{8})+(\mathbf{8}, \mathbf{8})]$ and their chirality is $L R \bar{L} \bar{R}+$ $R L \bar{R} \bar{L}$.

A.7. Pseudoscalar Tetraquark Currents of Flavor $\mathbf{1 0}_{F}$. In this subsection we study flavor $\mathbf{1 0}_{F}$ tetraquark currents of $J^{P}=0^{-}$. There are altogether two independent pseudoscalar currents as listed in the following:

$$
\begin{aligned}
& \eta_{1, P}^{\mathrm{PS}, \mathbb{D}}=S_{P}^{A B E} \epsilon^{C D E}\left(q_{A}^{a T} C \gamma_{\mu} q_{B}^{b}\right)\left(\bar{q}_{C}^{a} \gamma^{\mu} \gamma_{5} C \bar{q}_{D}^{b T}\right), \\
& \eta_{2, P}^{\mathrm{PS}, \mathbb{D}}=S_{P}^{A B E} \epsilon^{C D E}\left(q_{A}^{a T} C \gamma_{\mu} \gamma_{5} q_{B}^{b}\right)\left(\bar{q}_{C}^{a} \gamma^{\mu} C \bar{q}_{D}^{b T}\right) .
\end{aligned}
$$

These two currents both contain diquarks having the antisymmetric flavor structure and antidiquarks the symmetric flavor structure $\mathbf{6} \otimes 3$. From the following combinations we can clearly see their chiral structure, where the left handed quark field $L_{A}^{a} \equiv q_{L A}^{a}=\left(\left(1-\gamma_{5}\right) / 2\right) q_{A}^{a}$ and the right handed quark field $R_{A}^{a} \equiv q_{R A}^{a}=\left(\left(1+\gamma_{5}\right) / 2\right) q_{A}^{a}$ are used:

$$
\begin{aligned}
\eta_{1, P}^{\mathrm{PS}, \mathbb{D}}= & -2 S_{P}^{A B E} \epsilon{ }^{C D E} L_{A}^{a T} C \gamma_{\mu} R_{B}^{b} \\
& \times\left(\bar{L}_{C}^{a} \gamma^{\mu} C \bar{R}_{D}^{b T}-\bar{L}_{C}^{b} \gamma^{\mu} C \bar{R}_{D}^{a T}\right), \\
\eta_{2, P}^{\mathrm{PS}, \mathbb{D}}= & 2 S_{P}^{A B E} \epsilon^{C D E} L_{A}^{a T} C \gamma_{\mu} R_{B}^{b} \\
& \times\left(\bar{L}_{C}^{a} \gamma^{\mu} C \bar{R}_{D}^{b T}+\bar{L}_{C}^{b} \gamma^{\mu} C \bar{R}_{D}^{a T}\right) .
\end{aligned}
$$

We find that these two currents both belong to the chiral representation $[(\mathbf{8}, \mathbf{8})+(\mathbf{8}, \mathbf{8})]$ and their chirality is $L R \bar{L} \bar{R}+$ $R L \bar{R} \bar{L}$.

\section{B. Other Chiral Transformations}

There are four $[(\mathbf{1}, \mathbf{1})]$ chiral multiplets, $\left(\eta_{1}^{\mathrm{S}, \mathbb{S}}-\eta_{2}^{\mathrm{S}, \mathbb{S}}, \eta_{1}^{\mathrm{PS}, \mathbb{S}}-\right.$ $\left.\eta_{2}^{\mathrm{PS}, \mathbb{S}}\right),\left(\eta_{4}^{\mathrm{S}, \mathbb{S}}-\eta_{5}^{\mathrm{S}, \mathbb{S}}, \eta_{4}^{\mathrm{PS}, \mathbb{S}}-\eta_{5}^{\mathrm{PS}, \mathbb{S}}\right),\left(\eta_{7}^{\mathrm{S}, \mathbb{S}}-\eta_{10}^{\mathrm{S}, \mathbb{S}}, \eta_{7}^{\mathrm{PS}, \mathbb{S}}-\eta_{10}^{\mathrm{PS}, \mathbb{S}}=0\right)$, and $\left(\eta_{8}^{\mathrm{S}, \mathbb{S}}-\eta_{9}^{\mathrm{S}, \mathbb{S}}, \eta_{8}^{\mathrm{PS}, \mathbb{S}}-\eta_{9}^{\mathrm{PS}, \mathbb{S}}=0\right)$. We use $\left(\eta_{(\mathbf{1}, \mathbf{1})}^{\mathrm{S}, \mathbb{S}}, \eta_{(\mathbf{1}, \mathbf{1})}^{\mathrm{PS}, \mathbb{S}}\right)$ to denote them, and their chiral transformation properties are

$$
\begin{aligned}
& \delta_{5} \eta_{(\mathbf{1}, \mathbf{1})}^{\mathrm{S}(\mathrm{PS}), \mathbb{S}}=0, \\
& \delta^{\vec{a}} \eta_{(\mathbf{1}, \mathbf{1})}^{\mathrm{S}(\mathrm{PS}), \mathbb{S}}=0, \\
& \delta_{5}^{\vec{b}} \eta_{(\mathbf{1}, \mathbf{1})}^{\mathrm{S}(\mathrm{PS}) \mathbb{S}}=0 .
\end{aligned}
$$

There are two $[(\mathbf{6}, \overline{\mathbf{6}}) \oplus(\overline{\mathbf{6}}, \mathbf{6})]$ chiral multiplets, $\left(\eta_{4}^{\mathrm{S}, \mathbb{S}}+\eta_{5}^{\mathrm{S}, \mathbb{S}}, \eta_{4}^{\mathrm{PS}, \mathbb{S}}+\eta_{5}^{\mathrm{PS}, \mathbb{S}}, \eta_{4, N}^{\mathrm{S}, \mathbb{Q}}+\eta_{5, N}^{\mathrm{S}, \mathbb{O}}, \eta_{4, N}^{\mathrm{PS}, \mathbb{Q}}+\eta_{5, N}^{\mathrm{PS}, \mathbb{O}}, \eta_{1, U}^{\mathrm{S}, \mathbb{U S}}+\right.$ $\left.\eta_{2, U}^{\mathrm{S}, \mathbb{T S}}, \eta_{1, U}^{\mathrm{PS}, \mathbb{T S}}+\eta_{2, U}^{\mathrm{PS}, \mathbb{T S}}\right),\left(\eta_{6}^{\mathrm{S}, \mathbb{S}}, \eta_{6}^{\mathrm{PS}, \mathbb{S}}, \eta_{6, N}^{\mathrm{S}, \mathbb{O}}, \eta_{6, N}^{\mathrm{PS}, \mathbb{O}}, \eta_{3, U}^{\mathrm{S}, \mathbb{S S}}, \eta_{3, U}^{\mathrm{PS}, \mathbb{U S}}\right) . \mathrm{We}$ use $\left(\eta_{(\mathbf{6}, \overline{\mathbf{6}})}^{\mathrm{S} S}, \eta_{(\mathbf{6}, \overline{\mathbf{6}})}^{\mathrm{PS} S}, \eta_{(\mathbf{6}, \overline{\mathbf{6}}), N}^{\mathrm{S}, \mathbb{O}}, \eta_{(\mathbf{6}, \overline{\mathbf{6}}), N}^{\mathrm{PS}, \mathbb{O}}, \eta_{(\mathbf{6}, \overline{\mathbf{6}})}^{\mathrm{S}, \mathbb{S}}, \eta_{(\mathbf{6}, \overline{\mathbf{6}})}^{\mathrm{PS}, \mathbb{S}}\right)$ to denote them, and their chiral transformation properties are

$$
\begin{gathered}
\delta_{5} \eta_{(\mathbf{6}, \overline{\mathbf{6}})}^{\mathrm{S}(\mathrm{PS}), \mathbb{S}}=4 i b \eta_{(\mathbf{6}, \overline{\mathbf{6}})}^{\mathrm{PS}(\mathrm{S}), \mathbb{S}} \\
\delta^{\vec{a}} \eta_{(\mathbf{6}, \overline{\mathbf{6}})}^{\mathrm{S}(\mathrm{PS}), \mathbb{S}}=0
\end{gathered}
$$$$
\delta_{5}^{\vec{b}} \eta_{(\mathbf{6}, \overline{\mathbf{6}})}^{\mathrm{S}(\mathrm{PS}), \mathbb{S}}=4 i b^{N} \eta_{(\mathbf{6}, \overline{\mathbf{6}}), N}^{\mathrm{PS}(\mathrm{S}) \mathbb{Q}},
$$$$
\delta_{5} \eta_{(\mathbf{6}, \overline{\mathbf{6}}), N}^{\mathrm{S}(\mathrm{PS}, \mathbb{O}}=4 i b \eta_{(\mathbf{6}, \overline{\mathbf{6}}), N}^{\mathrm{PS}(\mathrm{S}) \mathbb{Q}}
$$

$$
\delta^{\vec{a}} \eta_{(\mathbf{6}, \overline{\mathbf{6}}), \mathrm{S}}^{\mathrm{S}(\mathrm{P}), \mathbb{Q}}=2 a^{N} f_{N M O} \eta_{(\mathbf{6}, \overline{\mathbf{6}}), \mathrm{O}}^{\mathrm{S}(\mathrm{PS}) \mathbb{O}}
$$

$$
\begin{aligned}
& \delta_{5}^{\vec{b}} \eta_{(\mathbf{6}, \overline{\mathbf{6}}), N}^{\mathrm{S}(\mathrm{PS}), \mathbb{Q}}=\frac{5}{3} i b^{M} \eta_{(\mathbf{6}, \overline{\mathbf{6}})}^{\mathrm{PS}(\mathrm{S}), S}+\frac{14}{5} i b^{N} d_{N M O} \eta_{(\mathbf{6}, \overline{\mathbf{6}}), \mathrm{O}}^{\mathrm{PS}(\mathrm{O}), \mathbb{O}} \\
& +4 i b^{N}\left(\mathbf{T}_{8 \times 27}\right)_{M U}^{N} \eta_{(\mathbf{6}, \overline{\mathbf{6}}), U}^{\mathrm{PS}(\mathrm{IS}}, \\
& \delta_{5} \eta_{(\mathbf{6}, \overline{\mathbf{6}})}^{\mathrm{S}(\mathrm{PS}, \mathbb{T S}}=4 i b \eta_{(\mathbf{6}, \overline{\mathbf{6}}), U}^{\mathrm{PS}, \mathrm{SS}},
\end{aligned}
$$

$$
\begin{aligned}
& \delta^{\vec{a}} \eta_{(\mathbf{6}, \overline{\mathbf{6}})}^{\mathrm{S}(\mathrm{PS}), \mathbb{S}}=2 i a^{N}\left(\mathbf{T}_{27 \times 27}^{\mathbf{A}}-\mathbf{T}_{27 \times 27}^{\mathbf{B}}\right)_{U V}^{N} \eta_{(\mathbf{6}, \overline{\mathbf{6}}), V}^{\mathrm{S}(\mathrm{PS}), \mathbb{S}}, \\
& \delta_{5}^{\vec{b}} \eta_{(\mathbf{6}, \overline{\mathbf{6}})}^{\mathrm{S}(\mathrm{PS}), \mathbb{I S}}=\frac{6}{5} i b^{N}\left(\mathbf{T}_{8 \times 27}^{\dagger}\right)_{U O}^{N} \eta_{(\mathbf{6}, \overline{\mathbf{6}}), \mathrm{O}}^{\mathrm{PS}(\mathrm{S}) \mathbb{O}} \\
& +2 i b^{N}\left(\mathbf{T}_{27 \times 27}^{\mathbf{A}}+\mathbf{T}_{27 \times 27}^{\mathbf{B}}\right)_{U V}^{N} \eta_{(\mathbf{6}, \overline{\mathbf{6}}), V}^{\mathrm{PS}(\mathrm{S}) \mathbb{S}} .
\end{aligned}
$$


There are two $[(\mathbf{8}, \mathbf{8}) \oplus(\mathbf{8}, \mathbf{8})]$ chiral multiplets, $\left(2 \eta_{7}^{\mathrm{S}, \mathrm{S}}+\right.$ $\eta_{10}^{\mathrm{S}, \mathrm{S}}, 2 \eta_{7}^{\mathrm{PS}, \mathrm{S}}+\eta_{10}^{\mathrm{PS}, \mathbb{S}}=0,5 \eta_{7, N}^{\mathrm{S}, \mathbb{O}}+\eta_{10, N}^{\mathrm{S}, \mathbb{O}}, \eta_{7, N}^{\mathrm{PS}, \mathbb{Q}}+\eta_{10, N}^{\mathrm{PS}, \mathbb{Q}}$, $\left.\eta_{5, U}^{\mathrm{S}, \mathrm{TS}}, \eta_{1, P}^{\mathrm{PS}, \mathbb{D}}, \eta_{2, P}^{\mathrm{PS}, \overline{\mathrm{D}}}\right)$, and $\left(2 \eta_{8}^{\mathrm{S}, \mathrm{S}}+\eta_{9}^{\mathrm{S}, \mathrm{S}}, 2 \eta_{8}^{\mathrm{PS}, \mathrm{S}}+\eta_{9}^{\mathrm{PS}, \mathrm{S}}=0\right.$, $\left.5 \eta_{8, N}^{\mathrm{S}, \mathbb{Q}}+\eta_{9, N}^{\mathrm{S}, \mathbb{Q}}, \eta_{8, N}^{\mathrm{PS}, \mathbb{Q}}+\eta_{9, N}^{\mathrm{PS}, \mathbb{Q}}, \eta_{4, U}^{\mathrm{S}, \mathbb{U S}}, \eta_{2, P}^{\mathrm{PS}, \mathbb{D}}, \eta_{1, P}^{\mathrm{PS}, \overline{\mathbb{D}}}\right)$. We use $\left(\eta_{(\mathbf{8}, \mathbf{8})}^{\mathrm{S}, \mathrm{S}}\right.$, $\left.\eta_{(\mathbf{8}, \mathbf{8})}^{\mathrm{PS}, \mathbb{S}}, \eta_{(\mathbf{8 , 8}), N}^{\mathrm{S}, \mathbb{O}}, \eta_{(\mathbf{8 , 8}), N}^{\mathrm{PS}, \mathbb{O}}, \eta_{(\mathbf{8 , 8}), U}^{\mathrm{S}, \mathbb{S S}}, \eta_{(\mathbf{8 , 8}), P}^{\mathrm{PS}, \mathbb{D}}, \eta_{(\mathbf{8 , 8}), P}^{\mathrm{PS}, \overline{\mathrm{D}}}\right)$ to denote them, and their chiral transformation properties are

$$
\begin{aligned}
& \delta_{5} \eta_{(\mathbf{8 , 8})}^{\mathrm{S}, \mathrm{S}}=0 \\
& \delta^{\vec{a}} \eta_{(\mathbf{8 , 8})}^{\mathrm{S}, \mathbb{S}}=0 \\
& \delta_{5}^{\vec{b}} \eta_{(\mathbf{8 , 8})}^{\mathrm{S}, S}=6 i b^{N} \eta_{(\mathbf{8}, \mathbf{8}), N}^{\mathrm{PS}, \mathbb{Q}} \\
& \delta_{5} \eta_{(8,8), N}^{\mathrm{S}, \mathbb{O}}=0, \\
& \delta^{\vec{a}} \eta_{(\mathbf{8 , 8}), N}^{\mathrm{S}, \mathbb{O}}=2 a^{N} f_{N M O} \eta_{(\mathbf{8 , 8}), O}^{\mathrm{S}, \mathbb{O}} \\
& \delta_{5}^{\vec{b}} \eta_{(\mathbf{8}, \mathbf{8}), N}^{\mathrm{S}, \mathbb{Q}}=6 i b^{N} d_{N M O} \eta_{(\mathbf{8 , 8}), \mathrm{O}}^{\mathrm{PS}, \mathbb{Q}}-12 i b^{N}\left(\mathbf{T}_{8 \times 10}\right)_{M P}^{N} \eta_{(\mathbf{8 , 8}), P}^{\mathrm{PS}, \mathbb{D}} \\
& -12 i b^{N}\left(\mathbf{T}_{8 \times 10}^{*}\right)_{M P}^{N} \eta_{(\mathbf{8}, 8), P}^{\mathrm{PS}, \overline{\mathrm{D}}}, \\
& \delta_{5} \eta_{(\mathbf{8}, \mathbf{8}), N}^{\mathrm{PS}, \mathbb{Q}}=0 \\
& \delta^{\vec{a}} \eta_{(\mathbf{8 , 8}, \mathbf{8}, N}^{\mathrm{PS}, \mathbb{O}}=2 a^{N} f_{N M O} \eta_{(\mathbf{8 , 8}), O}^{\mathrm{PS}, \mathbb{O}} \\
& \delta_{5}^{\vec{b}} \eta_{(\mathbf{8 , 8}), N}^{\mathrm{PS}, \mathbb{O}}=i b^{M} \eta_{(\mathbf{8 , 8})}^{\mathrm{S}, \mathbb{S}}+\frac{6}{5} i b^{N} d_{N M O} \eta_{(\mathbf{8}, \mathbf{8}), \mathrm{O}}^{\mathrm{S}, \mathbb{O}} \\
& -4 i b^{N}\left(\mathbf{T}_{8 \times 27}\right)_{M U}^{N} \eta_{(\mathbf{8 , 8}), U}^{\mathrm{S}, \mathbb{S}}, \\
& \delta_{5} \eta_{(\mathbf{8 , 8}), U}^{\mathrm{S}, \mathbb{S}}=0 \\
& \delta^{\vec{a}} \eta_{(\mathbf{8}, \mathbf{8}), U}^{\mathrm{S}, \mathbb{S}}=2 i a^{N}\left(\mathbf{T}_{27 \times 27}^{\mathbf{A}}-\mathbf{T}_{27 \times 27}^{\mathbf{B}}\right)_{U V}^{N} \eta_{(\mathbf{8}, \mathbf{8}), V}^{\mathrm{S}, \mathbb{S}} \\
& \delta_{5}^{\vec{b}} \eta_{(\mathbf{8}, \mathbf{8}), U}^{\mathrm{S}, \mathbb{S}}=-i b^{N}\left(\mathbf{T}_{8 \times 27}^{\dagger}\right)_{U O}^{N} \eta_{(\mathbf{8 , 8}), \mathrm{O}}^{\mathrm{PS}, \mathbb{Q}} \\
& +3 i b^{N}\left(\mathbf{T}_{10 \times 27}^{\mathbf{B} \dagger}\right)_{U P}^{N} \eta_{(\mathbf{8}, \mathbf{8}), P}^{\mathrm{PS}, \overline{\mathrm{D}}} \\
& +3 i b^{N}\left(\mathbf{T}_{10 \times 27}^{\mathbf{A} \dagger}\right)_{U P}^{N} \eta_{(\mathbf{8}, \mathbf{8}), P}^{\mathrm{PS}, \mathbb{D}}
\end{aligned}
$$

$$
\begin{aligned}
& \delta_{5} \eta_{(\mathbf{8}, \mathbf{8}), P}^{\mathrm{PS}, \mathbb{D}}=0 \\
& \delta^{\vec{a}} \eta_{(\mathbf{8 , 8}), P}^{\mathrm{PS}, \mathbb{D}}=3 i a^{N}\left(\mathbf{T}_{10 \times 10}\right)_{P Q}^{N} \eta_{(\mathbf{8 , 8}), Q^{\prime}}^{\mathrm{PS}, \mathbb{D}} \\
& \delta_{5}^{\vec{b}} \eta_{(\mathbf{8}, \mathbf{8}), P}^{\mathrm{PS}, \mathbb{D}}=-\frac{2}{5} i b^{N}\left(\mathbf{T}_{8 \times 10}^{\dagger}\right)_{P O}^{N} \eta_{(\mathbf{8}, \mathbf{8}), \mathrm{O}}^{\mathrm{S}, \mathbb{O}} \\
& +2 i b^{N}\left(\mathbf{T}_{10 \times 27}^{\mathbf{A}}\right)_{P U}^{N} \eta_{(\mathbf{8 , 8}), U}^{\mathrm{S}, \mathbb{S}}, \\
& \delta_{5} \eta_{(\mathbf{8 , 8}), P}^{\mathrm{PS}, \overline{\mathbb{D}}}=0, \\
& \delta^{\vec{a}} \eta_{(\mathbf{8}, \mathbf{8}), P}^{\mathrm{PS}, \overline{\mathrm{D}}}=-3 i a^{N}\left(\mathbf{T}_{10 \times 10}^{*}\right)_{P Q}^{N} \eta_{(\mathbf{8}, \mathbf{8}), \mathrm{Q}}^{\mathrm{PS}, \overline{\mathrm{D}}}, \\
& \delta_{5}^{\vec{b}} \eta_{(\mathbf{8}, \mathbf{8}), P}^{\mathrm{PS}, \overline{\mathrm{D}}}=-\frac{2}{5} i b^{N}\left(\mathbf{T}_{8 \times 10}^{\dagger *}\right)_{P O}^{N} \eta_{(\mathbf{8 , 8}), \mathrm{O}}^{\mathrm{S}, \mathrm{O}} \\
& +2 i b^{N}\left(\mathbf{T}_{10 \times 27}^{\mathbf{B}}\right)_{P U}^{N} \eta_{(\mathbf{8 , 8}), U}^{\mathrm{S}, \mathbb{S}} .
\end{aligned}
$$

There are four $[(\mathbf{8}, \mathbf{1}) \oplus(\mathbf{1}, \mathbf{8})]$ chiral multiplets, $\left(\eta_{1, N}^{\mathrm{S}, \mathbb{O}}-\eta_{2, N}^{\mathrm{S}, \mathbb{O}}\right.$, $\left.\eta_{1, N}^{\mathrm{PS}, \mathbb{O}}-\eta_{2, N}^{\mathrm{PS}, \mathbb{O}}\right),\left(\eta_{4, N}^{\mathrm{S}, \mathbb{O}}-\eta_{5, N}^{\mathrm{S}, \mathbb{O}}, \eta_{4, N}^{\mathrm{PS}, \mathbb{O}}-\eta_{5, N}^{\mathrm{PS}, \mathbb{O}}\right),\left(\eta_{7, N}^{\mathrm{S}, \mathbb{O}}-\eta_{10, N}^{\mathrm{S}, \mathbb{O}}, \eta_{7, N}^{\mathrm{PS}, \mathbb{O}}-\right.$ $\left.\eta_{10, N}^{\text {PS, }}\right),\left(\eta_{8, N}^{\text {S, }}-\eta_{9, N}^{\text {S, }}, \eta_{8, N}^{\text {PS, }}-\eta_{9, N}^{\text {PS,O }}\right)$. We use $\left(\eta_{(8,1), N}^{\text {S, }}, \eta_{(8,1), N}^{\text {PS, }}\right)$ to denote them, and their chiral transformation properties are

$$
\begin{gathered}
\delta_{5} \eta_{(\mathbf{8}, \mathbf{1}), N}^{\mathrm{S}(\mathrm{PS}), \mathbb{Q}}=0, \\
\delta^{\vec{a}} \eta_{(\mathbf{8}, \mathbf{1}), N}^{\mathrm{S}(\mathrm{PS}), \mathbb{Q}}=2 a^{N} f_{N M O} \eta_{(\mathbf{8}, \mathbf{1}), \mathrm{O}}^{\mathrm{S}(\mathrm{PS}) \mathbb{Q}}, \\
\delta_{5}^{\vec{b}} \eta_{(\mathbf{8}, \mathbf{1}), N}^{\mathrm{S}(\mathrm{PS}), \mathbb{Q}}=2 b^{N} f_{N M O} \eta_{(\mathbf{8}, \mathbf{1}), \mathrm{O}}^{\mathrm{PS}(\mathrm{O}),}
\end{gathered}
$$

There is only one $[(27, \mathbf{1}) \oplus(\mathbf{1}, \mathbf{2 7})]$ chiral multiplet, $\left(\eta_{1, U}^{\mathrm{S}, \mathbb{U S}}-\right.$ $\left.\eta_{2, U}^{\mathrm{S}, \mathbb{\mathbb { S }}}, \eta_{1, U}^{\mathrm{PS}, \mathbb{S S}}-\eta_{2, U}^{\mathrm{PS}, \mathbb{T S}}\right)$. Its chiral transformation properties are

$$
\begin{gathered}
\delta_{5} \eta_{1-2, U}^{\mathrm{S}(\mathrm{PS}), \mathbb{T S}}=0, \\
\delta^{\vec{a}} \eta_{1-2, U}^{\mathrm{S}(\mathrm{PS}) \mathbb{U} S}=2 i a^{N}\left(\mathbf{T}_{27 \times 27}^{\mathbf{A}}-\mathbf{T}_{27 \times 27}^{\mathbf{B}}\right)_{U V}^{N} \eta_{1-2, V}^{\mathrm{S}(\mathrm{PS}) \mathbb{U S}}, \\
\delta_{5}^{\vec{b}} \eta_{1-2, U}^{\mathrm{S}(\mathrm{PS}), \mathbb{U S}}=2 i a^{N}\left(\mathbf{T}_{27 \times 27}^{\mathbf{A}}-\mathbf{T}_{27 \times 27}^{\mathbf{B}}\right)_{U V}^{N} \eta_{1-2, V}^{\mathrm{PS}(\mathrm{S}), \mathbb{S} S} .
\end{gathered}
$$




\section{Transition Matrices}

The transition matrices $\mathbf{T}_{10 \times 10}^{N}$ are

$$
\begin{aligned}
& \mathbf{T}_{10 \times 10}^{1}=\left(\begin{array}{cccccccccc}
0 & \frac{1}{\sqrt{3}} & 0 & 0 & 0 & 0 & 0 & 0 & 0 & 0 \\
\frac{1}{\sqrt{3}} & 0 & \frac{2}{3} & 0 & 0 & 0 & 0 & 0 & 0 & 0 \\
0 & \frac{2}{3} & 0 & \frac{1}{\sqrt{3}} & 0 & 0 & 0 & 0 & 0 & 0 \\
0 & 0 & \frac{1}{\sqrt{3}} & 0 & 0 & 0 & 0 & 0 & 0 & 0 \\
0 & 0 & 0 & 0 & 0 & \frac{\sqrt{2}}{3} & 0 & 0 & 0 & 0 \\
0 & 0 & 0 & 0 & \frac{\sqrt{2}}{3} & 0 & \frac{\sqrt{2}}{3} & 0 & 0 & 0 \\
0 & 0 & 0 & 0 & 0 & \frac{\sqrt{2}}{3} & 0 & 0 & 0 & 0 \\
0 & 0 & 0 & 0 & 0 & 0 & 0 & 0 & \frac{1}{3} & 0 \\
0 & 0 & 0 & 0 & 0 & 0 & 0 & \frac{1}{3} & 0 & 0 \\
0 & 0 & 0 & 0 & 0 & 0 & 0 & 0 & 0 & 0
\end{array}\right), \\
& \mathbf{T}_{10 \times 10}^{2}=\left(\begin{array}{cccccccccc}
0 & -\frac{i}{\sqrt{3}} & 0 & 0 & 0 & 0 & 0 & 0 & 0 & 0 \\
\frac{i}{\sqrt{3}} & 0 & -\frac{2 i}{3} & 0 & 0 & 0 & 0 & 0 & 0 & 0 \\
0 & \frac{2 i}{3} & 0 & -\frac{i}{\sqrt{3}} & 0 & 0 & 0 & 0 & 0 & 0 \\
0 & 0 & \frac{i}{\sqrt{3}} & 0 & 0 & 0 & 0 & 0 & 0 & 0 \\
0 & 0 & 0 & 0 & 0 & -\frac{i \sqrt{2}}{3} & 0 & 0 & 0 & 0 \\
0 & 0 & 0 & 0 & \frac{i \sqrt{2}}{3} & 0 & -\frac{i \sqrt{2}}{3} & 0 & 0 & 0 \\
0 & 0 & 0 & 0 & 0 & \frac{i \sqrt{2}}{3} & 0 & 0 & 0 & 0 \\
0 & 0 & 0 & 0 & 0 & 0 & 0 & 0 & -\frac{i}{3} & 0 \\
0 & 0 & 0 & 0 & 0 & 0 & 0 & \frac{i}{3} & 0 & 0 \\
0 & 0 & 0 & 0 & 0 & 0 & 0 & 0 & 0 & 0
\end{array}\right), \\
& \mathbf{T}_{10 \times 10}^{3}=\left(\begin{array}{cccccccccc}
1 & 0 & 0 & 0 & 0 & 0 & 0 & 0 & 0 & 0 \\
0 & \frac{1}{3} & 0 & 0 & 0 & 0 & 0 & 0 & 0 & 0 \\
0 & 0 & -\frac{1}{3} & 0 & 0 & 0 & 0 & 0 & 0 & 0 \\
0 & 0 & 0 & -1 & 0 & 0 & 0 & 0 & 0 & 0 \\
0 & 0 & 0 & 0 & \frac{2}{3} & 0 & 0 & 0 & 0 & 0 \\
0 & 0 & 0 & 0 & 0 & 0 & 0 & 0 & 0 & 0 \\
0 & 0 & 0 & 0 & 0 & 0 & -\frac{2}{3} & 0 & 0 & 0 \\
0 & 0 & 0 & 0 & 0 & 0 & 0 & \frac{1}{3} & 0 & 0 \\
0 & 0 & 0 & 0 & 0 & 0 & 0 & 0 & -\frac{1}{3} & 0 \\
0 & 0 & 0 & 0 & 0 & 0 & 0 & 0 & 0 & 0
\end{array}\right),
\end{aligned}
$$




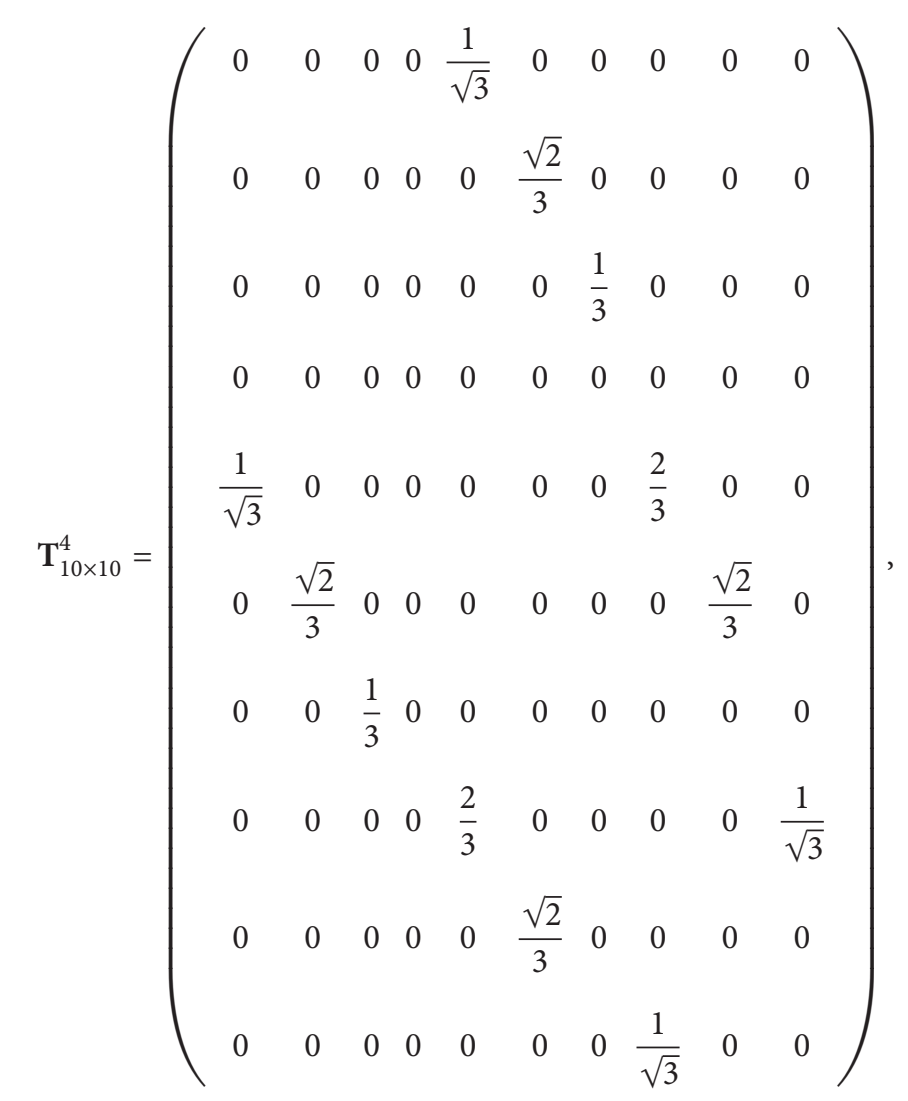

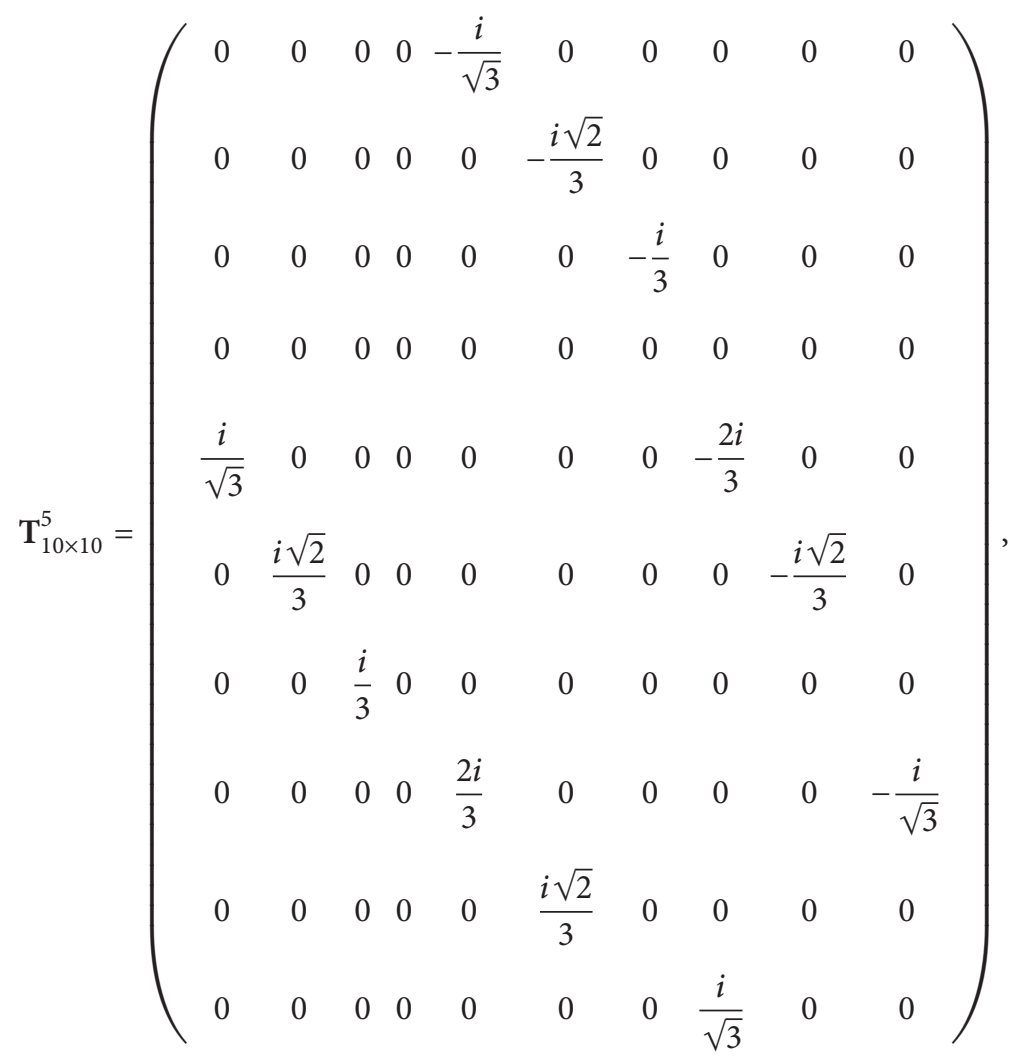




$$
\begin{aligned}
& \mathbf{T}_{10 \times 10}^{6}=\left(\begin{array}{cccccccccc}
0 & 0 & 0 & 0 & 0 & 0 & 0 & 0 & 0 & 0 \\
0 & 0 & 0 & 0 & \frac{1}{3} & 0 & 0 & 0 & 0 & 0 \\
0 & 0 & 0 & 0 & 0 & \frac{\sqrt{2}}{3} & 0 & 0 & 0 & 0 \\
0 & 0 & 0 & 0 & 0 & 0 & \frac{1}{\sqrt{3}} & 0 & 0 & 0 \\
0 & \frac{1}{3} & 0 & 0 & 0 & 0 & 0 & 0 & 0 & 0 \\
0 & 0 & 0 & \frac{1}{\sqrt{3}} & 0 & 0 & 0 & 0 & \frac{2}{3} & 0 \\
0 & 0 & 0 & 0 & 0 & \frac{\sqrt{2}}{3} & 0 & 0 & 0 & 0 \\
0 & 0 & 0 & 0 & 0 & 0 & \frac{2}{3} & 0 & 0 & \frac{1}{\sqrt{3}} \\
0 & 0 & 0 & 0 & 0 & 0 & 0 & 0 & \frac{1}{\sqrt{3}} & 0
\end{array}\right), \\
& \mathbf{T}_{10 \times 10}^{7}=\left(\begin{array}{cccccccccc}
0 & 0 & 0 & 0 & 0 & 0 & 0 & 0 & 0 & 0 \\
0 & 0 & 0 & 0 & -\frac{i}{3} & 0 & 0 & 0 & 0 & 0 \\
0 & 0 & 0 & 0 & 0 & -\frac{i \sqrt{2}}{3} & 0 & 0 & 0 & 0 \\
0 & 0 & 0 & 0 & 0 & 0 & -\frac{i}{\sqrt{3}} & 0 & 0 & 0 \\
0 & \frac{i}{3} & 0 & 0 & 0 & 0 & 0 & 0 & 0 & 0 \\
0 & 0 & \frac{i \sqrt{2}}{3} & 0 & 0 & 0 & 0 & -\frac{i \sqrt{2}}{3} & 0 & 0 \\
0 & 0 & 0 & \frac{i}{\sqrt{3}} & 0 & 0 & 0 & 0 & -\frac{2 i}{3} & 0 \\
0 & 0 & 0 & 0 & 0 & \frac{i \sqrt{2}}{3} & 0 & 0 & 0 & 0 \\
0 & 0 & 0 & 0 & 0 & 0 & \frac{2 i}{3} & 0 & 0 & -\frac{i}{\sqrt{3}} \\
0 & 0 & 0 & 0 & 0 & 0 & 0 & 0 & \frac{i}{\sqrt{3}} & 0
\end{array}\right), \\
& \mathbf{T}_{10 \times 10}^{8}=\left(\begin{array}{cccccccccc}
\frac{1}{\sqrt{3}} & 0 & 0 & 0 & 0 & 0 & 0 & 0 & 0 & 0 \\
0 & \frac{1}{\sqrt{3}} & 0 & 0 & 0 & 0 & 0 & 0 & 0 & 0 \\
0 & 0 & \frac{1}{\sqrt{3}} & 0 & 0 & 0 & 0 & 0 & 0 & 0 \\
0 & 0 & 0 & \frac{1}{\sqrt{3}} & 0 & 0 & 0 & 0 & 0 & 0 \\
0 & 0 & 0 & 0 & 0 & 0 & 0 & 0 & 0 & 0 \\
0 & 0 & 0 & 0 & 0 & 0 & 0 & 0 & 0 & 0 \\
0 & 0 & 0 & 0 & 0 & 0 & 0 & 0 & 0 & 0 \\
0 & 0 & 0 & 0 & 0 & 0 & 0 & -\frac{1}{\sqrt{3}} & 0 & 0 \\
0 & 0 & 0 & 0 & 0 & 0 & 0 & 0 & -\frac{1}{\sqrt{3}} & 0 \\
0 & 0 & 0 & 0 & 0 & 0 & 0 & 0 & 0 & -\frac{2}{\sqrt{3}}
\end{array}\right) .
\end{aligned}
$$


The transition matrices $\left(\mathbf{T}_{10 \times 27}^{A}\right)^{N \dagger}$ are

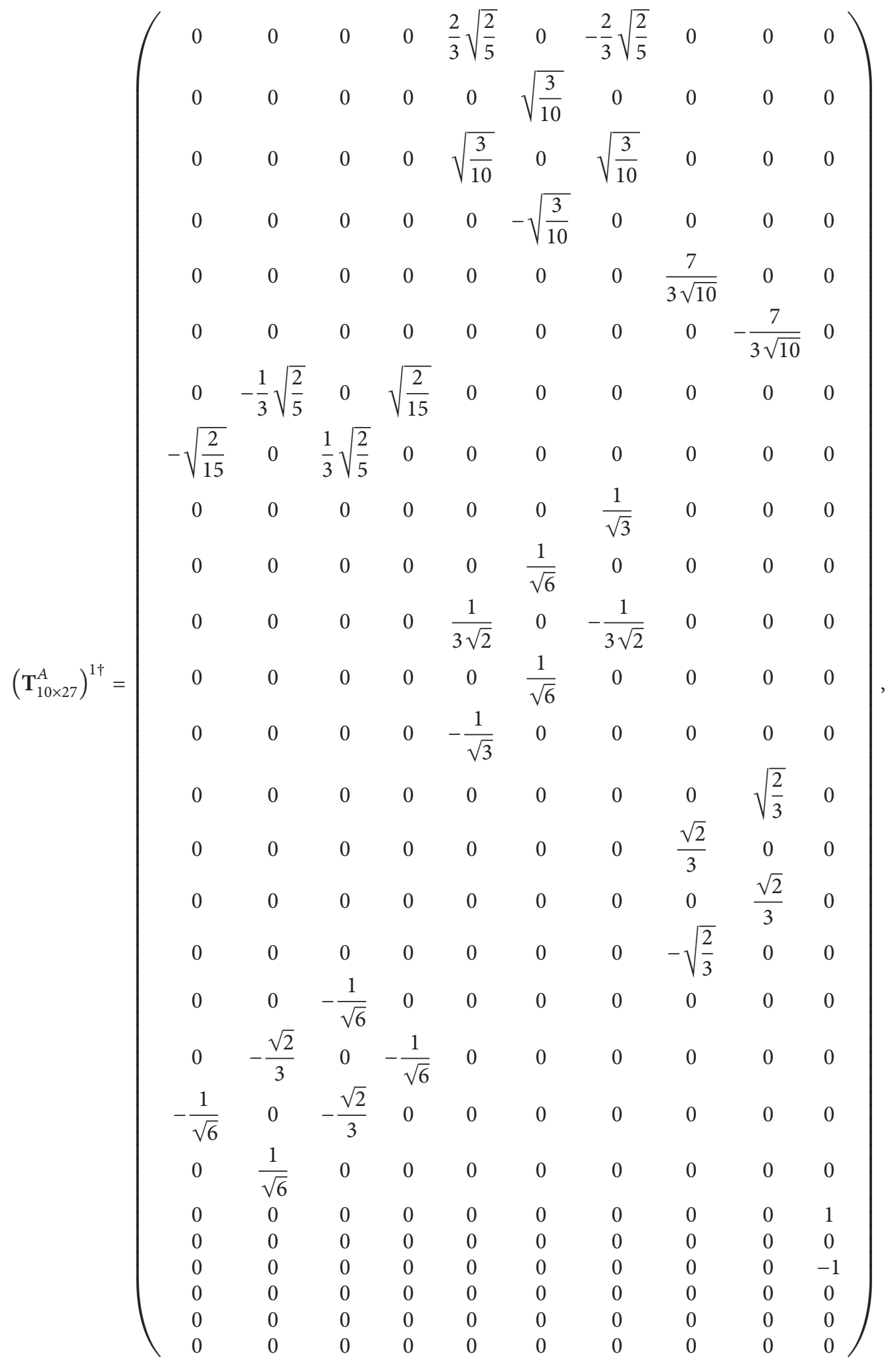




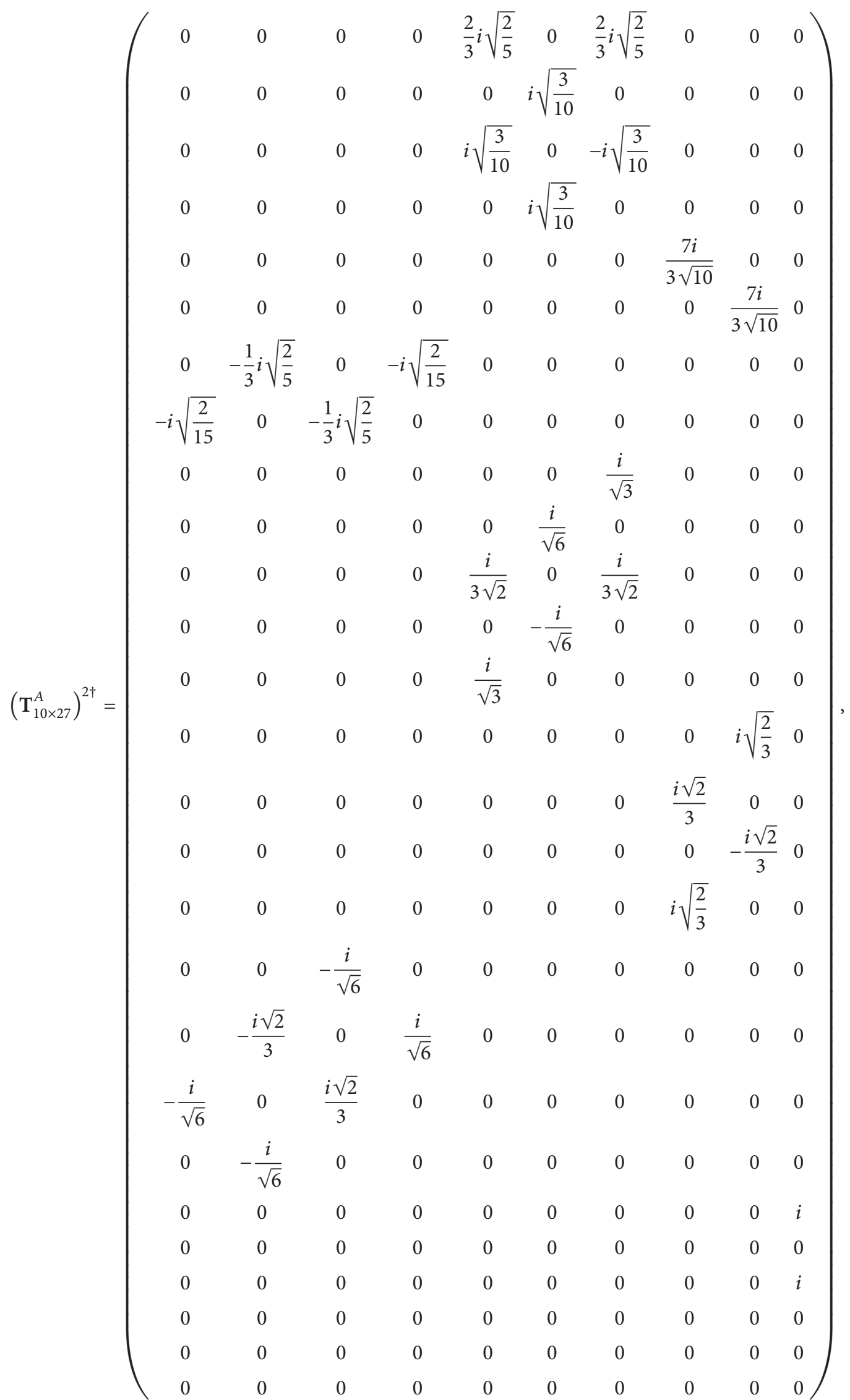




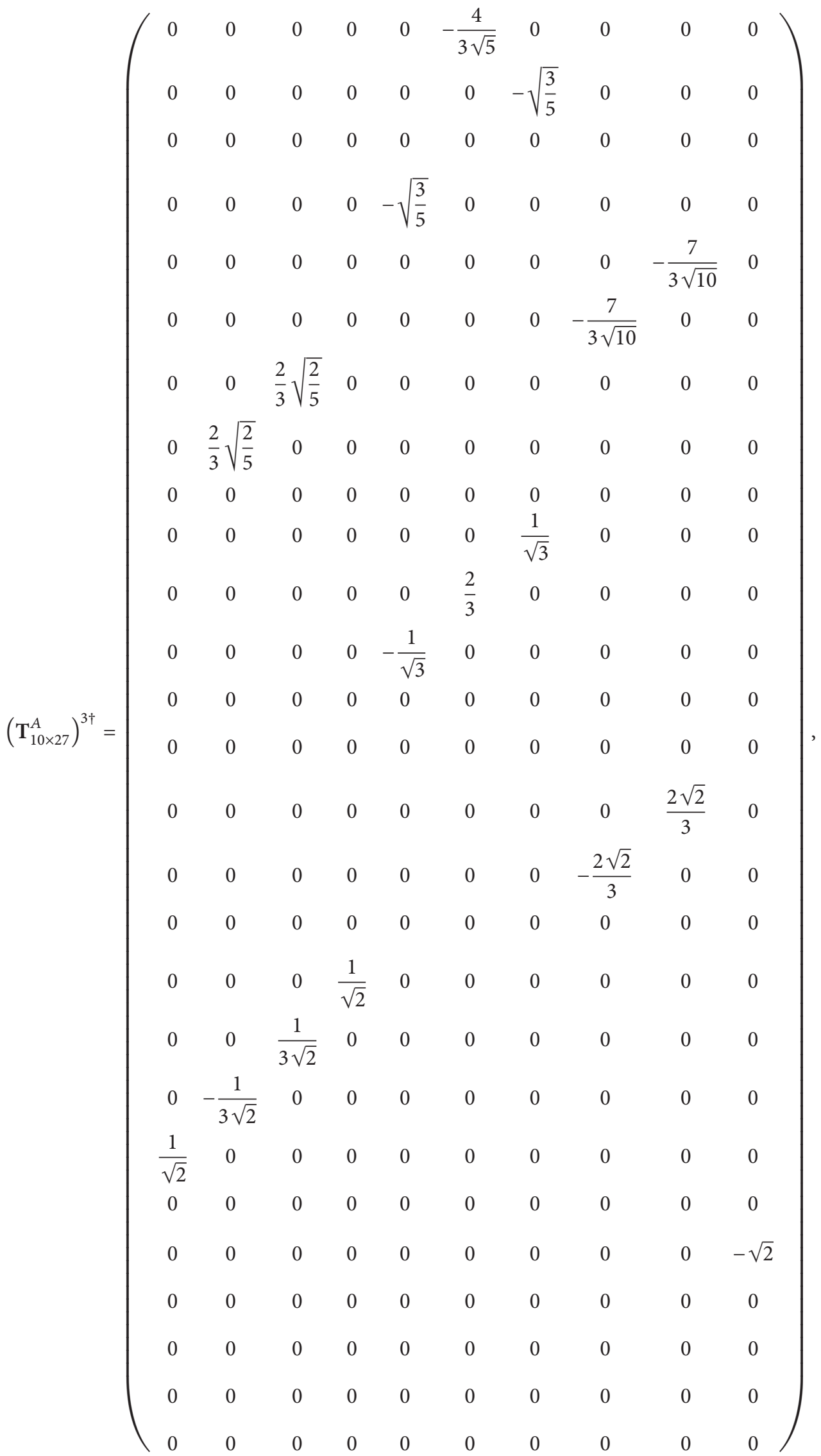




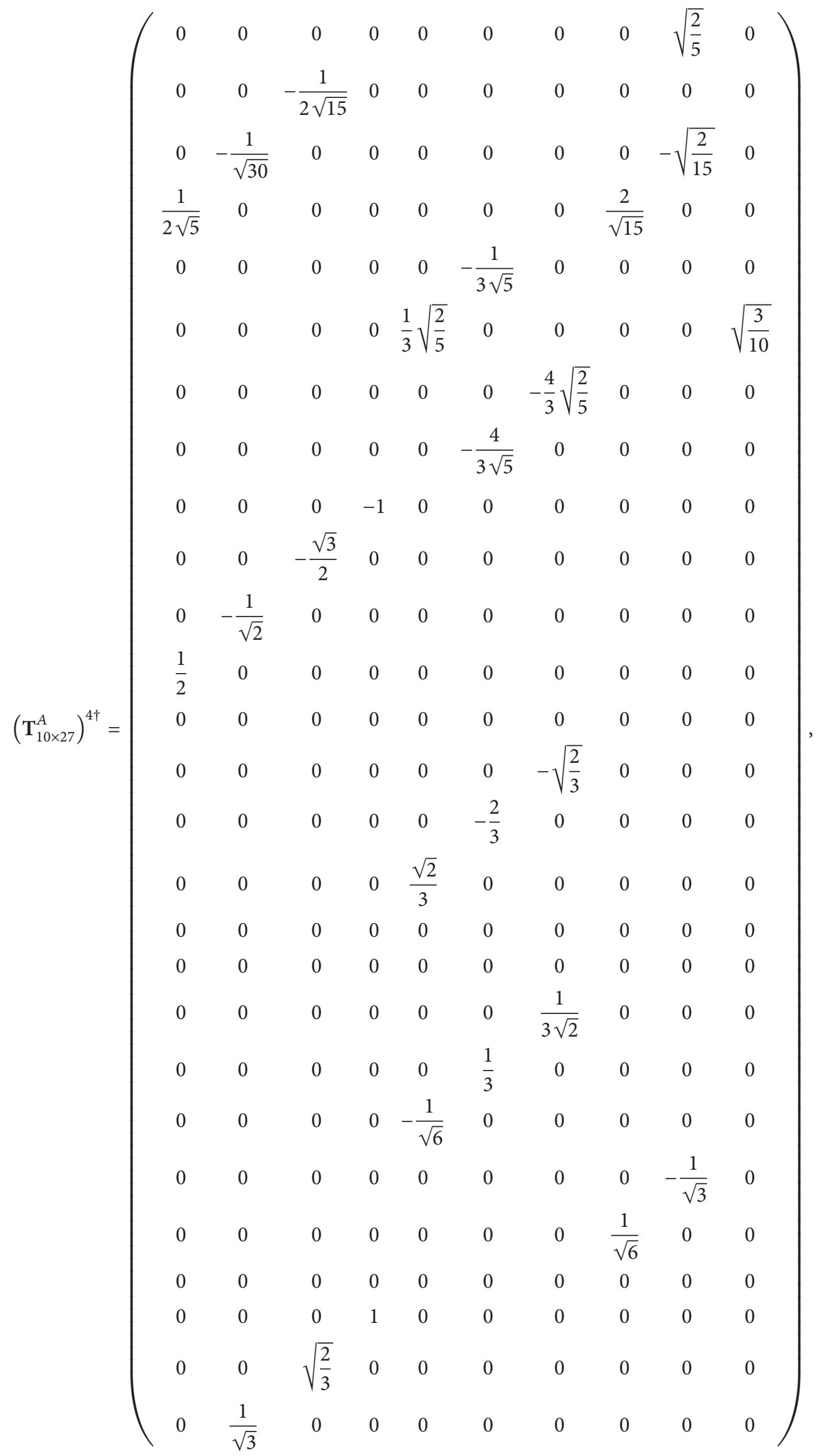




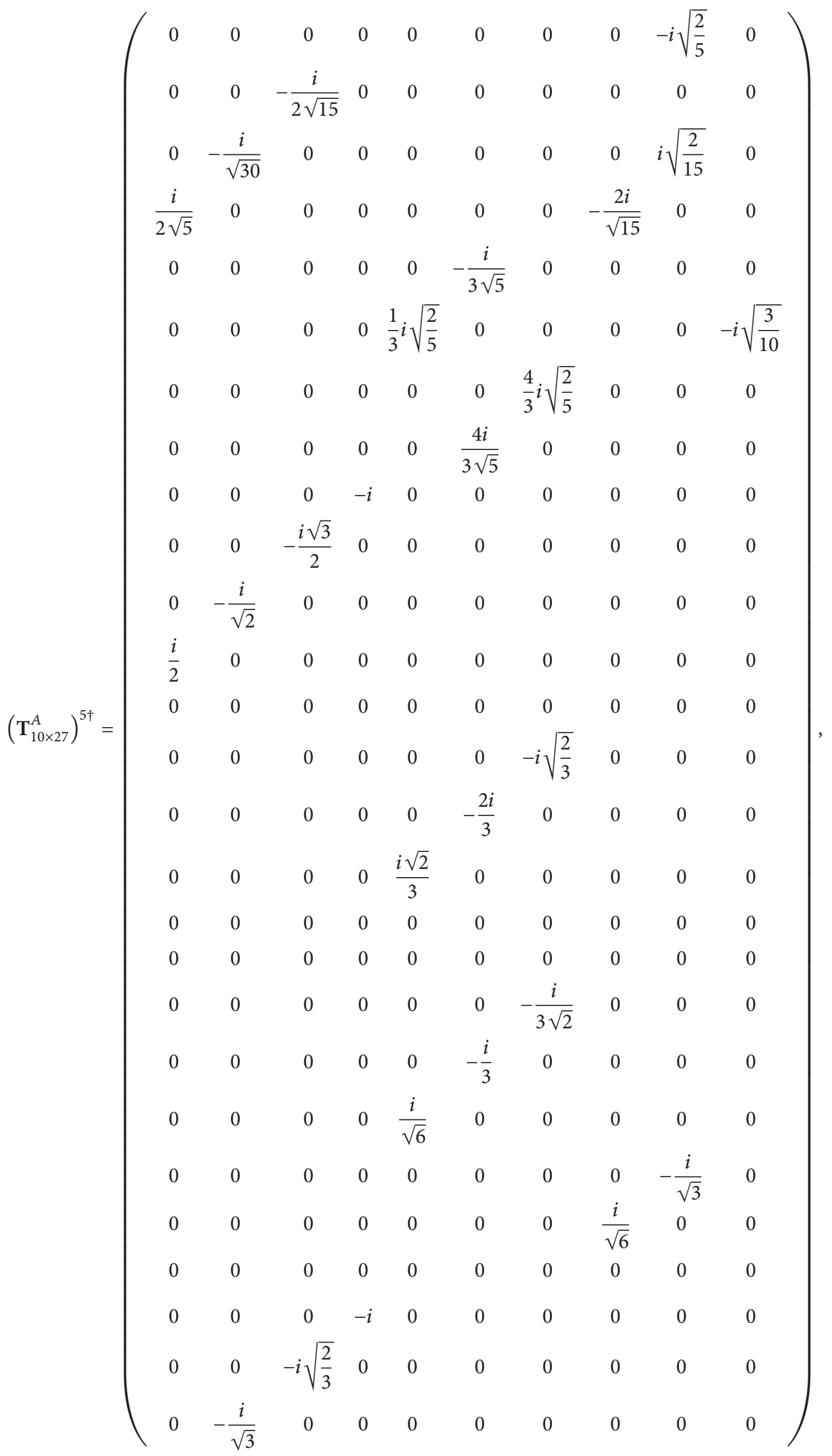




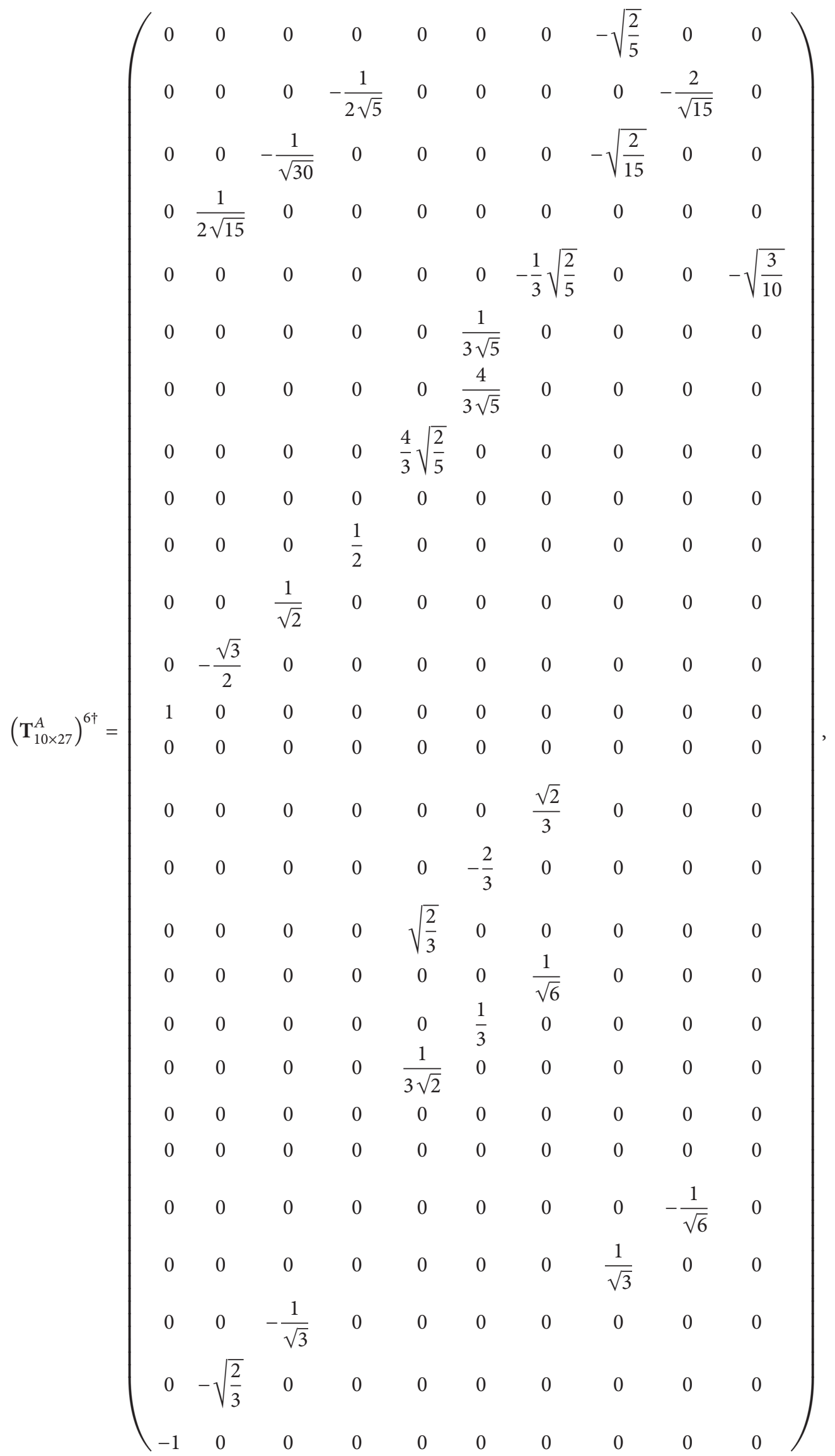




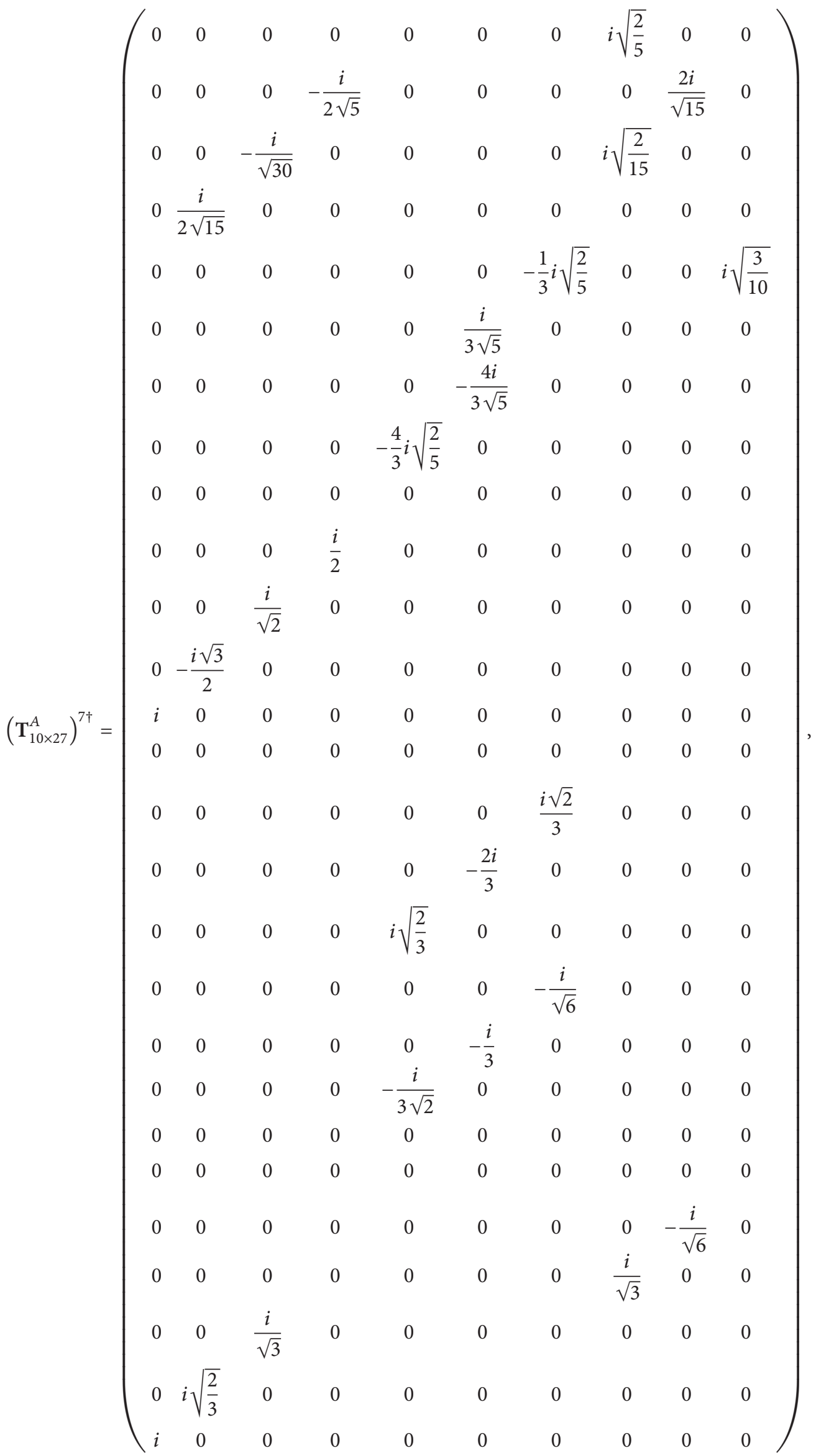




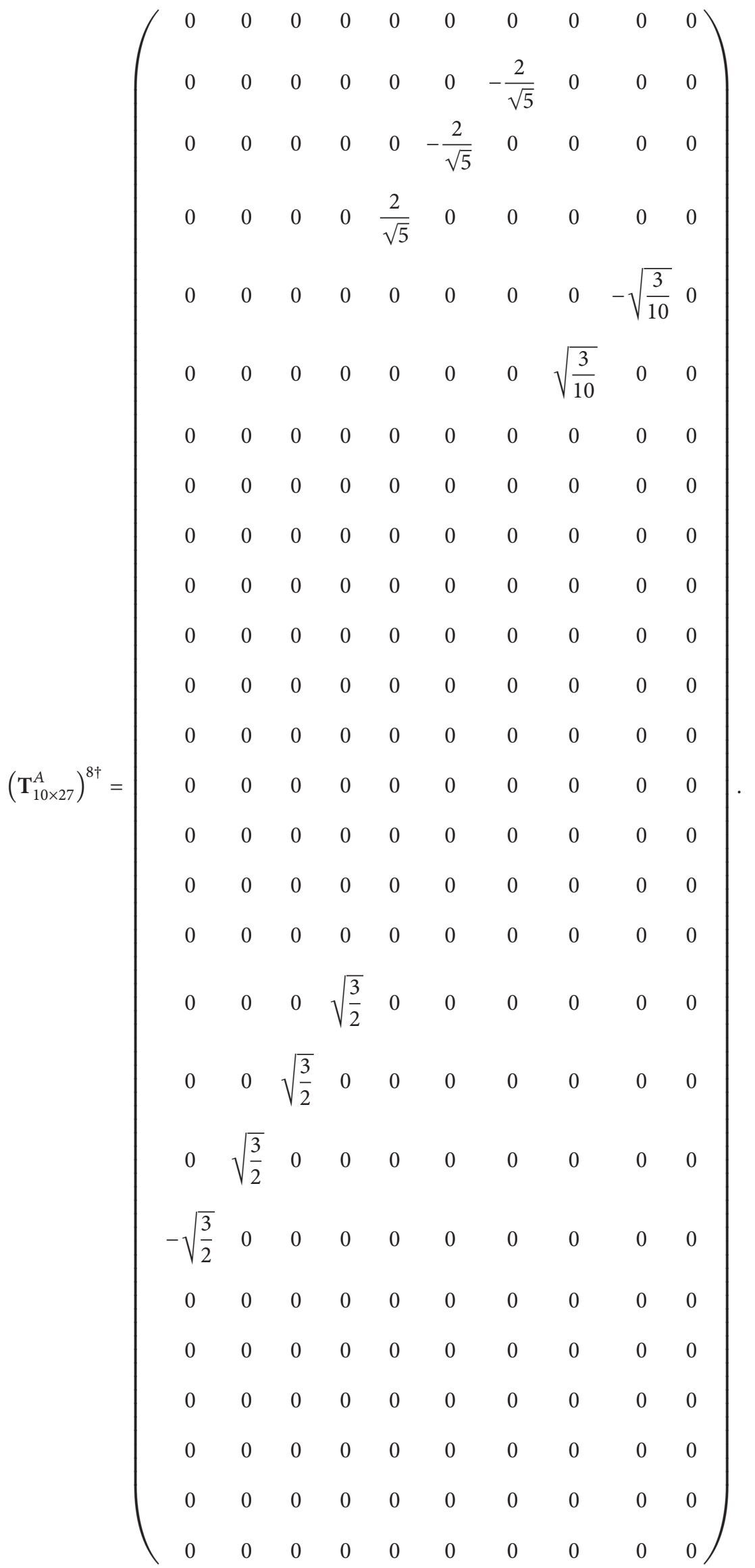


The transition matrices $\left(\mathbf{T}_{10 \times 27}^{B}\right)^{N \dagger}$ are

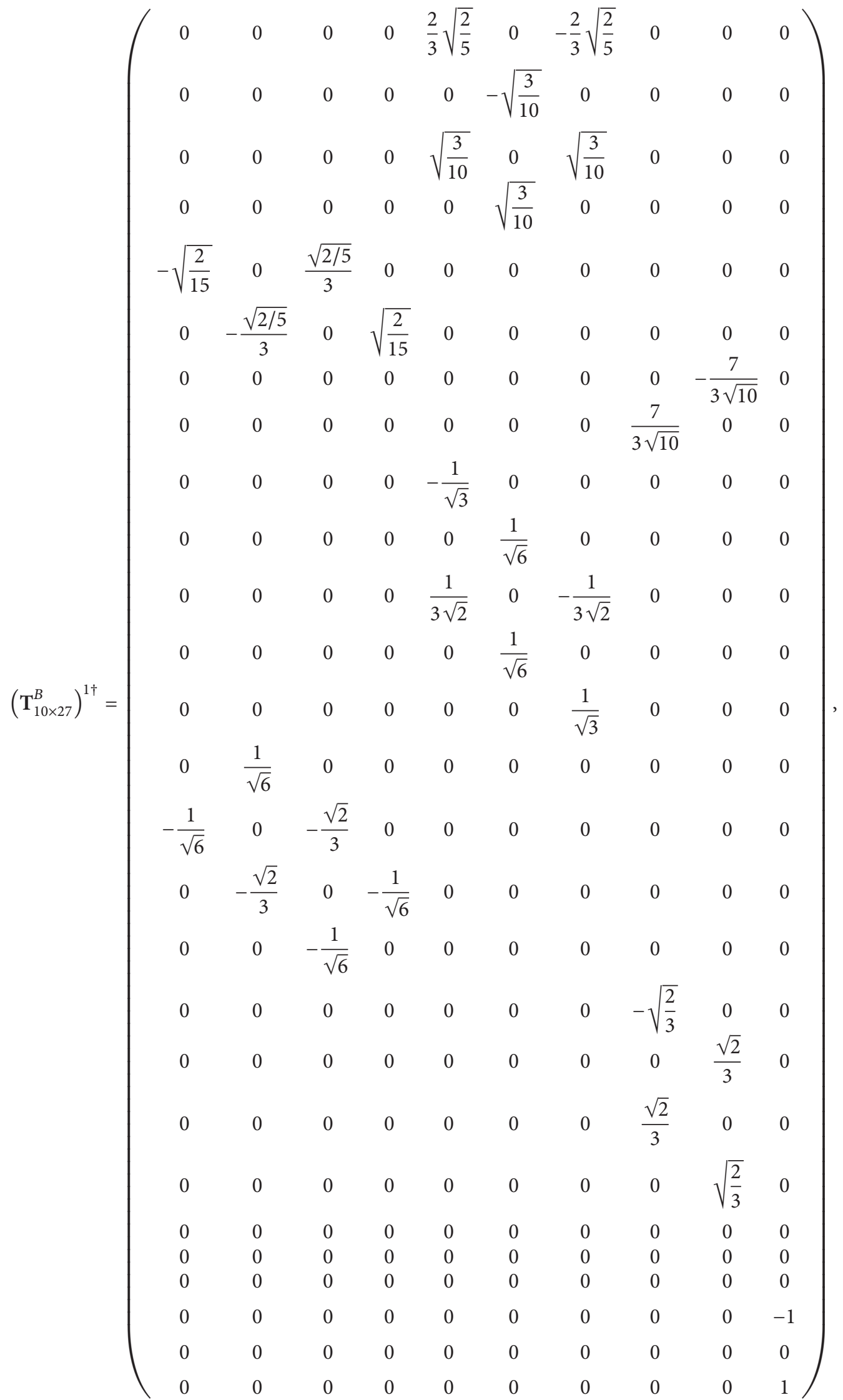




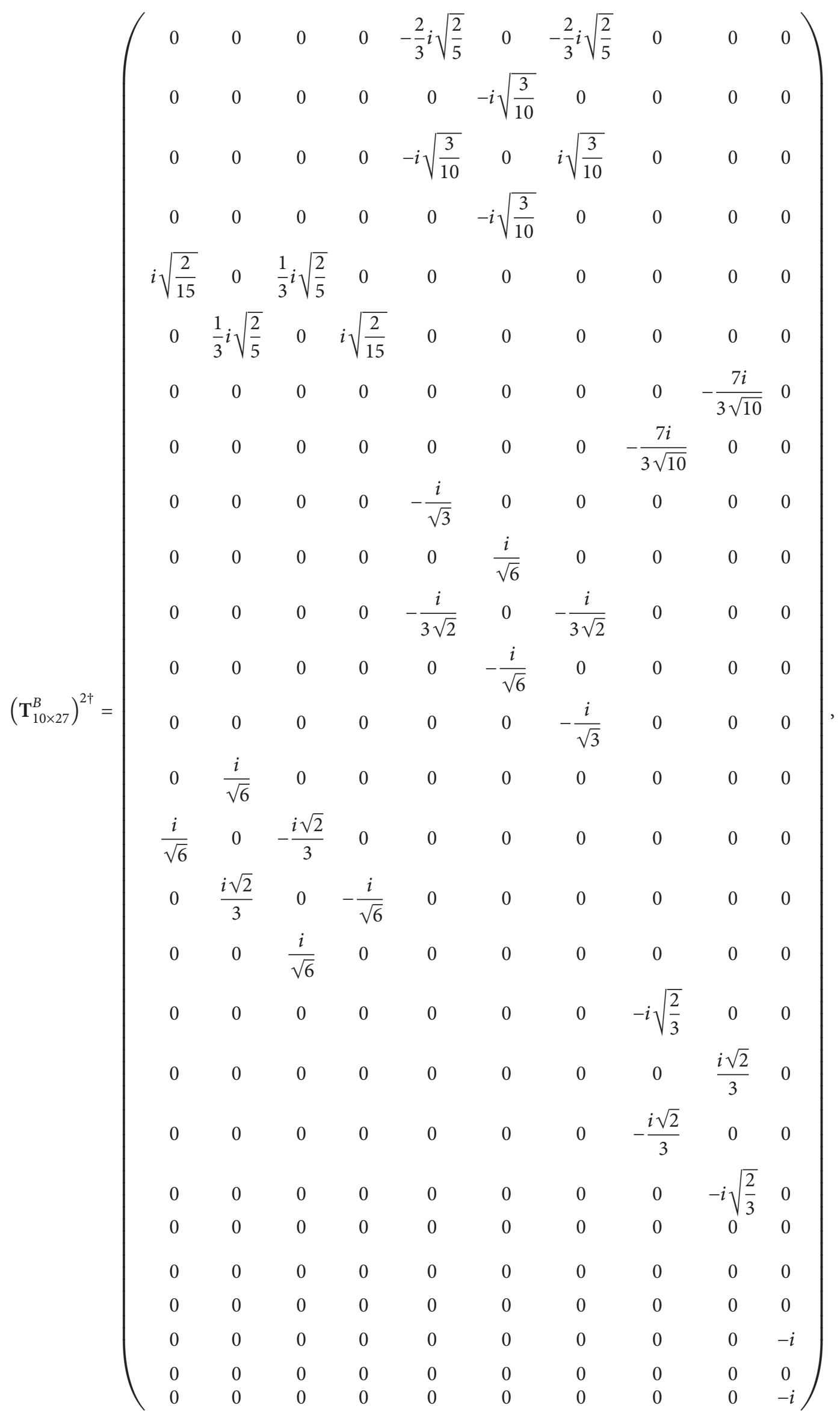




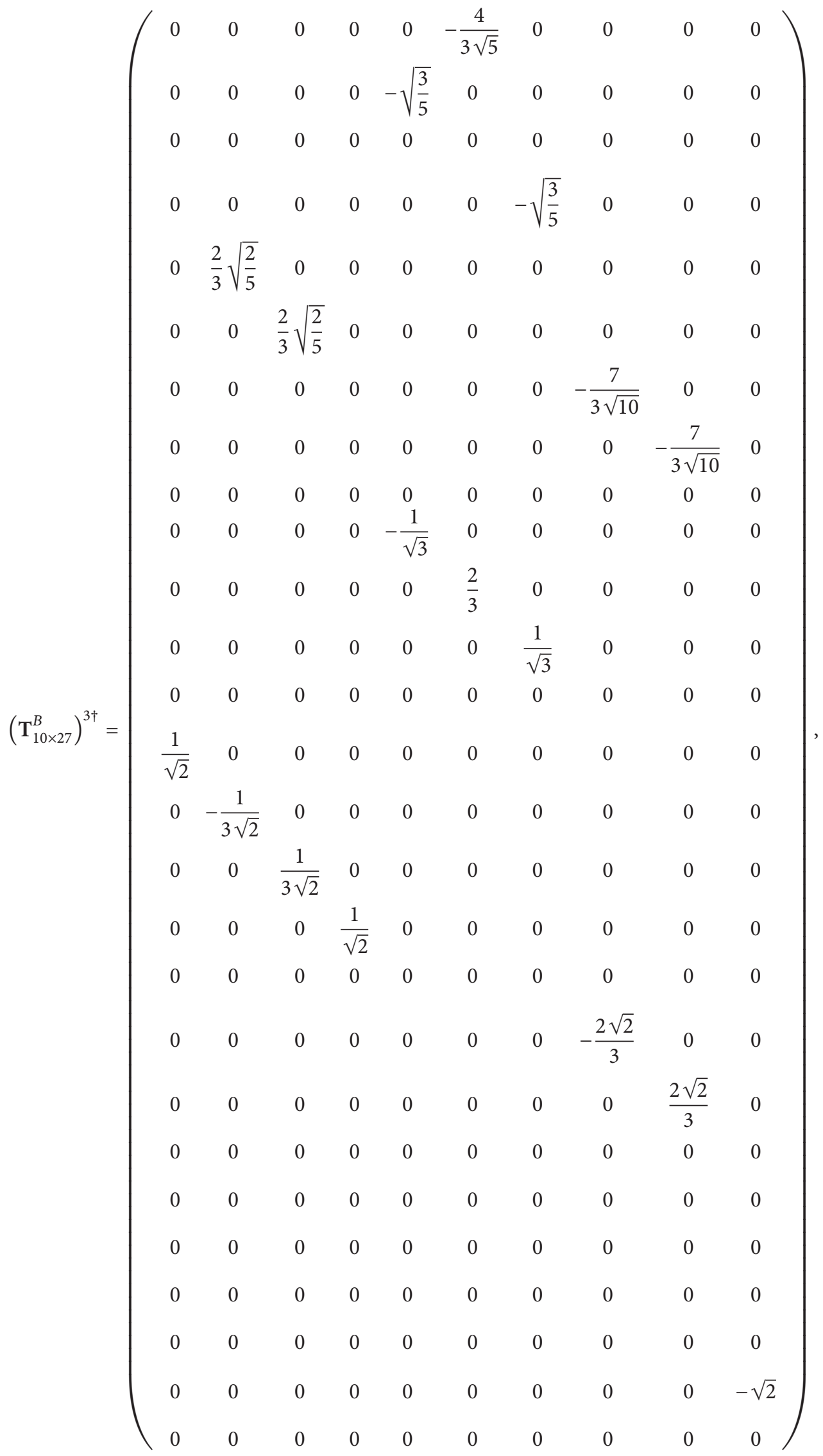




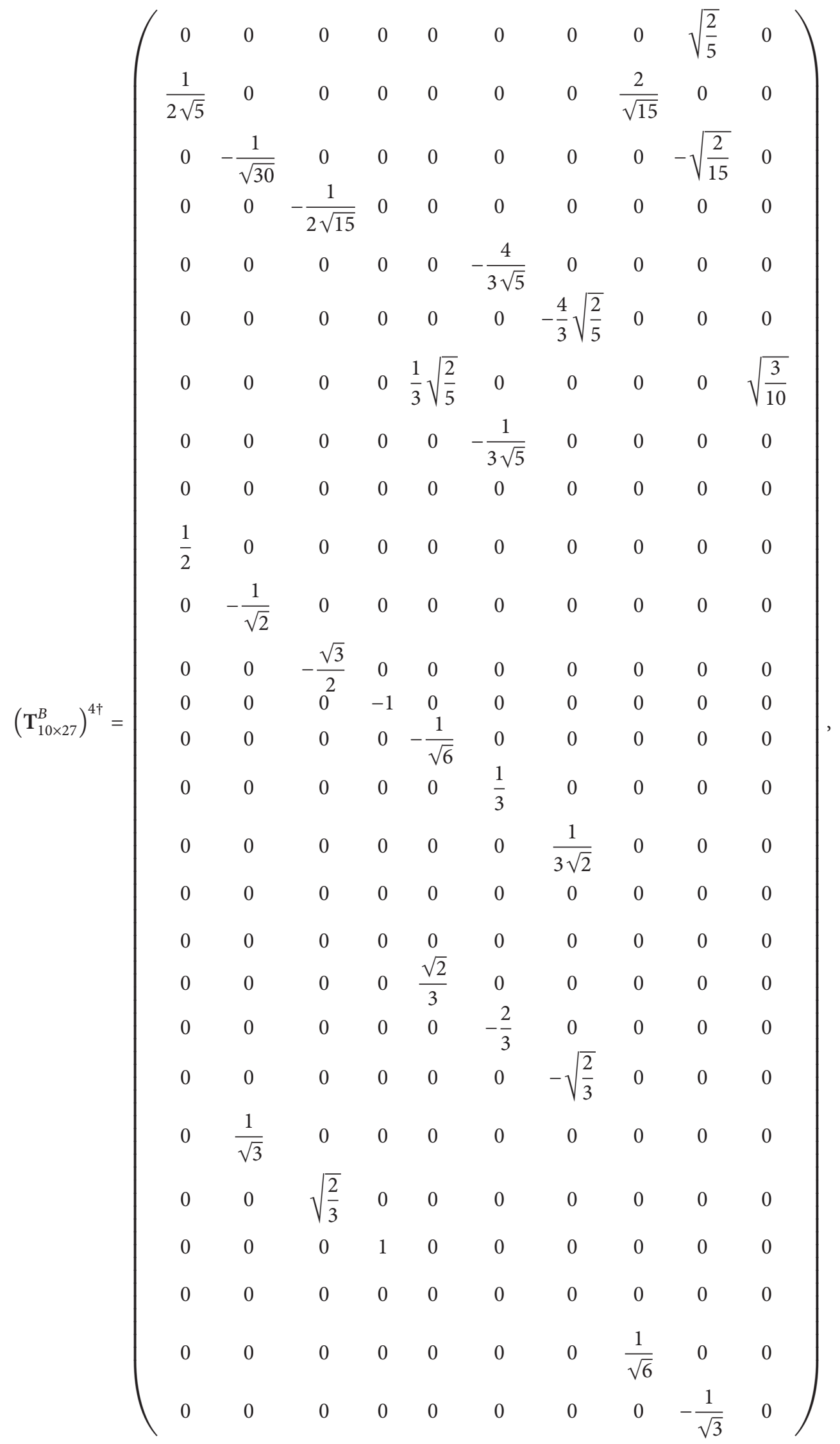




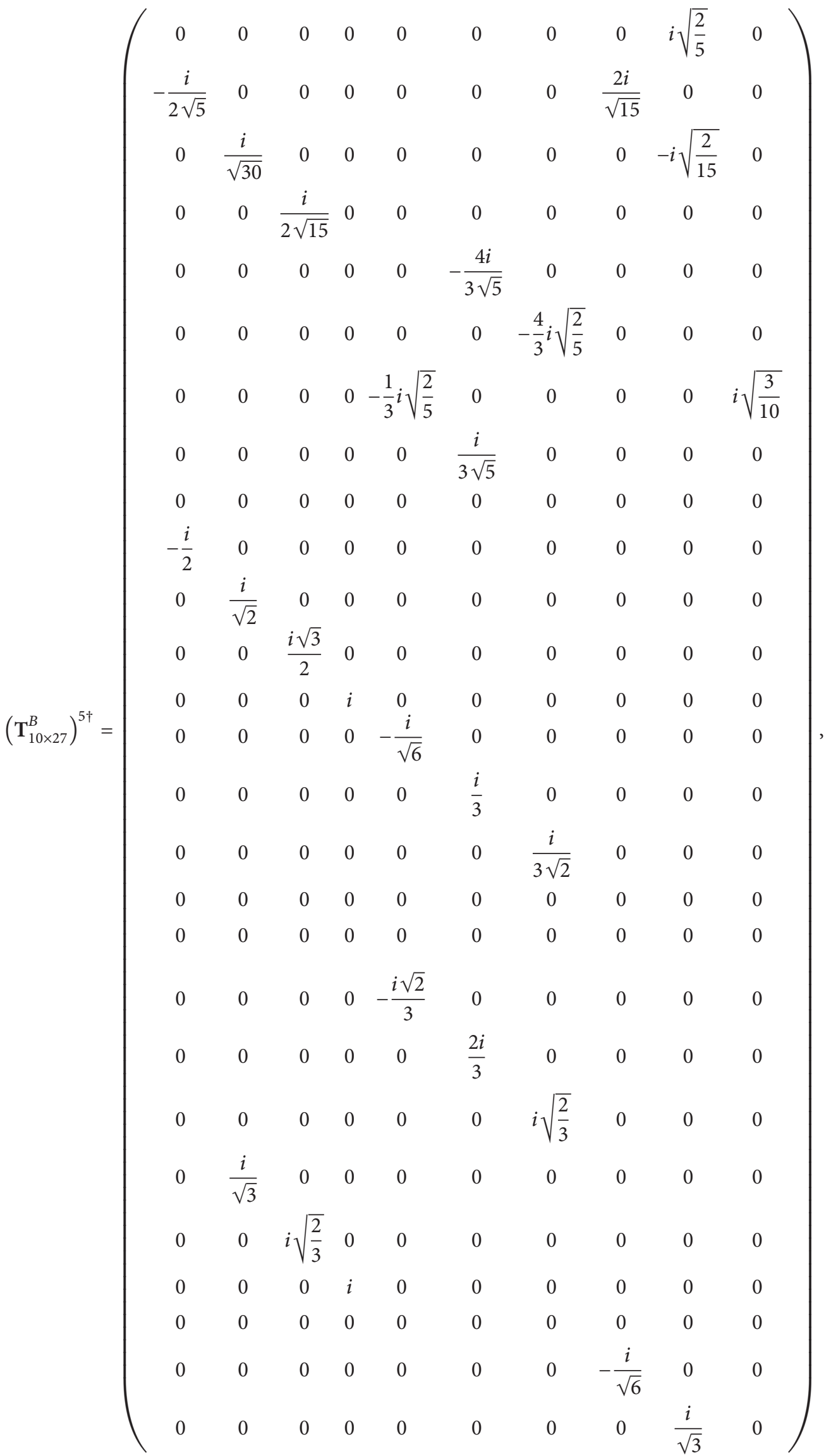




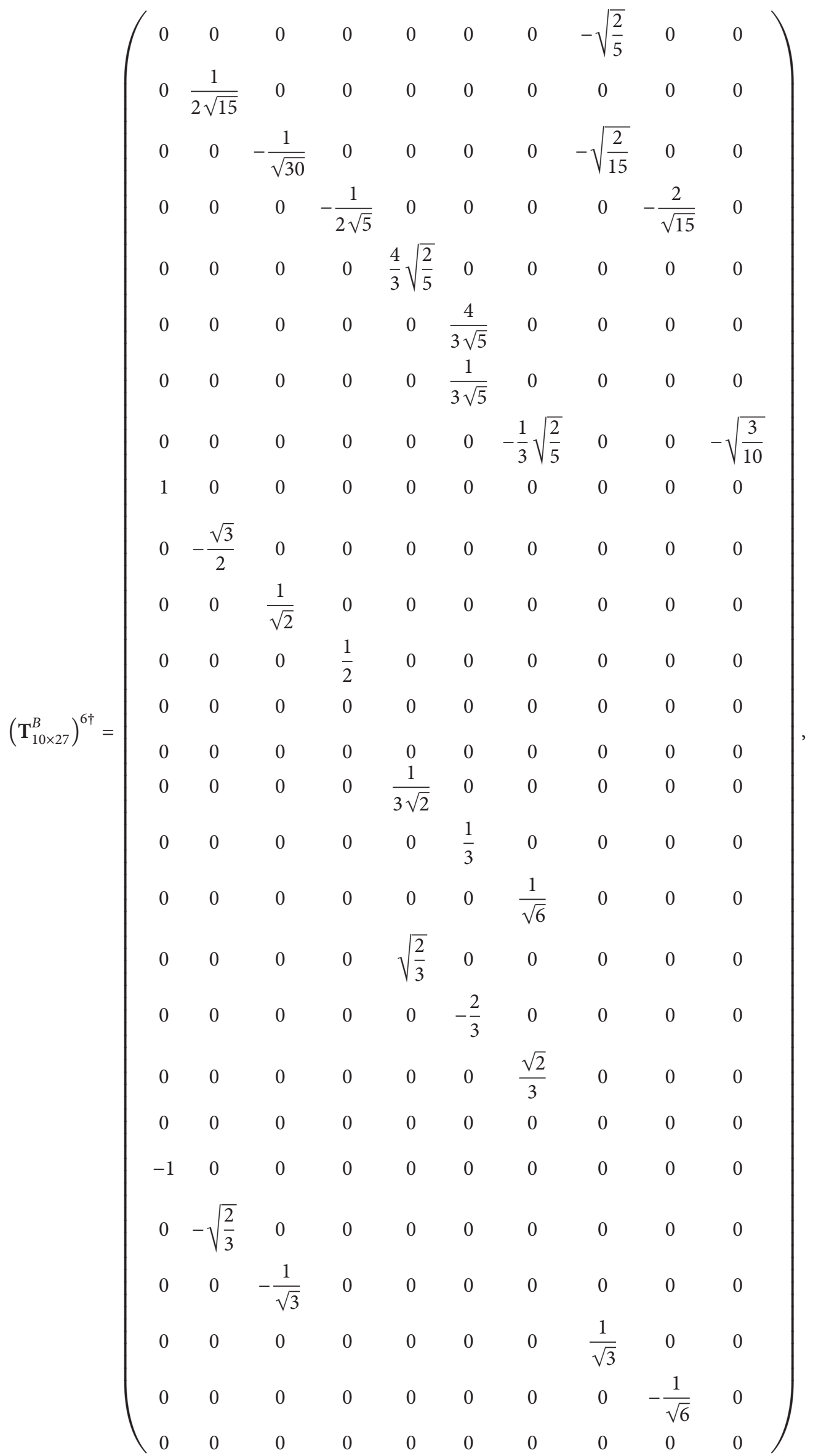




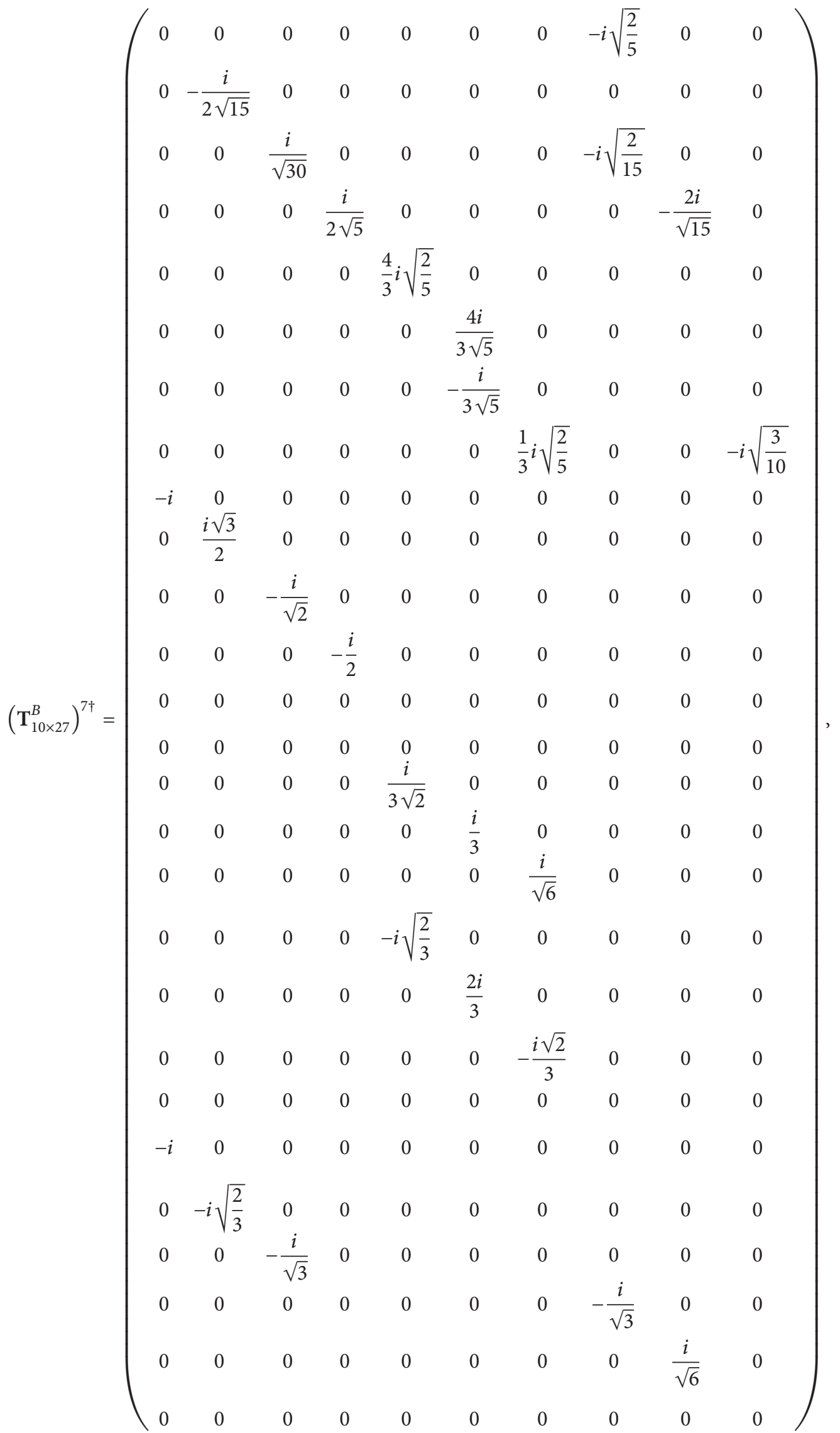




$$
\left(\mathbf{T}_{10 \times 27}^{B}\right)^{8 \dagger}=\left(\begin{array}{cccccccccc}
0 & 0 & 0 & 0 & 0 & 0 & 0 & 0 & 0 & 0 \\
0 & 0 & 0 & 0 & \frac{2}{\sqrt{5}} & 0 & 0 & 0 & 0 & 0 \\
0 & 0 & 0 & 0 & 0 & -\frac{2}{\sqrt{5}} & 0 & 0 & 0 & 0 \\
0 & 0 & 0 & 0 & 0 & 0 & -\frac{2}{\sqrt{5}} & 0 & 0 & 0 \\
0 & 0 & 0 & 0 & 0 & 0 & 0 & 0 & 0 & 0 \\
0 & 0 & 0 & 0 & 0 & 0 & 0 & 0 & 0 & 0 \\
0 & 0 & 0 & 0 & 0 & 0 & 0 & \sqrt{\frac{3}{10}} & 0 & 0 \\
0 & 0 & 0 & 0 & 0 & 0 & 0 & 0 & -\sqrt{\frac{3}{10}} & 0 \\
0 & 0 & 0 & 0 & 0 & 0 & 0 & 0 & 0 & 0 \\
0 & 0 & 0 & 0 & 0 & 0 & 0 & 0 & 0 & 0 \\
0 & 0 & 0 & 0 & 0 & 0 & 0 & 0 & 0 & 0 \\
0 & 0 & 0 & 0 & 0 & 0 & 0 & 0 & 0 & 0 \\
-\sqrt{\frac{3}{2}} & 0 & 0 & 0 & 0 & 0 & 0 & 0 & 0 & 0 \\
0 & \sqrt{\frac{3}{2}} & 0 & 0 & 0 & 0 & 0 & 0 & 0 & 0 \\
0 & 0 & \sqrt{\frac{3}{2}} & 0 & 0 & 0 & 0 & 0 & 0 & 0 \\
0 & 0 & 0 & \sqrt{\frac{3}{2}} & 0 & 0 & 0 & 0 & 0 & 0 \\
0 & 0 & 0 & 0 & 0 & 0 & 0 & 0 & 0 & 0 \\
0 & 0 & 0 & 0 & 0 & 0 & 0 & 0 & 0 & 0 \\
0 & 0 & 0 & 0 & 0 & 0 & 0 & 0 & 0 & 0 \\
0 & 0 & 0 & 0 & 0 & 0 & 0 & 0 & 0 & 0 \\
0 & 0 & 0 & 0 & 0 & 0 & 0 & 0 & 0 & 0 \\
0 & 0 & 0 & 0 & 0 & 0 & 0 & 0 & 0 & 0 \\
0 & 0 & 0 & 0 & 0 & 0 & 0 & 0 & 0 & 0 \\
0 & 0 & 0 & 0 & 0 & 0 & 0 & 0 & 0 & 0 \\
0 & 0 & 0 & 0 & 0 & 0 & 0 & 0 & 0 & 0 \\
0 & 0 & 0 & 0 & 0 & 0 & 0 & 0 & 0 & 0
\end{array}\right) .
$$

\section{D. $(\bar{q} q)(\bar{q} q)$ Tetraquark Currents}

In Section 2 and Appendix A we have investigated the chiral structure of local scalar and pseudoscalar tetraquark currents constructed using diquarks and antidiquarks, while they can also be constructed using two quark-antiquark pairs. These two different constructions can be related to each other through Fierz transformations, and so they can equally describe the full space of local tetraquark currents. In this appendix we show these relations. We note that some of these relations have been obtained in $[42,60,69]$.

We shall separately investigate scalar and pseudoscalar in the following subsections. Since Fierz transformations can only change the Lorentz structure and not change the flavor symmetry and the color symmetry of diquarks and antidiquarks, we shall fix these two symmetries in the following discussions, and separately study tetraquark currents having the antisymmetric flavor structure $\overline{\mathbf{3}}_{f}(q q) \otimes \mathbf{3}_{f}(\bar{q} \bar{q})$, the symmetric flavor structure $\mathbf{6}_{f}(q q) \otimes \overline{\mathbf{6}}_{f}(\bar{q} \bar{q})$, and the mixed flavor structure $\overline{\mathbf{3}}_{f}(q q) \otimes \overline{\mathbf{6}}_{f}(\bar{q} \bar{q})$. The other case of mixed flavor structure $\mathbf{6}_{f}(q q) \otimes \mathbf{3}_{f}(\bar{q} \bar{q})$ can be similarly investigated.

\section{D.1. Scalar Tetraquark Currents}

D.1.1. Flavor Structure $\overline{\mathbf{3}}_{f}(q q) \otimes \mathbf{3}_{f}(\bar{q} \bar{q})$. In this subsection we study the scalar tetraquark currents which contain diquarks and antidiquarks having both the antisymmetric flavor structure $\overline{\mathbf{3}} \otimes \mathbf{3}$. There are altogether five independent scalar tetraquark currents constructed using diquarks and antidiquarks:

$$
\begin{gathered}
\eta_{1}=q_{A}^{a T} \mathbb{C} \gamma_{5} q_{B}^{b}\left(\bar{q}_{C}^{a} \gamma_{5} \mathbb{C} \bar{q}_{D}^{b T}-\bar{q}_{C}^{b} \gamma_{5} \mathbb{C} \bar{q}_{D}^{a T}\right), \\
\eta_{2}=q_{A}^{a T} \mathbb{C} \gamma_{\mu} \gamma_{5} q_{B}^{b}\left(\bar{q}_{C}^{a} \gamma^{\mu} \gamma_{5} \mathbb{C} \bar{q}_{D}^{b T}-\bar{q}_{C}^{b} \gamma^{\mu} \gamma_{5} \mathbb{C} \bar{q}_{D}^{a T}\right), \\
\eta_{3}=q_{A}^{a T} \mathbb{C} \sigma_{\mu \nu} q_{B}^{b}\left(\bar{q}_{C}^{a} \sigma^{\mu \nu} \mathbb{C} \bar{q}_{D}^{b T}+\bar{q}_{C}^{b} \sigma^{\mu \nu} \mathbb{C} \bar{q}_{D}^{a T}\right),
\end{gathered}
$$




$$
\begin{gathered}
\eta_{4}=q_{A}^{a T} \mathbb{C} \gamma_{\mu} q_{B}^{b}\left(\bar{q}_{C}^{a} \gamma^{\mu} \mathbb{C} \bar{q}_{D}^{b T}+\bar{q}_{C}^{b} \gamma^{\mu} \mathbb{C} \bar{q}_{D}^{a T}\right), \\
\eta_{5}=q_{A}^{a T} \mathbb{C} q_{B}^{b}\left(\bar{q}_{C}^{a} \mathbb{C} \bar{q}_{D}^{b T}-\bar{q}_{C}^{b} \mathbb{C} \bar{q}_{D}^{a T}\right) .
\end{gathered}
$$

There are altogether ten scalar tetraquark currents constructed using quark-antiquark pairs:

$$
\begin{gathered}
\psi_{1}=\left(\bar{q}_{C}^{a} q_{A}^{a}\right)\left(\bar{q}_{D}^{b} q_{B}^{b}\right)-\left(\bar{q}_{C}^{a} q_{B}^{a}\right)\left(\bar{q}_{D}^{b} q_{A}^{b}\right), \\
\psi_{2}=\left(\bar{q}_{C}^{a} \gamma_{\mu} q_{A}^{a}\right)\left(\bar{q}_{D}^{b} \gamma^{\mu} q_{B}^{b}\right)-\left(\bar{q}_{C}^{a} \gamma_{\mu} q_{B}^{a}\right)\left(\bar{q}_{D}^{b} \gamma^{\mu} q_{A}^{b}\right), \\
\psi_{3}=\left(\bar{q}_{C}^{a} \sigma_{\mu \nu} q_{A}^{a}\right)\left(\bar{q}_{D}^{b} \sigma^{\mu \nu} q_{B}^{b}\right)-\left(\bar{q}_{C}^{a} \sigma_{\mu \nu} q_{B}^{a}\right)\left(\bar{q}_{D}^{b} \sigma^{\mu \nu} q_{A}^{b}\right), \\
\psi_{4}=\left(\bar{q}_{C}^{a} \gamma_{\mu} \gamma_{5} q_{A}^{a}\right)\left(\bar{q}_{D}^{b} \gamma^{\mu} \gamma_{5} q_{B}^{b}\right)-\left(\bar{q}_{C}^{a} \gamma_{\mu} \gamma_{5} q_{B}^{a}\right)\left(\bar{q}_{D}^{b} \gamma^{\mu} \gamma_{5} q_{A}^{b}\right), \\
\psi_{5}=\left(\bar{q}_{C}^{a} \gamma_{5} q_{A}^{a}\right)\left(\bar{q}_{D}^{b} \gamma_{5} q_{B}^{b}\right)-\left(\bar{q}_{C}^{a} \gamma_{5} q_{B}^{a}\right)\left(\bar{q}_{D}^{b} \gamma_{5} q_{A}^{b}\right), \\
\psi_{6}=\left(\bar{q}_{C}^{a} \lambda_{a b} q_{A}^{b}\right)\left(\bar{q}_{D}^{c} \lambda_{c d} q_{B}^{d}\right)-\left(\bar{q}_{C}^{a} \lambda_{a b} q_{B}^{b}\right)\left(\bar{q}_{D}^{c} \lambda_{c d} q_{A}^{d}\right), \\
\psi_{7}=\left(\bar{q}_{C}^{a} \gamma_{\mu} \lambda_{a b} q_{A}^{b}\right)\left(\bar{q}_{D}^{c} \gamma^{\mu} \lambda_{c d} q_{B}^{d}\right) \\
-\left(\bar{q}_{C}^{a} \gamma_{\mu} \lambda_{a b} q_{B}^{b}\right)\left(\bar{q}_{D}^{c} \gamma^{\mu} \lambda_{c d} q_{A}^{d}\right), \\
\psi_{8}=\left(\bar{q}_{C}^{a} \sigma_{\mu \nu} \lambda_{a b} q_{A}^{b}\right)\left(\bar{q}_{D}^{c} \sigma^{\mu \nu} \lambda_{c d} q_{B}^{d}\right) \\
-\left(\bar{q}_{C}^{a} \sigma_{\mu \nu} \lambda_{a b} q_{B}^{b}\right)\left(\bar{q}_{D}^{c} \sigma^{\mu \nu} \lambda_{c d} q_{A}^{d}\right), \\
\psi_{9}=\left(\bar{q}_{C}^{a} \gamma_{\mu} \gamma_{5} \lambda_{a b} q_{A}^{b}\right)\left(\bar{q}_{D}^{c} \gamma^{\mu} \gamma_{5} \lambda_{c d} q_{B}^{d}\right) \\
-\left(\bar{q}_{C}^{a} \gamma_{\mu} \gamma_{5} \lambda_{a b} q_{B}^{b}\right)\left(\bar{q}_{D}^{c} \gamma^{\mu} \gamma_{5} \lambda_{c d} q_{A}^{d}\right), \\
\psi_{10}=\left(\bar{q}_{C}^{a} \gamma_{5} \lambda_{a b} q_{A}^{b}\right)\left(\bar{q}_{D}^{c} \gamma_{5} \lambda_{c d} q_{B}^{d}\right) \\
-\left(\bar{q}_{C}^{a} \gamma_{5} \lambda_{a b} q_{B}^{b}\right)\left(\bar{q}_{D}^{c} \gamma_{5} \lambda_{c d} q_{A}^{d}\right) . \\
\end{gathered}
$$

Among these ten currents only five are independent. We can verify the following relations:

$$
\begin{gathered}
\eta_{1}=-\frac{1}{4} \psi_{1}-\frac{1}{4} \psi_{2}+\frac{1}{8} \psi_{3}-\frac{1}{4} \psi_{4}-\frac{1}{4} \psi_{5}, \\
\eta_{2}=\psi_{1}-\frac{1}{2} \psi_{2}+\frac{1}{2} \psi_{4}-\psi_{5}, \\
\eta_{3}=3 \psi_{1}+\frac{1}{2} \psi_{3}+3 \psi_{5}, \\
\eta_{4}=\psi_{1}+\frac{1}{2} \psi_{2}-\frac{1}{2} \psi_{4}-\psi_{5}, \\
\eta_{5}=-\frac{1}{4} \psi_{1}+\frac{1}{4} \psi_{2}+\frac{1}{8} \psi_{3}+\frac{1}{4} \psi_{4}-\frac{1}{4} \psi_{5}, \\
\psi_{6}=-\frac{1}{6} \psi_{1}+\frac{1}{2} \psi_{2}+\frac{1}{4} \psi_{3}-\frac{1}{2} \psi_{4}+\frac{1}{2} \psi_{5}, \\
\psi_{7}=2 \psi_{1}-\frac{5}{3} \psi_{2}-\psi_{4}-2 \psi_{5}, \\
\psi_{8}=6 \psi_{1}-\frac{5}{3} \psi_{3}+6 \psi_{5},
\end{gathered}
$$

$$
\begin{gathered}
\psi_{9}=-2 \psi_{1}-\psi_{2}-\frac{5}{3} \psi_{4}+2 \psi_{5}, \\
\psi_{10}=\frac{1}{2} \psi_{1}-\frac{1}{2} \psi_{2}+\frac{1}{4} \psi_{3}+\frac{1}{2} \psi_{4}-\frac{1}{6} \psi_{5} .
\end{gathered}
$$

D.1.2. Flavor Structure $\mathbf{6}_{f}(q q) \otimes \overline{\mathbf{6}}_{f}(\overline{q q})$. In this subsection we study the scalar tetraquark currents which contain diquarks and antidiquarks having both the symmetric flavor structure $\mathbf{6} \otimes \overline{\mathbf{6}}$. There are altogether five independent scalar tetraquark currents constructed using diquarks and antidiquarks:

$$
\begin{gathered}
\eta_{1}=q_{A}^{a T} \mathbb{C} \gamma_{5} q_{B}^{b}\left(\bar{q}_{C}^{a} \gamma_{5} \mathbb{C} \bar{q}_{D}^{b T}+\bar{q}_{C}^{b} \gamma_{5} \mathbb{C} \bar{q}_{D}^{a T}\right), \\
\eta_{2}=q_{A}^{a T} \mathbb{C} \gamma_{\mu} \gamma_{5} q_{B}^{b}\left(\bar{q}_{C}^{a} \gamma^{\mu} \gamma_{5} \mathbb{C} \bar{q}_{D}^{b T}+\bar{q}_{C}^{b} \gamma^{\mu} \gamma_{5} \mathbb{C} \bar{q}_{D}^{a T}\right), \\
\eta_{3}=q_{A}^{a T} \mathbb{C} \sigma_{\mu \nu} q_{B}^{b}\left(\bar{q}_{C}^{a} \sigma^{\mu \nu} \mathbb{C} \bar{q}_{D}^{b T}-\bar{q}_{C}^{b} \sigma^{\mu \nu} \mathbb{C} \bar{q}_{D}^{a T}\right), \\
\eta_{4}=q_{A}^{a T} \mathbb{C} \gamma_{\mu} q_{B}^{b}\left(\bar{q}_{C}^{a} \gamma^{\mu} \mathbb{C} \bar{q}_{D}^{b T}-\bar{q}_{C}^{b} \gamma^{\mu} \mathbb{C} \bar{q}_{D}^{a T}\right), \\
\eta_{5}=q_{A}^{a T} \mathbb{C} q_{B}^{b}\left(\bar{q}_{C}^{a} \mathbb{C} \bar{q}_{D}^{b T}+\bar{q}_{C}^{b} \mathbb{C} \bar{q}_{D}^{a T}\right) .
\end{gathered}
$$

There are altogether ten scalar tetraquark currents constructed using quark-antiquark pairs:

$$
\begin{gathered}
\psi_{1}=\left(\bar{q}_{C}^{a} q_{A}^{a}\right)\left(\bar{q}_{D}^{b} q_{B}^{b}\right)+\left(\bar{q}_{C}^{a} q_{B}^{a}\right)\left(\bar{q}_{D}^{b} q_{A}^{b}\right), \\
\psi_{2}=\left(\bar{q}_{C}^{a} \gamma_{\mu} q_{A}^{a}\right)\left(\bar{q}_{D}^{b} \gamma^{\mu} q_{B}^{b}\right)+\left(\bar{q}_{C}^{a} \gamma_{\mu} q_{B}^{a}\right)\left(\bar{q}_{D}^{b} \gamma^{\mu} q_{A}^{b}\right), \\
\psi_{3}=\left(\bar{q}_{C}^{a} \sigma_{\mu \nu} q_{A}^{a}\right)\left(\bar{q}_{D}^{b} \sigma^{\mu \nu} q_{B}^{b}\right)+\left(\bar{q}_{C}^{a} \sigma_{\mu \nu} q_{B}^{a}\right)\left(\bar{q}_{D}^{b} \sigma^{\mu \nu} q_{A}^{b}\right), \\
\psi_{4}=\left(\bar{q}_{C}^{a} \gamma_{\mu} \gamma_{5} q_{A}^{a}\right)\left(\bar{q}_{D}^{b} \gamma^{\mu} \gamma_{5} q_{B}^{b}\right)+\left(\bar{q}_{C}^{a} \gamma_{\mu} \gamma_{5} q_{B}^{a}\right)\left(\bar{q}_{D}^{b} \gamma^{\mu} \gamma_{5} q_{A}^{b}\right), \\
\psi_{5}=\left(\bar{q}_{C}^{a} \gamma_{5} q_{A}^{a}\right)\left(\bar{q}_{D}^{b} \gamma_{5} q_{B}^{b}\right)+\left(\bar{q}_{C}^{a} \gamma_{5} q_{B}^{a}\right)\left(\bar{q}_{D}^{b} \gamma_{5} q_{A}^{b}\right), \\
\psi_{6}=\left(\bar{q}_{C}^{a} \lambda_{a b} q_{A}^{b}\right)\left(\bar{q}_{D}^{c} \lambda_{c d} q_{B}^{d}\right)+\left(\bar{q}_{C}^{a} \lambda_{a b} q_{B}^{b}\right)\left(\bar{q}_{D}^{c} \lambda_{c d} q_{A}^{d}\right), \\
\psi_{7}=\left(\bar{q}_{C}^{a} \gamma_{\mu} \lambda_{a b} q_{A}^{b}\right)\left(\bar{q}_{D}^{c} \gamma^{\mu} \lambda_{c d} q_{B}^{d}\right) \\
+\left(\bar{q}_{C}^{a} \gamma_{\mu} \lambda_{a b} q_{B}^{b}\right)\left(\bar{q}_{D}^{c} \gamma^{\mu} \lambda_{c d} q_{A}^{d}\right), \\
\psi_{8}=\left(\bar{q}_{C}^{a} \sigma_{\mu \nu} \lambda_{a b} q_{A}^{b}\right)\left(\bar{q}_{D}^{c} \sigma^{\mu \nu} \lambda_{c d} q_{B}^{d}\right) \\
+\left(\bar{q}_{C}^{a} \sigma_{\mu \nu} \lambda_{a b} q_{B}^{b}\right)\left(\bar{q}_{D}^{c} \sigma^{\mu \nu} \lambda_{c d} q_{A}^{d}\right), \\
\psi_{9}=\left(\bar{q}_{C}^{a} \gamma_{\mu} \gamma_{5} \lambda_{a b} q_{A}^{b}\right)\left(\bar{q}_{D}^{c} \gamma^{\mu} \gamma_{5} \lambda_{c d} q_{B}^{d}\right) \\
+\left(\bar{q}_{C}^{a} \gamma_{\mu} \gamma_{5} \lambda_{a b} q_{B}^{b}\right)\left(\bar{q}_{D}^{c} \gamma^{\mu} \gamma_{5} \lambda_{c d} q_{A}^{d}\right), \\
\psi_{10}=\left(\bar{q}_{C}^{a} \gamma_{5} \lambda_{a b} q_{A}^{b}\right)\left(\bar{q}_{D}^{c} \gamma_{5} \lambda_{c d} q_{B}^{d}\right) \\
+\left(\bar{q}_{C}^{a} \gamma_{5} \lambda_{a b} q_{B}^{b}\right)\left(\bar{q}_{D}^{c} \gamma_{5} \lambda_{c d} q_{A}^{d}\right) . \\
\end{gathered}
$$


Among these ten currents only five are independent. We can verify the following relations:

$$
\begin{gathered}
\eta_{1}=-\frac{1}{4} \psi_{1}-\frac{1}{4} \psi_{2}+\frac{1}{8} \psi_{3}-\frac{1}{4} \psi_{4}-\frac{1}{4} \psi_{5}, \\
\eta_{2}=\psi_{1}-\frac{1}{2} \psi_{2}+\frac{1}{2} \psi_{4}-\psi_{5} \\
\eta_{3}=3 \psi_{1}+\frac{1}{2} \psi_{3}+3 \psi_{5} \\
\eta_{4}=\psi_{1}+\frac{1}{2} \psi_{2}-\frac{1}{2} \psi_{4}-\psi_{5}, \\
\eta_{5}=-\frac{1}{4} \psi_{1}+\frac{1}{4} \psi_{2}+\frac{1}{8} \psi_{3}+\frac{1}{4} \psi_{4}-\frac{1}{4} \psi_{5}, \\
\psi_{6}=-\frac{7}{6} \psi_{1}-\frac{1}{2} \psi_{2}-\frac{1}{4} \psi_{3}+\frac{1}{2} \psi_{4}-\frac{1}{2} \psi_{5}, \\
\psi_{7}=-2 \psi_{1}+\frac{1}{3} \psi_{2}+\psi_{4}+2 \psi_{5}, \\
\psi_{8}=-6 \psi_{1}+\frac{1}{3} \psi_{3}-6 \psi_{5}, \\
\psi_{10}=-\frac{1}{2} \psi_{1}+\frac{1}{2} \psi_{2}-\frac{1}{4} \psi_{3}-\frac{1}{2} \psi_{4}-\frac{7}{6} \psi_{5} . \\
\psi_{2}+\frac{1}{3} \psi_{4}-2 \psi_{5}, \\
\psi_{10}
\end{gathered}
$$

\section{D.2. Pseudoscalar Tetraquark Currents}

D.2.1. Flavor Structure $\overline{\mathbf{3}}_{f}(q q) \otimes \mathbf{3}_{f}(\bar{q} \bar{q})$. In this subsection we study pseudoscalar tetraquark currents which contain diquarks and antidiquarks having both the antisymmetric flavor structure $\overline{\mathbf{3}} \otimes \mathbf{3}$. There are altogether three independent pseudoscalar tetraquark currents constructed using diquarks and antidiquarks:

$$
\begin{gathered}
\eta_{1}=q_{A}^{a T} \mathbb{C} q_{B}^{b}\left(\bar{q}_{C}^{a} \gamma_{5} \mathbb{C} \bar{q}_{D}^{b T}-\bar{q}_{C}^{b} \gamma_{5} C \bar{q}_{D}^{a T}\right), \\
\eta_{2}=q_{A}^{a T} C \gamma_{5} q_{B}^{b}\left(\bar{q}_{C}^{a} \mathbb{C} \bar{q}_{D}^{b T}-\bar{q}_{C}^{b} \mathbb{C} \bar{q}_{D}^{a T}\right), \\
\eta_{3}=q_{A}^{a T} \mathbb{C} \sigma_{\mu \nu} q_{B}^{b}\left(\bar{q}_{C}^{a} \sigma^{\mu \nu} \gamma_{5} \mathbb{C} \bar{q}_{D}^{b T}+\bar{q}_{C}^{b} \sigma^{\mu \nu} \gamma_{5} \mathbb{C} \bar{q}_{D}^{a T}\right) .
\end{gathered}
$$

There are altogether six pseudoscalar tetraquark currents constructed using quark-antiquark pairs:

$$
\begin{gathered}
\psi_{1}=\left(\bar{q}_{C}^{a} q_{A}^{a}\right)\left(\bar{q}_{D}^{b} \gamma_{5} q_{B}^{b}\right)+\left(\bar{q}_{C}^{a} \gamma_{5} q_{A}^{a}\right)\left(\bar{q}_{D}^{b} q_{B}^{b}\right) \\
-\left(\bar{q}_{C}^{a} q_{B}^{a}\right)\left(\bar{q}_{D}^{b} \gamma_{5} q_{A}^{b}\right)-\left(\bar{q}_{C}^{a} \gamma_{5} q_{B}^{a}\right)\left(\bar{q}_{D}^{b} q_{A}^{b}\right), \\
\psi_{2}=\left(\bar{q}_{C}^{a} \gamma_{\mu} q_{A}^{a}\right)\left(\bar{q}_{D}^{b} \gamma^{\mu} \gamma_{5} q_{B}^{b}\right)+\left(\bar{q}_{C}^{a} \gamma_{\mu} \gamma_{5} q_{A}^{a}\right)\left(\bar{q}_{D}^{b} \gamma^{\mu} q_{B}^{b}\right) \\
-\left(\bar{q}_{C}^{a} \gamma_{\mu} q_{B}^{a}\right)\left(\bar{q}_{D}^{b} \gamma^{\mu} \gamma_{5} q_{A}^{b}\right)-\left(\bar{q}_{C}^{a} \gamma_{\mu} \gamma_{5} q_{B}^{a}\right)\left(\bar{q}_{D}^{b} \gamma^{\mu} q_{A}^{b}\right), \\
\psi_{3}=\left(\bar{q}_{C}^{a} \sigma_{\mu \nu} q_{A}^{a}\right)\left(\bar{q}_{D}^{b} \sigma^{\mu \nu} \gamma_{5} q_{B}^{b}\right)-\left(\bar{q}_{C}^{a} \sigma_{\mu \nu} q_{B}^{a}\right)\left(\bar{q}_{D}^{b} \sigma^{\mu \nu} \gamma_{5} q_{A}^{b}\right), \\
\psi_{4}=\lambda_{a b} \lambda_{c d}\left\{\left(\bar{q}_{C}^{a} q_{A}^{b}\right)\left(\bar{q}_{D}^{c} \gamma_{5} q_{B}^{d}\right)+\left(\bar{q}_{C}^{a} \gamma_{5} q_{A}^{b}\right)\left(\bar{q}_{D}^{c} q_{B}^{d}\right)\right. \\
\left.-\left(\bar{q}_{C}^{a} q_{B}^{b}\right)\left(\bar{q}_{D}^{c} \gamma_{5} q_{A}^{d}\right)-\left(\bar{q}_{C}^{a} \gamma_{5} q_{B}^{b}\right)\left(\bar{q}_{D}^{c} q_{A}^{d}\right)\right\},
\end{gathered}
$$

$$
\begin{aligned}
& \psi_{5}=\lambda_{a b} \lambda_{c d}\{\left(\bar{q}_{C}^{a} \gamma_{\mu} q_{A}^{b}\right)\left(\bar{q}_{D}^{c} \gamma^{\mu} \gamma_{5} q_{B}^{d}\right) \\
&+\left(\bar{q}_{C}^{a} \gamma_{\mu} \gamma_{5} q_{A}^{b}\right)\left(\bar{q}_{D}^{c} \gamma^{\mu} q_{B}^{d}\right) \\
&-\left(\bar{q}_{C}^{a} \gamma_{\mu} q_{B}^{b}\right)\left(\bar{q}_{D}^{c} \gamma^{\mu} \gamma_{5} q_{A}^{d}\right) \\
&\left.-\left(\bar{q}_{C}^{a} \gamma_{\mu} \gamma_{5} q_{B}^{b}\right)\left(\bar{q}_{D}^{c} \gamma^{\mu} q_{A}^{d}\right)\right\}, \\
& \psi_{6}=\lambda_{a b} \lambda_{c d}\left\{\left(\bar{q}_{C}^{a} \sigma_{\mu \nu} q_{A}^{b}\right)\left(\bar{q}_{D}^{c} \sigma^{\mu \nu} \gamma_{5} q_{B}^{d}\right)\right. \\
&\left.-\left(\bar{q}_{C}^{a} \sigma_{\mu \nu} q_{B}^{b}\right)\left(\bar{q}_{D}^{c} \sigma^{\mu \nu} \gamma_{5} q_{A}^{d}\right)\right\} .
\end{aligned}
$$

Among these six currents only three are independent. We can verify the following relations:

$$
\begin{gathered}
\eta_{1}=-\frac{1}{4} \psi_{1}-\frac{1}{4} \psi_{2}+\frac{1}{8} \psi_{3}, \\
\eta_{2}=-\frac{1}{4} \psi_{1}+\frac{1}{4} \psi_{2}+\frac{1}{8} \psi_{3}, \\
\eta_{3}=3 \psi_{1}+\frac{1}{2} \psi_{3}, \\
\psi_{4}=\frac{1}{3} \psi_{1}+\frac{1}{2} \psi_{3}, \\
\psi_{5}=-\frac{8}{3} \psi_{2}, \\
\psi_{6}=6 \psi_{1}-\frac{5}{3} \psi_{3} .
\end{gathered}
$$

D.2.2. Flavor Structure $\mathbf{6}_{f}(q q) \otimes \overline{\mathbf{6}}_{f}(\bar{q} \bar{q})$. In this subsection we study pseudoscalar tetraquark currents which contain diquarks and antidiquarks having both the symmetric flavor structure $\mathbf{6} \otimes \overline{\mathbf{6}}$. There are altogether three independent pseudoscalar tetraquark currents constructed using diquarks and antidiquarks:

$$
\begin{gathered}
\eta_{1}=q_{A}^{a T} \mathbb{C} q_{B}^{b}\left(\bar{q}_{C}^{a} \gamma_{5} \mathbb{C} \bar{q}_{D}^{b T}+\bar{q}_{C}^{b} \gamma_{5} \mathbb{C} \bar{q}_{D}^{a T}\right), \\
\eta_{2}=q_{A}^{a T} \mathbb{C} \gamma_{5} q_{B}^{b}\left(\bar{q}_{C}^{a} \mathbb{C} \bar{q}_{D}^{b T}+\bar{q}_{C}^{b} \mathbb{C} \bar{q}_{D}^{a T}\right), \\
\eta_{3}=q_{A}^{a T} \mathbb{C} \sigma_{\mu \nu} q_{B}^{b}\left(\bar{q}_{C}^{a} \sigma^{\mu \nu} \gamma_{5} \mathbb{C} \bar{q}_{D}^{b T}-\bar{q}_{C}^{b} \sigma^{\mu \nu} \gamma_{5} \mathbb{C} \bar{q}_{D}^{a T}\right) .
\end{gathered}
$$

There are altogether six pseudoscalar currents constructed using quark-antiquark pairs:

$$
\begin{aligned}
\psi_{1}= & \left(\bar{q}_{C}^{a} q_{A}^{a}\right)\left(\bar{q}_{D}^{b} \gamma_{5} q_{B}^{b}\right)+\left(\bar{q}_{C}^{a} \gamma_{5} q_{A}^{a}\right)\left(\bar{q}_{D}^{b} q_{B}^{b}\right) \\
& +\left(\bar{q}_{C}^{a} q_{B}^{a}\right)\left(\bar{q}_{D}^{b} \gamma_{5} q_{A}^{b}\right)+\left(\bar{q}_{C}^{a} \gamma_{5} q_{B}^{a}\right)\left(\bar{q}_{D}^{b} q_{A}^{b}\right), \\
\psi_{2}= & \left(\bar{q}_{C}^{a} \gamma_{\mu} q_{A}^{a}\right)\left(\bar{q}_{D}^{b} \gamma^{\mu} \gamma_{5} q_{B}^{b}\right)+\left(\bar{q}_{C}^{a} \gamma_{\mu} \gamma_{5} q_{A}^{a}\right)\left(\bar{q}_{D}^{b} \gamma^{\mu} q_{B}^{b}\right) \\
& +\left(\bar{q}_{C}^{a} \gamma_{\mu} q_{B}^{a}\right)\left(\bar{q}_{D}^{b} \gamma^{\mu} \gamma_{5} q_{A}^{b}\right)+\left(\bar{q}_{C}^{a} \gamma_{\mu} \gamma_{5} q_{B}^{a}\right)\left(\bar{q}_{D}^{b} \gamma^{\mu} q_{A}^{b}\right), \\
\psi_{3}= & \left(\bar{q}_{C}^{a} \sigma_{\mu \nu} q_{A}^{a}\right)\left(\bar{q}_{D}^{b} \sigma^{\mu \nu} \gamma_{5} q_{B}^{b}\right) \\
& +\left(\bar{q}_{C}^{a} \sigma_{\mu \nu} q_{B}^{a}\right)\left(\bar{q}_{D}^{b} \sigma^{\mu \nu} \gamma_{5} q_{A}^{b}\right),
\end{aligned}
$$




$$
\begin{aligned}
& \psi_{4}=\lambda_{a b} \lambda_{c d}\{\left\{\left(\bar{q}_{C}^{a} q_{A}^{b}\right)\left(\bar{q}_{D}^{c} \gamma_{5} q_{B}^{d}\right)\right. \\
&+\left(\bar{q}_{C}^{a} \gamma_{5} q_{A}^{b}\right)\left(\bar{q}_{D}^{c} q_{B}^{d}\right) \\
&+\left(\bar{q}_{C}^{a} q_{B}^{b}\right)\left(\bar{q}_{D}^{c} \gamma_{5} q_{A}^{d}\right) \\
&\left.+\left(\bar{q}_{C}^{a} \gamma_{5} q_{B}^{b}\right)\left(\bar{q}_{D}^{c} q_{A}^{d}\right)\right\}, \\
& \psi_{5}=\lambda_{a b} \lambda_{c d}\left\{\left(\bar{q}_{C}^{a} \gamma_{\mu} q_{A}^{b}\right)\left(\bar{q}_{D}^{c} \gamma^{\mu} \gamma_{5} q_{B}^{d}\right)\right. \\
&+\left(\bar{q}_{C}^{a} \gamma_{\mu} \gamma_{5} q_{A}^{b}\right)\left(\bar{q}_{D}^{c} \gamma^{\mu} q_{B}^{d}\right) \\
&+\left(\bar{q}_{C}^{a} \gamma_{\mu} q_{B}^{b}\right)\left(\bar{q}_{D}^{c} \gamma^{\mu} \gamma_{5} q_{A}^{d}\right) \\
&\left.+\left(\bar{q}_{C}^{a} \gamma_{\mu} \gamma_{5} q_{B}^{b}\right)\left(\bar{q}_{D}^{c} \gamma^{\mu} q_{A}^{d}\right)\right\}, \\
& \psi_{6}=\lambda_{a b} \lambda_{c d}\left\{\left(\bar{q}_{C}^{a} \sigma_{\mu \nu} q_{A}^{b}\right)\left(\bar{q}_{D}^{c} \sigma^{\mu \nu} \gamma_{5} q_{B}^{d}\right)\right. \\
&\left.+\left(\bar{q}_{C}^{a} \sigma_{\mu \nu} q_{B}^{b}\right)\left(\bar{q}_{D}^{c} \sigma^{\mu \nu} \gamma_{5} q_{A}^{d}\right)\right\} .
\end{aligned}
$$

Among these six currents only three are independent. We can verify the following relations:

$$
\begin{gathered}
\eta_{1}=-\frac{1}{4} \psi_{1}-\frac{1}{4} \psi_{2}+\frac{1}{8} \psi_{3}, \\
\eta_{2}=-\frac{1}{4} \psi_{1}+\frac{1}{4} \psi_{2}+\frac{1}{8} \psi_{3}, \\
\eta_{3}=3 \psi_{1}+\frac{1}{2} \psi_{3}, \\
\psi_{4}=-\frac{5}{3} \psi_{1}-\frac{1}{2} \psi_{3}, \\
\psi_{5}=\frac{4}{3} \psi_{2}, \\
\psi_{6}=-6 \psi_{1}+\frac{1}{3} \psi_{3} .
\end{gathered}
$$

D.2.3. Flavor Structure $\overline{\mathbf{3}}_{f}(q q) \otimes \overline{\mathbf{6}}_{f}(\bar{q} \bar{q})$. In this subsection we study pseudoscalar tetraquark currents which contain diquarks having the antisymmetric flavor structure and antidiquarks the symmetric flavor structure $\overline{\mathbf{3}} \otimes \overline{\mathbf{6}}$. There are altogether two independent pseudoscalar tetraquark currents constructed using diquarks and antidiquarks:

$$
\begin{aligned}
\eta_{1} & =q_{A}^{a T} \mathbb{C} \gamma_{\mu} q_{B}^{b}\left(\bar{q}_{C}^{a} \gamma^{\mu} \gamma_{5} \mathbb{C} \bar{q}_{D}^{b T}+\bar{q}_{C}^{b} \gamma^{\mu} \gamma_{5} \mathbb{C} \bar{q}_{D}^{a T}\right), \\
\eta_{2} & =q_{A}^{a T} \mathbb{C} \gamma_{\mu} \gamma_{5} q_{B}^{b}\left(\bar{q}_{C}^{a} \gamma^{\mu} \mathbb{C} \bar{q}_{D}^{b T}-\bar{q}_{C}^{b} \gamma^{\mu} \mathbb{C} \bar{q}_{D}^{a T}\right) .
\end{aligned}
$$

There are altogether four pseudoscalar tetraquark currents constructed using quark-antiquark pairs:

$$
\begin{aligned}
\psi_{1}= & \left(\bar{q}_{C}^{a} q_{A}^{a}\right)\left(\bar{q}_{D}^{b} \gamma_{5} q_{B}^{b}\right)-\left(\bar{q}_{C}^{a} \gamma_{5} q_{A}^{a}\right)\left(\bar{q}_{D}^{b} q_{B}^{b}\right) \\
& -\left(\bar{q}_{C}^{a} q_{B}^{a}\right)\left(\bar{q}_{D}^{b} \gamma_{5} q_{A}^{b}\right)+\left(\bar{q}_{C}^{a} \gamma_{5} q_{B}^{a}\right)\left(\bar{q}_{D}^{b} q_{A}^{b}\right),
\end{aligned}
$$

$$
\begin{aligned}
& \psi_{2}=\left(\bar{q}_{C}^{a} \gamma_{\mu} q_{A}^{a}\right)\left(\bar{q}_{D}^{b} \gamma^{\mu} \gamma_{5} q_{B}^{b}\right)-\left(\bar{q}_{C}^{a} \gamma_{\mu} \gamma_{5} q_{A}^{a}\right)\left(\bar{q}_{D}^{b} \gamma^{\mu} q_{B}^{b}\right) \\
& \text { - }\left(\bar{q}_{C}^{a} \gamma_{\mu} q_{B}^{a}\right)\left(\bar{q}_{D}^{b} \gamma^{\mu} \gamma_{5} q_{A}^{b}\right)+\left(\bar{q}_{C}^{a} \gamma_{\mu} \gamma_{5} q_{B}^{a}\right)\left(\bar{q}_{D}^{b} \gamma^{\mu} q_{A}^{b}\right) \text {, } \\
& \psi_{3}=\lambda_{a b} \lambda_{c d}\left\{\left(\bar{q}_{C}^{a} q_{A}^{b}\right)\left(\bar{q}_{D}^{c} \gamma_{5} q_{B}^{d}\right)-\left(\bar{q}_{C}^{a} \gamma_{5} q_{A}^{b}\right)\left(\bar{q}_{D}^{c} q_{B}^{d}\right)\right. \\
& \left.-\left(\bar{q}_{C}^{a} q_{B}^{b}\right)\left(\bar{q}_{D}^{c} \gamma_{5} q_{A}^{d}\right)+\left(\bar{q}_{C}^{a} \gamma_{5} q_{B}^{b}\right)\left(\bar{q}_{D}^{c} q_{A}^{d}\right)\right\}, \\
& \psi_{4}=\lambda_{a b} \lambda_{c d}\left\{\left(\bar{q}_{C}^{a} \gamma_{\mu} q_{A}^{b}\right)\left(\bar{q}_{D}^{c} \gamma^{\mu} \gamma_{5} q_{B}^{d}\right)\right. \\
& \text { - }\left(\bar{q}_{C}^{a} \gamma_{\mu} \gamma_{5} q_{A}^{b}\right)\left(\bar{q}_{D}^{c} \gamma^{\mu} q_{B}^{d}\right) \\
& \text { - }\left(\bar{q}_{C}^{a} \gamma_{\mu} q_{B}^{b}\right)\left(\bar{q}_{D}^{c} \gamma^{\mu} \gamma_{5} q_{A}^{d}\right) \\
& \left.+\left(\bar{q}_{C}^{a} \gamma_{\mu} \gamma_{5} q_{B}^{b}\right)\left(\bar{q}_{D}^{c} \gamma^{\mu} q_{A}^{d}\right)\right\} .
\end{aligned}
$$

Among these four currents only two are independent. We can verify the following relations:

$$
\begin{gathered}
\eta_{1}=\psi_{1}-\frac{1}{2} \psi_{2}, \\
\eta_{2}=\psi_{1}+\frac{1}{2} \psi_{2}, \\
\psi_{3}=-\frac{2}{3} \psi_{1}-\psi_{2}, \\
\psi_{4}=-4 \psi_{1}-\frac{2}{3} \psi_{2} .
\end{gathered}
$$

\section{Acknowledgments}

The authors would like to thank Professor Shi-Lin Zhu for helpful discussions. This work is partly supported by the National Natural Science Foundation of China under Grant no. 11205011 and the Fundamental Research Funds for the Central Universities.

\section{References}

[1] M. Gell-Mann, "A schematic model of baryons and mesons," Physics Letters, vol. 8, no. 3, pp. 214-215, 1964.

[2] M. Gell-Mann, "Symmetries of baryons and mesons," Physical Review Letters, vol. 125, pp. 1067-1084, 1962.

[3] R. Slansky, "Group theory for unified model building," Physics Reports, vol. 79, no. 1, pp. 1-128, 1981.

[4] J. R. Christman, SU(3) and the Quark Model, University of Michigan, Ann Arbor, Mich, USA, 2001.

[5] J. J. De Swart, "The octet model and its clebsch-gordan coefficients," Reviews of Modern Physics, vol. 35, no. 4, pp. 916-939, 1963, Erratum in, Reviews of Modern Physics, vol. 37, p. 326, 1965.

[6] S. Prelovsek, "Scalar mesons on the lattice," High Energy Physics, vol. 6, no. 1, Article ID 0511110, 2005.

[7] A. H. Fariborz, R. Jora, and J. Schechter, "Global aspects of the scalar meson puzzle," Physical Review D, vol. 79, no. 7, Article ID 074014, 2009.

[8] F. J. Yndurain, R. Garcia-Martin, and J. R. Pelaez, "Experimental status of the $\pi \pi$ isoscalar $S$ wave at low energy: $f_{0}(600)$ pole 
and scattering length," Physical Review, vol. 76, no. 7, Article ID 074034, 10 pages, 2007.

[9] J. Beringer, J. F. Arguin, R. M. Barnett, K. Copic et al., "Review of particle physics," Phyiscal Review D, vol. 86, no. 1, Article ID 010001, 1528 pages, 2012.

[10] R. L. Jaffe, “Exotica," Physics Reports, vol. 409, no. 1, pp. 1-45, 2005.

[11] J. D. Weinstein and N. Isgur, "Do multiquark adrons Exist?" Physical Review Letters, vol. 48, no. 10, pp. 659-662, 1982.

[12] J. D. Weinstein and N. Isgur, " $k \bar{k}$ molecules," Physical Review D, vol. 41, no. 7, pp. 2236-2257, 1990.

[13] S. J. Brodsky and N. Weiss, "Retention of quantum numbers by quark and multiquark jets," Physical Review D, vol. 16, no. 7, pp. 2325-2335, 1977.

[14] A. T. M. Aerts, P. J. Mulders, and J. J. De Swart, "Multiquark states. II. $Q \bar{Q}$ and $Q^{2} \bar{Q}^{2}$ mesons," Physical Review $D$, vol. 21, no. 5, pp. 1370-1387, 1980.

[15] H. J. Lipkin, "A model-independent approach to multiquark bound states," Physical Letters B, vol. 172, pp. 242-247, 1986.

[16] S. Choe, " $\Lambda(1405)$ as a multiquark state," The European Physical Journal A, vol. 3, no. 1, pp. 65-73, 1998.

[17] C. Alexandrou, P. De Forcrand, and A. Tsapalis, "Static threequark SU(3) and four-quark SU(4) potentials," Physical Review D, vol. 65, Article ID 054503, 7 pages, 2002.

[18] F. E. Close and N. A. Tornqvist, "Scalar mesons above and below 1 GeV," Journal of Physics G, vol. 28, no. 10, article R249, 2002.

[19] M. Oka, "Theoretical overview of the pentaquark baryons," Progress of Theoretical Physics, vol. 112, no. 1, pp. 1-19, 2004.

[20] G. J. Ding and M. L. Yan, "A candidate for ${ }^{1}--$ strangeonium hybrid," Physics Letters B, vol. 650, no. 5-6, pp. 390-400, 2007.

[21] S. L. Zhu, "New hadron states," International Journal of Modern Physics E, vol. 17, no. 2, p. 283, 2008.

[22] J. Vijande, A. Valcarce, and J. M. Richard, "Stability of multiquarks in a simple string model," Physical Review D, vol. 76, no. 11, Article ID 114013, 5 pages, 2007.

[23] J.-J. Xie, A. Martinez Torres, E. Oset, and P. Gonzalez, "Plausible explanation for the $\Delta_{5 / 2}+(2000)$ puzzle," Physical Review $C$, vol. 83, no. 5, Article ID 055204, 11 pages, 2011.

[24] A. Martinez Torres, K. P. Khemchandani, L. S. Geng, M. Napsuciale, and E. Oset, " $X(2175)$ as a resonant state of the $\phi K \bar{K}$ system," Physical Review D, vol. 78, no. 7, Article ID 074031, 2008.

[25] A. Martinez Torres, K. P. Khemchandani, D. Jido, and A. Hosaka, "Theoretical support for the $\pi(1300)$ and the recently claimed $f_{0}(1790)$ as molecular resonances ," Physical Review D, vol. 84, no. 7, Article ID 074027, 2011.

[26] C. W. Xiao, M. Bayar, and E. Oset, " $N D K, \bar{K} D N$, and $N D \bar{D}$ molecules," Physical Review D, vol. 84, no. 3, Article ID 034037, 8 pages, 2011.

[27] S. H. Lee and S. Yasui, "Stable multiquark states with heavy quarks in a diquark model," The European Physical Journal C, vol. 64 , no. 2, pp. 283-295, 2009.

[28] H. J. Lipkin, "New predictions for multiquark hadron masses," Physics Letters B, vol. 580, no. 1-2, pp. 50-53, 2004.

[29] J. Ping, H. Huang, C. Deng, F. Wang, and T. Goldman,

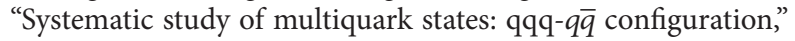
Physical Review C, vol. 79, no. 6, Article ID 065203, 11 pages, 2009.

[30] Z.-F. Sun, X. Liu, M. Nielsen, and S.-L. Zhu, "Hadronic molecules with both open charm and bottom," Physical Review D, vol. 85, no. 9, Article ID 094008, 8 pages, 2012.
[31] R. L. Jaffe, "Multiquark hadrons. I. phenomenology of $Q \bar{Q}$ and $Q^{2} \bar{Q}^{2}$ mesons," Physical Review D, vol. 15, no. 1, pp. 267-280, 1977.

[32] R. L. Jaffe, "Multiquark hadrons. II. methods," Physical Review D, vol. 15, no. 1, pp. 281-289, 1977.

[33] T. Friedmann, "No radial excitations in low energy QCD. I. diquarks and classification of meson," European Physical Journal C, vol. 73, article 2298, 2013.

[34] L. R. Dai, J. Liu, and D. Zhang, "Multiquark states and the mixing of scalar meson," Chinese Physics C, vol. 33, p. 1397, 2009.

[35] A. L. Zhang, "Review on the study of multi-quark states," High Energy Physics and Nuclear Physics, vol. 31, no. 8, pp. 792-796, 2007.

[36] F. Giacosa, "Mixing of scalar tetraquark and quarkonia states in a chiral approach," Physical Review D, vol. 75, no. 5, Article ID 054007, 15 pages, 2007.

[37] A. H. Fariborz, R. Jora, and J. Schechter, "Toy model for two chiral nonets," Physical Review D, vol. 72, no. 3, Article ID 034001, 13 pages, 2005.

[38] M. Napsuciale and S. Rodriguez, "Chiral model for $\bar{q} q$ and $q \bar{q} q q$ mesons," Physical Review D, vol. 70, no. 9, Article ID 094043, 15 pages, 2004.

[39] E. M. Aitala, S. Amato, J. C. Anjos et al., "Experimental evidence for a light and broad scalar resonance in $D^{+} \rightarrow \pi^{-} \pi^{-} \pi^{+}$decay ," Physical Review Letters, vol. 86, no. 5, p. 770, 2001.

[40] M. Ablikim, J.Z. Bai, Y. Ban et al., “The $J / \psi \rightarrow \omega \pi^{+} \pi^{-}$," Physics Letters B, vol. 598, pp. 149-158, 2004.

[41] T. Hatsuda and T. Kunihiro, "QCD phenomenology based on a chiral effective lagrangian," Physics Reports, vol. 247, no. 5-6, pp. 221-367, 1994.

[42] H. X. Chen, A. Hosaka, and S. L. Zhu, "Light scalar tetraquark mesons in the QCD sum rule," Physical Review D, no. 9, Article ID 094025, 15 pages, 2007.

[43] T. V. Brito, F. S. Navarra, M. Nielsen, and M. E. Bracco, "QCD sum rule approach for the light scalar mesons as four-quark states," Physics Letters B, vol. 608, no. 1-2, pp. 69-76, 2005.

[44] S. Weinberg, "Algebraic realizations of chiral symmetry," Physical Review, vol. 177, no. 5, pp. 2604-2620, 1969.

[45] S. Weinberg, "Mended symmetries," Physical Review Letters, vol. 65, no. 10, pp. 1177-1180, 1990.

[46] D. B. Leinweber, "Nucleon properties from unconventional interpolating fields," Physical Review D, vol. 51, no. 11, pp. 63836393, 1995.

[47] D. B. Leinweber, "QCD equalities for baryon current matrix elements," Physical Review D, vol. 53, no. 9, pp. 5115-5124, 1996.

[48] B. L. Ioffe, "Calculation of baryon masses in quantum chromodynamics ," Nuclear Physics B, vol. 188, no. 2, pp. 317-341, Erratum in, Nuclear Physics B, vol. 191, pp. 591-592, 1981.

[49] B. L. Ioffe, "On the choice of quark currents in the QCD sum rules for baryon masses ," Journal of Physics C, vol. 18, no. 1, pp. 67-68, 1983.

[50] Y. Chung, H. G. Dosch, M. Kremer, and D. Schall, "Baryon sum rules and chiral symmetry breaking," Nuclear Physics B, vol. 197, no. 1, pp. 55-75, 1982.

[51] D. Espriu, P. Pascual, and R. Tarrach, "Baryon masses and chiral symmetry breaking," Nuclear Physics B, vol. 214, no. 2, pp. 285$298,1983$.

[52] T. D. Cohen and X. D. Ji, "Chiral multiplets of hadron currents," Physical Review D, vol. 55, no. 11, pp. 6870-6876, 1997. 
[53] H. X. Chen, V. Dmitrasinovic, A. Hosaka, K. Nagata, and S. L. Zhu, "Chiral properties of baryon fields with flavor SU(3) symmetry," Physical Review D, vol. 78, no. 5, Article ID 054021, 2008.

[54] K. Nagata, A. Hosaka, and V. Dmitrasinovic, "Chiral properties of baryon interpolating fields," Modern Physics Letters A, vol. 23, no. 27 n30, p. 2381, 2008.

[55] T. D. Cohen and L. Y. Glozman, "Does one observe chiral symmetry restoration in baryon spectrum?" International Journal of Modern Physics A, vol. 17, no. 10, p. 1327, 2002.

[56] D. Jido, M. Oka, and A. Hosaka, "Chiral symmetry of baryons," Progress of Theoretical Physics, vol. 106, no. 5, pp. 873-908, 2001.

[57] D. Jido, T. Hatsuda, and T. Kunihiro, "Chiral-symmetry realization for even- and odd-parity baryon resonances," Physical Review Letters, vol. 84, no. 15, pp. 3252-3255, 2000.

[58] H.-X. Chen, "The "closed" chiral symmetry and its application to tetraquark," The European Physical Journal C, vol. 72, article 2204, 2012.

[59] S. Narison, QCD as a Theory of Hadrons: From Partons to Confinement, vol. 17 of Cambridge Monographs on Particle Physics, Nuclear Physics and Cosmology, Cambridge University Press, Cambridge, UK, 1st edition, 2002.

[60] C. K. Jiao, W. Chen, H. X. Chen, and S. L. Zhu, "Possible $J^{P C}=0^{--}$ exotic state," Physical Review D, vol. 79, no. 11, Article ID 114034, 10 pages, 2009.

[61] M.-L. Du, W. Chen, X.-L. Chen, and S.-L. Zhu, "Possible $J^{P C}=$ $0^{+-}$exotic states," Chinese Physics C, vol. 37, no. 3, Article ID 033104, 2013.

[62] N. Cardoso, M. Cardoso, and P. Bicudo, "Color fields computed in SU(3) lattice QCD for the static tetraquark system," Physical Review D, vol. 84, no. 5, Article ID 054508, 5 pages, 2011.

[63] F. Okiharu, H. Suganuma, and T. T. Takahashi, "Detailed analysis of the tetraquark potential and flip-flop in SU(3) lattice QCD," Physical Review D, vol. 72, no. 1, Article ID 014505, 17 pages, 2005.

[64] S. Prelovsek, T. Draper, C. B. Lang et al., "Lattice study of light scalar tetraquarks with $I=0,2,1 / 2,3 / 2$ : are $\sigma$ and $\kappa$ tetraquarks?" Physical Review D, vol. 82, no. 9, Article ID 094507, 18 pages, 2010.

[65] M. Loan, Z.-H. Luo, and Y.-Y. Lam, "Lowest-lying tetra-quark hadrons in anisotropic lattice QCD," The European Physical Journal C, vol. 57, no. 3, pp. 579-587, 2008.

[66] S. Prelovsek and D. Mohler, "Lattice study of light scalar tetraquarks," Physical Review D, vol. 79, no. 1, Article ID 014503, 12 pages, 2009.

[67] N. Mathur, A. Alexandru, Y. Chen et al. et al., "Lattice QCD study of the scalar mesons $a_{0}(1450)$ and $\sigma(600)$," Physical Review $D$, vol. 76, no. 11, Article ID 114505, 2007.

[68] C. McNeile and C. Michael, "Properties of light scalar mesons from lattice QCD," Physical Review D, vol. 74, no. 1, Article ID 014508, 9 pages, 2006.

[69] H. X. Chen, A. Hosaka, and S. L. Zhu, "Exotic tetraquark udss of $J^{P}=0^{+}$in the QCD sum rule," Physical Review D, vol. 74, no. 5, Article ID 054001, 11 pages, 2006.

[70] H. X. Chen, X. Liu, A. Hosaka, and S. L. Zhu, "Y(2175) state in the QCD sum rule," Physical Review D, vol. 78, no. 3, Article ID 034012, 8 pages, 2008.

[71] H. X. Chen, A. Hosaka, and S. L. Zhu, " $I^{G} J^{P C}=1^{-} 1^{+}$tetraquark states," Physical Review D, vol. 78, no. 5, Article ID 054017, 14 pages, 2008.
[72] S. Weinberg, "Tetraquark mesons in large- $N$ quantum chromodynamics," Physical Review Letters, vol. 110, Article ID 261601, 2013.

[73] S. Coleman, Aspects of Symmetry, Cambridge University Press, Cambridge, UK, 1985.

[74] P. R. Page, "Large-A/c selection rules for decay of $J_{p c}$ exotichybrid mesons," in Intersections of Particle and Nuclear Physics: 8th Conference, Z. Parsa, Ed., p. 513, American Institute of Physics, College Park, MD, USA, 2003.

[75] M. A. Shifman, A. I. Vainshtein, and V. I. Zakharov, "QCD and resonance physics. theoretical foundations," Nuclear Physics B, vol. 147, no. 5, pp. 385-447, 1979.

[76] L. J. Reinders, H. Rubinstein, and S. Yazaki, "Hadron properties from QCD sum rules," Physics Reports, vol. 127, no. 1, pp. 1-97, 1985.

[77] B. L. Ioffe, V. S. Fadin, V. A. Khoze, and L. N. Lipatov, Quantum Chromodynamics: Perturbative and Nonperturbative Aspects, Cambridge Monographs on Particle Physics, Nuclear Physics and Cosmology, Cambridge University Press, Cambridge, UK, 1st edition, 2010.

[78] A. G. Oganesian, "Small size pentaquark in QCD sum rule approach," High Energy Physics, vol. 1, Article ID 0510327, 2005.

[79] A. G. Oganesian, "Small size pentaquark width: calculation in qcd sum rules," International Journal of Modern Physics A, vol. 22, no. 11, Article ID 051032, pp. 2093-2104, 2005.

[80] K. C. Yang, W. Y. P. Hwang, E. M. Henley, and L. S. Kisslinger, "QCD sum rules and neutron-proton mass difference," Physical Review D, vol. 47, no. 7, pp. 3001-2012, 1993.

[81] W. Y. P. Hwang and K. C. Yang, "QCD sum rules: $\Delta-N$ and $\sum^{0}-\wedge$ mass splittings," Physical Review D, vol. 49, no. 1, pp. 460-465, 1994.

[82] V. Gimenez, V. Lubicz, F. Mescia, V. Porretti, and J. Reyes, "Operator product expansion and quark condensate from lattice QCD in coordinate space," European Physical Journal C, vol. 41, no. 4, pp. 535-544, 2005.

[83] M. Jamin, "Flavour-symmetry breaking of the quark condensate and chiral corrections to the Gell-Mann-Oakes-Renner relation," Physics Letters B, vol. 538, no. 1-2, pp. 71-76, 2002.

[84] B. L. Ioffe and K. N. Zyablyuk, "Gluon condensate in charmonium sum rules with three-loop corrections," European Physical Journal C, vol. 27, no. 2, pp. 229-241, 2003.

[85] A. A. Ovchinnikov and A. A. Pivovarov, "QCD sum rule calculation of the quark gluon condensate," Soviet Journal of Nuclear Physics, vol. 48, p. 721, 1988, Yadernaya Fizika, vol. 48, p. $1135,1988$.

[86] P. Colangelo and A. Khodjamirian, At the Frontier of Particle Physics, Handbook of QCD, vol. 3, World Scientific, Singapore, 2001.

[87] R. D. Matheus, F. S. Navarra, M. Nielsen, and R. Rodrigues da Silva, "Do the QCD sum rules support four-quark states?" Physical Review D, vol. 76, no. 5, Article ID 056005, 7 pages, 2007.

[88] J.-R. Zhang, L.-F. Gan, and M.-Q. Huang, "Search for $Z_{s 1}^{+}$and $Z_{s 2}^{+}$ strangeonium-like structures," Physical Review D, vol. 85 , no. 11, Article ID 116007, 5 pages, 2012.

[89] J.-R. Zhang and G.-F. Chen, "New molecular candidates: $X(1910), X(2200)$, and X(2350)," Physical Review D, vol. 86, no. 11, Article ID 116006, 7 pages, 2012.

[90] V. A. Novikov, M. A. Shifman, A. I. Vaĭnshtĕnn, and V. I. Zakharov, "Wilson's operator expansion: can it fail?" Nuclear Physics B, vol. 249, no. 3, pp. 445-471, 1985, Yadernaya Fizika, vol. 41, p. 1063, 1985. 
[91] B. V. Geshkenbein and B. L. Ioffe, "The role of instantons in generation of mesonic mass spectrum," Nuclear Physics B, vol. 166, no. 2, pp. 340-364, 1980.

[92] T. Schafer, "Instantons and scalar multiquark states: from small to large $N_{c}$," Physical Review D, vol. 68, no. 11, Article ID 114017, 8 pages, 2003.

[93] H.-J. Lee and N. I. Kochelev, "Instanton interpolating current for $\sigma$-tetraquark," Physics Letters B, vol. 642, no. 4, pp. 358-365, 2006. 

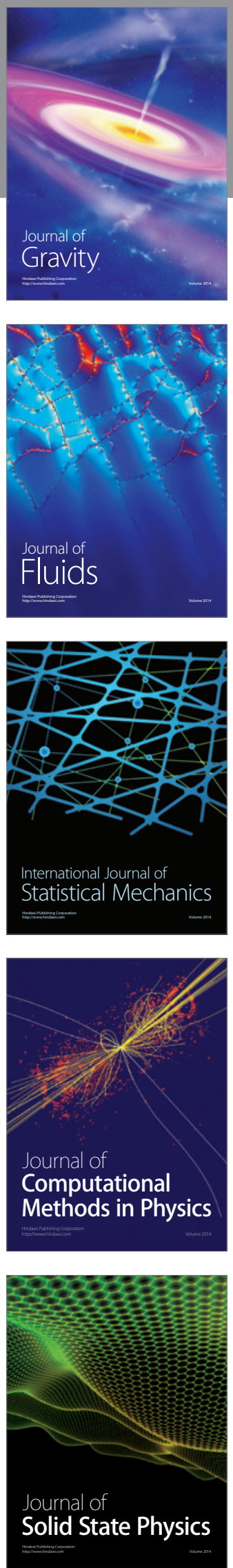

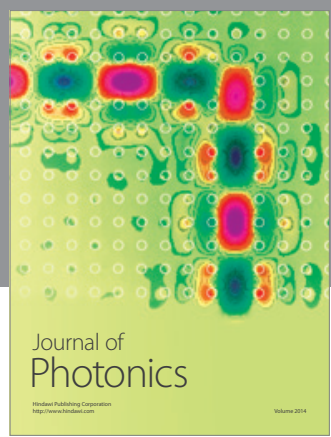

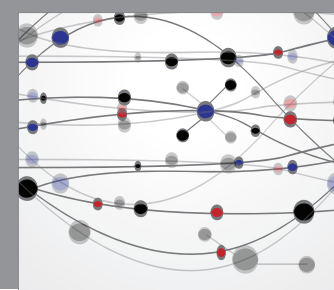

The Scientific World Journal

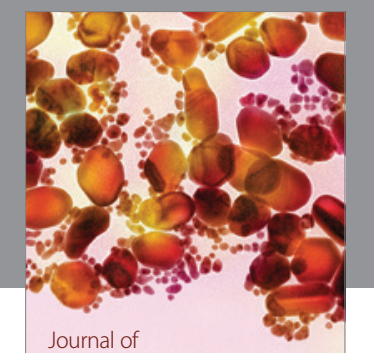

Soft Matter
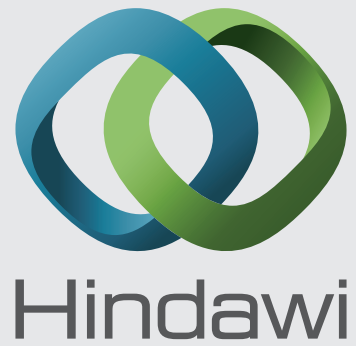

Submit your manuscripts at

http://www.hindawi.com
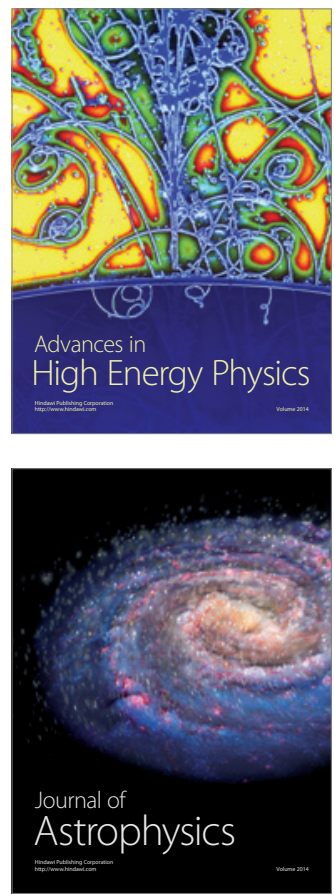
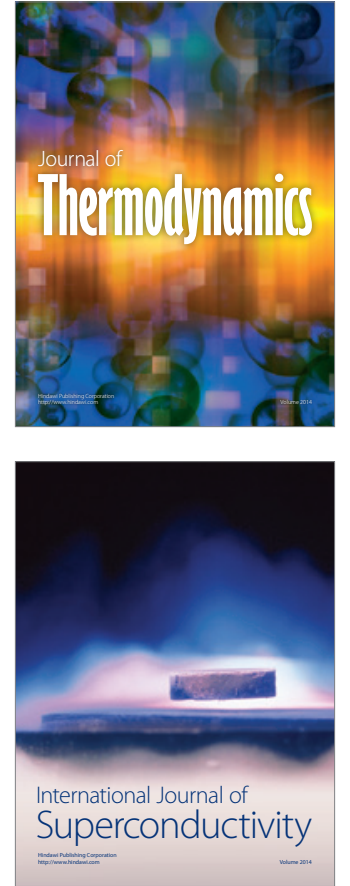
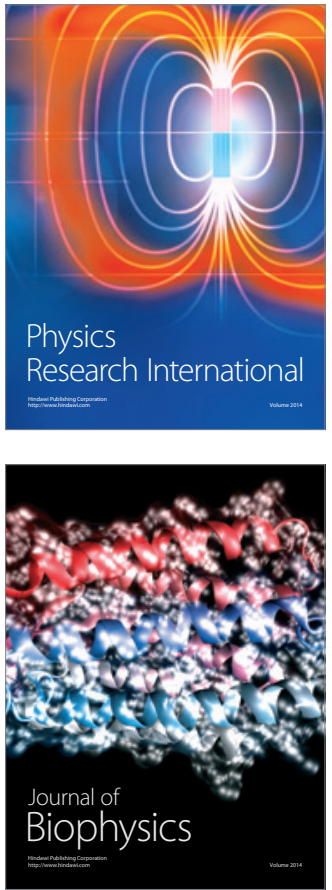
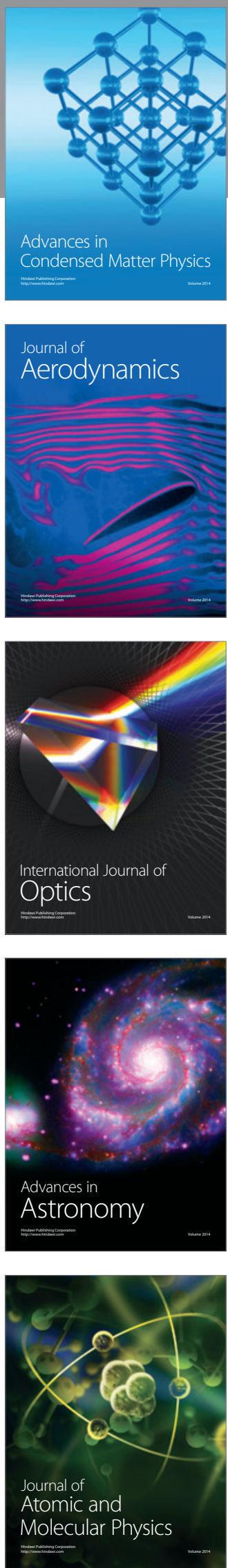